\title{
A Highly Enantioselective Lewis Basic Organocatalyst for Reduction of N-aryl Imines with Exceptional Substrate Spectrum
}

\author{
Zhouyu Wang ${ }^{\dagger, \dagger}$, Xiaoxia Ye ${ }^{\dagger \S}$ Siyu Wei, ${ }^{\dagger}$ Pengcheng, Wu ${ }^{\dagger}$ Anjiang Zhang, ${ }^{\dagger \S}$ Jian Sun $^{\dagger *}$
}

${ }^{\dagger}$ Chengdu Institute of Biology, Chinese Academy of Sciences, Chengdu, 610041 (China)

Fax: (+86) 28-85222753

"Xihua University, Chengdu, 610039 (China)

${ }^{\S}$ Wenzhou University, Wenzhou, 325035 (China)

Graduate School of Chinese Academy of Sciences, China

sunjian@cib.ac.cn

\section{(Supporting Information)}

General: All starting materials were of the highest commercially available grade and used without further purification. All solvents used in the reactions were distilled from appropriate drying agents prior to use. Reactions were monitored by thin layer chromatography using silica gel HSGF254 plates. Flash chromatography was performed using silica gel HG/T2354-92. ${ }^{1} \mathrm{H}-$ and ${ }^{13} \mathrm{C}$ NMR (300 or 600 and 75 or $150 \mathrm{MHz}$, respectively) spectra were recorded in $\mathrm{CDCl}_{3} .{ }^{1} \mathrm{H}$ NMR chemical shifts are reported in ppm $(\boldsymbol{\delta})$ relative to tetramethylsilane (TMS) with the solvent resonance employed as the internal standard $\left(\mathrm{CDCl}_{3}\right.$, $\boldsymbol{\delta} 7.26 \mathrm{ppm}$ ). Data are reported as follows: chemical shift, multiplicity ( $\mathrm{s}=$ singlet, $\mathrm{d}=$ doublet, $\mathrm{q}=$ quartet, $\mathrm{m}=$ multiplet), coupling constants $(\mathrm{Hz})$ and integration. ${ }^{13} \mathrm{C}$ NMR chemical shifts are reported in ppm from tetramethylsilane (TMS) with the solvent resonance as the internal standard $\left(\mathrm{CDCl}_{3}, \boldsymbol{\delta} 77.0\right.$ ppm). ESIMS spectra were recorded on BioTOF Q. HPLC analyses were performed on PerkinElmer (Series 200 UV/VIS Detector and Series 200 Pump). Chiralpak OD-H, AD-H and OJ-H columns were purchased from Daicel Chemical Industries, LTD. All enantiomer ratios have been controlled by coinjections of the pure sample with the racemic substrates. All imines were prepared according to the

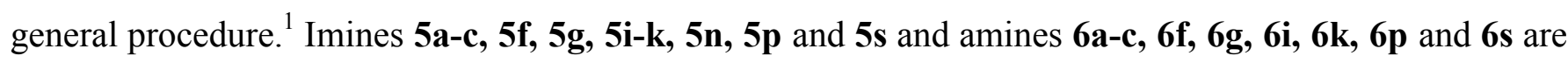
known compounds and their ${ }^{1} \mathrm{H}$ NMR data matched the literature data. ${ }^{1}$ Chemical yields refer to pure isolated substances.

\footnotetext{
${ }^{1}$ (a) Samec, J. S. M.; Bäckvall, J. E. Chem. Eur. J. 2002, 8, 2955. (b) Malkov, A. V.; Mariani, A.; MacDougall, K. N.; Kocovsky, P. Org. Lett. 2004, 6, 2253. (c) Gawinecki, R. Pol. J. Chem. 1987, 61, 589. (d) Barluenga, A.; Aznar, F. Synthesis 1976, 704. (e) Hansen, M. C.; Buchwald, S. L. Org. Lett. 2000, 2, 713. (f) Xiao, D. M.; Zhang, X. M. Angew. Chem..Int. Ed. 2001, 40, 3425. (g) Barluenga, J.; Fernàndez, M. A.; Aznar, F.; Valdès, C. Chem. Eur. J. 2004, 10, 494. (h) Periasamy, M.; Srinivas, G.; Bharath, P. J. org. chem., 1999, 64, 4204. (i) Ozawa, F.; Okamoto, H.; Kawagishi, S.; Yamamoto, S.; Minami, T.; Yoshifuji, M. J. Am. Chem. Soc. 2002, 124, 10968.
} 


\section{Scheme for the synthesis of 4a:}

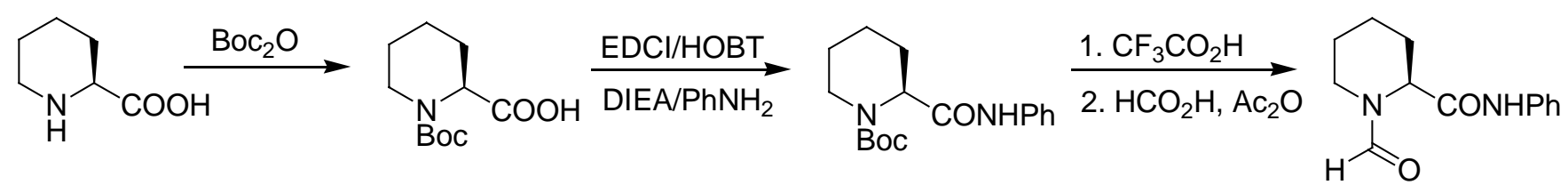

$4 a$

Procedure for the synthesis of 4a: To a solution of L-pipecolinic acid (2.6 g, $20.0 \mathrm{mmol})$ in a mixture of dioxane $(40 \mathrm{~mL})$, water $(20 \mathrm{~mL})$ and $1 \mathrm{~N} \mathrm{NaOH}(20 \mathrm{~mL})$ was added di-tert-butyl pyrocarbonate $(4.8 \mathrm{~g}, 22$ mmol) at $0{ }^{\circ} \mathrm{C}$. The mixture was stirred and warmed to room temperature in $1 \mathrm{~h}$, and then was extracted with ether $(2 \times 40 \mathrm{~mL})$. The aqueous phase was separated and concenteated in vacuo. Ethyl acetate $(50 \mathrm{ml})$ was added and the mixture was cooled down with an ice-water bath. A dilute aqueous solution of $\mathrm{KHSO}_{4}$ was added to adjust the $\mathrm{pH}$ to 2-3. The aqueous layer was separated and extracted with ethyl acetate. The combined extracts was washed with brine, dried over anhydrous $\mathrm{Na}_{2} \mathrm{SO}_{4}$ and evaporated in vacuo. The residue was purified through column chromatography on silica gel (eluent: hexane/EtOAc $=10 / 1)$ to give Boc-L-pipecolinic acid.

To a solution of Boc-L-pipecolinic acid (458 mg, $2.0 \mathrm{mmol})$ in DCM (20 mL) was added aniline (221 uL, $2.4 \mathrm{mmol}$ ), HOBt (350 mg, $2.4 \mathrm{mmol}$ ), N, N-diisopropylethylamine (DIEA, $700 \mu \mathrm{L}$ ) and EDCI (460 $\mathrm{mg}, 2.4 \mathrm{mmol}$ ) at $0{ }^{\circ} \mathrm{C}$. The reaction mixture was stirred at $25^{\circ} \mathrm{C}$ for $12 \mathrm{~h}$, and then concentrated under reduced pressure. The resulting mixture was diluted with EtOAc $(20 \mathrm{~mL})$. The organic layer was separated and washed with saturated aqueous $\mathrm{NaHCO}_{3}(10 \mathrm{~mL})$, aqueous $\mathrm{HCl}(1.0 \mathrm{~N}, 10 \mathrm{~mL})$ and brine $(10 \mathrm{~mL})$, and dried over anhydrous $\mathrm{Na}_{2} \mathrm{SO}_{4}$. After removal of solvents under reduced pressure, the residue was purified through column chromatography on silica gel (eluent: hexane/EtOAc $=10 / 1$ ) to give $(S)$-2phenylcarbamoyl-piperidine-1-carboxylic acid tert-butyl ester.

(S)-2-phenylcarbamoyl-piperidine-1-carboxylic acid tert-butyl ester (559 $\mathrm{mg}, 1.84 \mathrm{mmol}$ ) was dissolved in $20 \mathrm{v} \%$ TFA in $\mathrm{CH}_{2} \mathrm{Cl}_{2}(20 \mathrm{~mL})$ for an hour and then concentrated under reduced pressure. The residue was dissolved in formic acid $(1.5 \mathrm{~mL})$ and the resulting solution was cooled to $0{ }^{\circ} \mathrm{C}$. Acetic anhydride $(1 \mathrm{~mL}, 10.66 \mathrm{mmol})$ was added dropwise and the mixture was allowed to stir at room temperature overnight. After concentration under reduced pressure, the crude product was purified by column chromatography on silica gel (eluent: hexane/EtOAc $=10 / 1)$ to afford pure $4 \mathbf{a}$.

(S)-1-Formyl-piperidine-2-carboxylic acid phenylamide (4a): white solid; yield: $72 \%$; $[\alpha]_{\mathrm{D}}{ }^{20}=-139.2$ $(\mathrm{c}=0.120, \mathrm{EtOH}) ; \mathrm{mp} 129.0-130.0{ }^{\circ} \mathrm{C} ;{ }^{1} \mathrm{H} \mathrm{NMR}\left(600 \mathrm{MHz}, \mathrm{CDCl}_{3}\right): \boldsymbol{\delta}=1.43-1.61(\mathrm{~m}, 2 \mathrm{H}), 1.79(\mathrm{~d}, J$ 
$=11.76 \mathrm{~Hz}, 2 \mathrm{H}), 1.89-1.96(\mathrm{~m}, 1 \mathrm{H}), 2.35(\mathrm{~d}, J=13.86 \mathrm{~Hz}, 1 \mathrm{H}), 3.29(\mathrm{dt}, J=2.7,13.32 \mathrm{~Hz}, 1 \mathrm{H}), 3.60$ $(\mathrm{d}, J=11.94 \mathrm{~Hz}, 1 \mathrm{H}), 5.12(\mathrm{~d}, J=5.94 \mathrm{~Hz}, 1 \mathrm{H}), 7.10(\mathrm{t}, J=7.32 \mathrm{~Hz}, 1 \mathrm{H}), 7.30(\mathrm{t}, J=8.28 \mathrm{~Hz}, 2 \mathrm{H}), 7.50$ $(\mathrm{d}, J=8.28 \mathrm{~Hz}, 2 \mathrm{H}), 8.11$ (brs, $1 \mathrm{H}), 8.18(\mathrm{~s}, 1 \mathrm{H}) ;{ }^{13} \mathrm{C} \mathrm{NMR}\left(150 \mathrm{MHz}, \mathrm{CDCl}_{3}\right.$ ) : $\boldsymbol{\delta}=20.9,24.7,25.6$, 44.6, 51.5, 119.9, 120.2, 124.4, 128.9, 137.7, 163.1, 168.3; ESI HRMS exact mass calcd. for $\left(\mathrm{C}_{13} \mathrm{H}_{16} \mathrm{~N}_{2} \mathrm{O}_{2}\right.$ $+\mathrm{Na})^{+}$requires $\mathrm{m} / \mathrm{z} 255.1104$, found $\mathrm{m} / \mathrm{z} 255.1095$.

\section{Scheme for the synthesis of $4 \mathrm{~b}$ :}

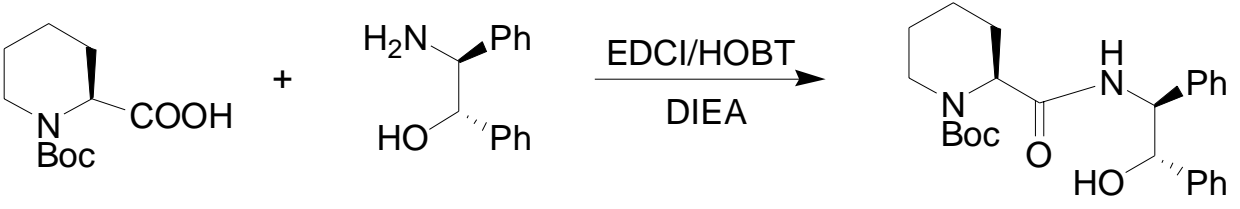

A

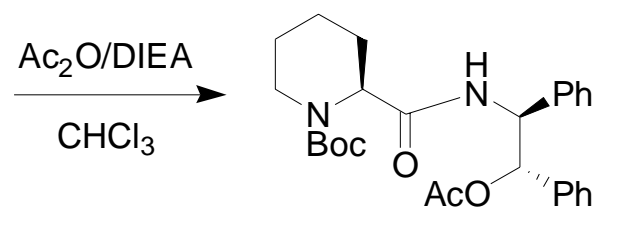

B

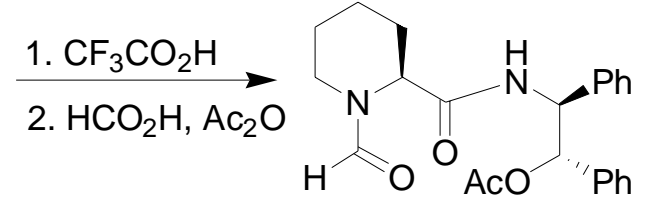

$4 b$

Procedure for the synthesis of $4 \mathbf{b}$ : To a solution of Boc-L-pipecolinic acid (458 mg, $2.0 \mathrm{mmol})$ in DCM (20 mL) was added (1S, 2S)-2-amino-1,2-diphenylehanol (511 mg, $2.4 \mathrm{mmol})$, HOBt (350 mg, $2.4 \mathrm{mmol})$, DIEA $(700 \mu \mathrm{L})$ and EDCI $(460 \mathrm{mg}, 2.4 \mathrm{mmol})$ at $0{ }^{\circ} \mathrm{C}$. The reaction mixture was stirred at $25{ }^{\circ} \mathrm{C}$ for $12 \mathrm{~h}$, and then concentrated under reduced pressure. After the resulting mixture was diluted with EtOAc (20 $\mathrm{mL})$, the organic phase was washed with saturated aqueous $\mathrm{NaHCO}_{3}(10 \mathrm{~mL})$, aqueous $\mathrm{HCl}(1.0 \mathrm{~N}, 10$ $\mathrm{mL})$ and brine $(10 \mathrm{~mL})$, and dried over anhydrous $\mathrm{Na}_{2} \mathrm{SO}_{4}$. After removal of solvents under reduced pressure, the residue was purified through column chromatography on silica gel (eluent: hexane/EtOAc = 5/1) to give A.

To a solution of $\mathbf{A}(763 \mathrm{mg}, 1.80 \mathrm{mmol})$ in $\mathrm{CHCl}_{3}$ was added acetic anhydride $(1 \mathrm{~mL}, 10.66 \mathrm{mmol})$. The mixture was refluxed for $5 \mathrm{~h}$ and then concentrated under reduced pressure. Purification using column chromatography on silica gel (eluent: hexane/EtOAc $=10 / 1)$ gave $\mathbf{B}$.

B $(755 \mathrm{mg}, 1.62 \mathrm{mmol})$ was treated with $20 \mathrm{v} \%$ TFA in $\mathrm{CH}_{2} \mathrm{Cl}_{2}(20 \mathrm{~mL})$ and stirred for $1 \mathrm{~h}$, and then concentrated under reduced pressure. The residue was dissolved in formic acid $(1.5 \mathrm{~mL})$ and the resulting solution was cooled to $0{ }^{\circ} \mathrm{C}$. Acetic anhydride $(1 \mathrm{~mL}, 10.66 \mathrm{mmol})$ was added dropwise and the mixture was allowed to stir at room temperature overnight. After concentration under reduced pressure, the crude product was purified by column chromatography on silica gel (eluent: hexane/EtOAc $=1 / 1$ ) to afford pure $4 \mathbf{b}$. 
(2S,1'S,2'S)-acetic acid 2-[(1-formyl-piperidine-2-carbonyl)-amino]-1,2-diphenyl-ethyl ester (4b): white solid; yield: $68 \%$; $[\alpha]_{\mathrm{D}}{ }^{20}=-28.6(\mathrm{c}=0.168, \mathrm{EtOH}) ; \mathrm{mp} 69-72{ }^{\circ} \mathrm{C} ;{ }^{1} \mathrm{H} \mathrm{NMR}\left(600 \mathrm{MHz}, \mathrm{CDCl}_{3}\right): \boldsymbol{\delta}$ $=1.36-1.58(\mathrm{~m}, 3 \mathrm{H}), 1.66(\mathrm{t}, J=12.90 \mathrm{~Hz}, 2 \mathrm{H}), 2.10(\mathrm{~s}, 3 \mathrm{H}), 2.14(\mathrm{~d}, J=13.32 \mathrm{~Hz}, 1 \mathrm{H}), 3.12(\mathrm{dt}, J=$ 2.88, $12.90 \mathrm{~Hz}, 1 \mathrm{H}), 3.53(\mathrm{~d}, J=9.84 \mathrm{~Hz}, 1 \mathrm{H}), 4.98(\mathrm{~d}, J=5.58 \mathrm{~Hz}, 1 \mathrm{H}), 5.32$ (dd, $J=6.96,8.28 \mathrm{~Hz}$, $1 \mathrm{H}), 6.02(\mathrm{~d}, J=6.54 \mathrm{~Hz}, 1 \mathrm{H}), 7.01-7.28(\mathrm{~m}, 11 \mathrm{H}), 8.17(\mathrm{~s}, 1 \mathrm{H}) ;{ }^{13} \mathrm{C} \mathrm{NMR}\left(150 \mathrm{MHz}, \mathrm{CDCl}_{3}\right): \boldsymbol{\delta}=20$. 9, 21.2, 25.1, 25.5, 26.9, 44.4, 50.9, 58.1, 126.9, 127.0, 127.7, 128.3, 128.5, 128.6, 136.8, 138.5, 162.6, 169.4; ESI HRMS exact mass calcd. for $\left(\mathrm{C}_{23} \mathrm{H}_{26} \mathrm{~N}_{2} \mathrm{O}_{4}+\mathrm{Na}\right)^{+}$requires $\mathrm{m} / \mathrm{z} 417.1785$, found $\mathrm{m} / \mathrm{z} 417.1800$.

\section{The procedures for the syntheses of $4 c$ and $4 d$ are similar to $4 b$}

(2S,1'S,2'R)-acetic acid 2-[(1-formyl-piperidine-2-carbonyl)-amino]-1,2-diphenyl-ethyl ester (4c): white solid; yield: $66 \%$; $[\alpha]_{\mathrm{D}}{ }^{20}=-31.4(\mathrm{c}=0.137, \mathrm{EtOH}), \mathrm{mp} 62-64{ }^{\circ} \mathrm{C} ;{ }^{1} \mathrm{H}$ NMR $\left(600 \mathrm{MHz}, \mathrm{CDCl}_{3}\right): \delta$ $=1.33-1.74(\mathrm{~m}, 5 \mathrm{H}), 2.07(\mathrm{~s}, 3 \mathrm{H}), 2.15(\mathrm{~d}, J=13.62 \mathrm{~Hz}, 1 \mathrm{H}), 3.01(\mathrm{dt}, J=2.70,13.02 \mathrm{~Hz}, 1 \mathrm{H}), 3.43$ $(\mathrm{dd}, J=3.06,13.26 \mathrm{~Hz}, 1 \mathrm{H}), 4.98(\mathrm{~d}, J=5.82 \mathrm{~Hz}, 1 \mathrm{H}), 5.43(\mathrm{dd}, J=5.1 \mathrm{~Hz}, 8.88 \mathrm{~Hz}, 1 \mathrm{H}), 6.07(\mathrm{~d}, J=$ $5.28 \mathrm{~Hz}, 1 \mathrm{H}), 6.84(\mathrm{~m}, 1 \mathrm{H}), 7.01(\mathrm{~m}, 2 \mathrm{H}), 7.07(\mathrm{~m}, 2 \mathrm{H}), 7.11-7.14(\mathrm{~m}, 1 \mathrm{H}), 7.24-7.26(\mathrm{~m}, 4 \mathrm{H}), 7.29(\mathrm{~m}$, $1 \mathrm{H}), 8.12(\mathrm{~s}, 1 \mathrm{H}) ;{ }^{13} \mathrm{C} \mathrm{NMR}\left(150 \mathrm{MHz}, \mathrm{CDCl}_{3}\right): \delta=20.8,21.0,24.9,25.5,26.7,44.3,50.8,56.7,127.1$, 127.4, 127.8, 128.2, 128.3, 128.4, 135.8, 137.4, 162.6, 169.3; ESI HRMS exact mass calcd. for $\left(\mathrm{C}_{23} \mathrm{H}_{26} \mathrm{~N}_{2} \mathrm{O}_{4}+\mathrm{Na}\right)^{+}$requires $\mathrm{m} / \mathrm{z} 417.1785$, found $\mathrm{m} / \mathrm{z} 417.1791$.

(2S,1'R,2'R)-acetic acid 2-[(1-formyl-piperidine-2-carbonyl)-amino]-1,2-diphenyl-ethyl ester (4d): white solid; yield: $69 \%,[\alpha]_{\mathrm{D}}{ }^{20}=-62.1(\mathrm{c}=0.116$, EtOH $), \mathrm{mp} 63-66{ }^{\circ} \mathrm{C} ;{ }^{1} \mathrm{H} \mathrm{NMR}\left(600 \mathrm{MHz}, \mathrm{CDCl}_{3}\right): \delta$ $=1.31-1.89(\mathrm{~m}, 5 \mathrm{H}), 1.99(\mathrm{~s}, 3 \mathrm{H}), 2.16(\mathrm{~d}, J=14.0 \mathrm{~Hz}, 1 \mathrm{H}), 2.65(\mathrm{dt}, J=2.88,13.20 \mathrm{~Hz}, 1 \mathrm{H}), 3.32(\mathrm{~d}, J$ $=13.4 \mathrm{~Hz}, 1 \mathrm{H}), 4.84(\mathrm{~d}, J=5.7 \mathrm{~Hz}, 1 \mathrm{H}), 5.43(\mathrm{dd}, J=6.84,8.94 \mathrm{~Hz}, 1 \mathrm{H}), 6.06(\mathrm{~d}, J=6.72 \mathrm{~Hz}, 1 \mathrm{H})$, $6.88(\mathrm{~d}, J=9.18 \mathrm{~Hz}, 1 \mathrm{H}), 7.10-7.22(\mathrm{~m}, 4 \mathrm{H}), 7.26-7.33(\mathrm{~m}, 6 \mathrm{H}), 7.96(\mathrm{~s}, 1 \mathrm{H}) ;{ }^{13} \mathrm{C} \mathrm{NMR}(150 \mathrm{MHz}$, $\left.\mathrm{CDCl}_{3}\right): \delta=20.8,21.0,24.4,25.4,26.6,44.1,50.6,56.4,127.4,127.6,127.9,128.3,128.4,128.5,136.7$, 137.4, 162.8, 169.0; ESI HRMS exact mass calcd. for $\left(\mathrm{C}_{23} \mathrm{H}_{26} \mathrm{~N}_{2} \mathrm{O}_{4}+\mathrm{Na}\right)^{+}$requires $\mathrm{m} / \mathrm{z} 417.1785$, found $\mathrm{m} / \mathrm{z} 417.1801$.

\section{General procedure for the synthesis of imines 5a-s:}

A mixture of $\mathrm{NaHCO}_{3}(50 \mathrm{mmol})$, amine $(10 \mathrm{mmol})$, ketone $(10 \mathrm{mmol})$ and activated molecular $4 \AA$ sieves $(8.0 \mathrm{~g})$ in anhydrous toluene $(10 \mathrm{~mL})$ was heated at $80{ }^{\circ} \mathrm{C}$ for $12 \mathrm{~h}$ under an argon atmosphere. The 
mixture was filtered through celite. The filtrate was then evaporated in vacuo and the product was crystallized from appropriate solvents or purified by distillation to give pure imine.

5d: Light yellow solid; Yield: $80 \%$; mp $75-76{ }^{\circ} \mathrm{C} ;{ }^{1} \mathrm{H}$ NMR $\left(600 \mathrm{MHz}, \mathrm{CDCl}_{3}\right): \boldsymbol{\delta}=2.21$ (s, 3H), 6.78 $(\mathrm{d}, J=7.74 \mathrm{~Hz}, 2 \mathrm{H}), 7.10(\mathrm{t}, J=7.44 \mathrm{~Hz}, 1 \mathrm{H}), 7.35$ (t, $J=7.44 \mathrm{~Hz}, 2 \mathrm{H}), 7.58(\mathrm{~d}, J=8.28 \mathrm{~Hz}, 2 \mathrm{H}), 7.85$ $(\mathrm{d}, J=8.58 \mathrm{~Hz}, 2 \mathrm{H}) ;{ }^{13} \mathrm{C} \mathrm{NMR}\left(75 \mathrm{MHz}, \mathrm{CDCl}_{3}\right): \boldsymbol{\delta}=17.1,119.2,123.3,125.0,128.7,128.9,131.4$, 138.2, 151.2, 164.2; ESI HRMS exact mass calcd. for $\mathrm{C}_{14} \mathrm{H}_{13} \mathrm{BrN}$ requires $\mathrm{m} / \mathrm{z} 274.0226$, found $\mathrm{m} / \mathrm{z}$ 274.0215 .

5e: Light yellow solid; Yield: $85 \%$; mp $53-55{ }^{\circ} \mathrm{C} ;{ }^{1} \mathrm{H}$ NMR $\left(600 \mathrm{MHz}, \mathrm{CDCl}_{3}\right): \boldsymbol{\delta}=2.20$ (s, 3H), 6.78 $(\mathrm{d}, J=7.32 \mathrm{~Hz}, 2 \mathrm{H}), 7.10(\mathrm{t}, J=7.50 \mathrm{~Hz}, 1 \mathrm{H}), 7.30-7.36(\mathrm{~m}, 3 \mathrm{H}), 7.57-7.59(\mathrm{~m}, 1 \mathrm{H}), 7.87(\mathrm{~d}, J=7.86$ $\mathrm{Hz}, 1 \mathrm{H}), 8.14(\mathrm{~m}, 1 \mathrm{H}) ;{ }^{13} \mathrm{C} \mathrm{NMR}\left(75 \mathrm{MHz}, \mathrm{CDCl}_{3}\right): \boldsymbol{\delta}=17.3,119.2,122.7,123.5,125.7,128.9,129.8$, 130.2, 133.3, 141.4, 151.1, 164.0; ESI HRMS exact mass calcd. for $\mathrm{C}_{14} \mathrm{H}_{13} \mathrm{BrN}$ requires $\mathrm{m} / \mathrm{z} 274.0226$, found $\mathrm{m} / \mathrm{z} 274.0234$.

5h: Light yellow solid; Yield: 82\%; mp $178-181{ }^{\circ} \mathrm{C} ;{ }^{1} \mathrm{H}$ NMR $\left(600 \mathrm{MHz}, \mathrm{CDCl}_{3}\right): \boldsymbol{\delta}=2.32(\mathrm{~s}, 3 \mathrm{H}), 3.93$ (s, 3H), $6.83(\mathrm{~d}, J=8.10 \mathrm{~Hz}, 2 \mathrm{H}), 7.09$ (t, $J=7.32 \mathrm{~Hz}, 1 \mathrm{H}), 7.16-7.18(\mathrm{~m}, 2 \mathrm{H}), 7.35$ (t, $J=7.56 \mathrm{~Hz}, 2 \mathrm{H})$, $7.78(\mathrm{~d}, J=8.64 \mathrm{~Hz}, 1 \mathrm{H}), 7.81(\mathrm{~d}, J=8.82 \mathrm{~Hz}, 1 \mathrm{H}), 8.18(\mathrm{~d}, J=8.64 \mathrm{~Hz}, 1 \mathrm{H}), 8.27(\mathrm{~s}, 1 \mathrm{H}) ;{ }^{13} \mathrm{C} \mathrm{NMR}$ $\left(150 \mathrm{MHz}, \mathrm{CDCl}_{3}\right): \boldsymbol{\delta}=17.3,55.4,105.7,119.2,119.6,123.2,124.8,126.9,127.5,128.3,129.0,130.5$, 134.8, 135.9, 151.9, 158.8, 165.3; ESI HRMS exact mass calcd. for $\mathrm{C}_{19} \mathrm{H}_{18} \mathrm{NO}$ requires m/z 276.1383, found $\mathrm{m} / \mathrm{z} 276.1385$.

5l: Colorless oil; Yield: 60\%; ${ }^{1} \mathrm{H}$ NMR $\left(600 \mathrm{MHz} \mathrm{CDCl}_{3}\right): \boldsymbol{\delta}=2.20(\mathrm{~s}, 3 \mathrm{H}), 6.83(\mathrm{~d}, J=6.72 \mathrm{~Hz}, 1 \mathrm{H})$, $7.03(\mathrm{t}, J=7.44 \mathrm{~Hz}, 1 \mathrm{H}), 7.23-7.25(\mathrm{~m}, 1 \mathrm{H}), 7.41(\mathrm{~d}, J=8.04 \mathrm{~Hz}, 2 \mathrm{H}), 7.45-7.51(\mathrm{~m}, 3 \mathrm{H}), 8.01(\mathrm{~d}, J$ $=6.90 \mathrm{~Hz}, 2 \mathrm{H}) ;{ }^{13} \mathrm{C} \mathrm{NMR}\left(75 \mathrm{MHz}, \mathrm{CDCl}_{3}\right): \boldsymbol{\delta}=18.1,120.6,123.7,124.2,127.4,127.4,128.4,129.8$, 130.9, 138.8, 148.6, 167.7; ESI HRMS exact mass calcd. for $\mathrm{C}_{14} \mathrm{H}_{13} \mathrm{ClN}$ requires $\mathrm{m} / \mathrm{z} 230.0731$, found $\mathrm{m} / \mathrm{z} 230.0742$.

5m: Light yellow solid; Yield: $65 \%$; mp $69-71{ }^{\circ} \mathrm{C} ;{ }^{1} \mathrm{H}$ NMR $\left(600 \mathrm{MHz}, \mathrm{CDCl}_{3}\right): \boldsymbol{\delta}=2.24(\mathrm{~s}, 3 \mathrm{H}), 6.73$ - $6.76(\mathrm{~m}, 2 \mathrm{H}), 7.31-7.33(\mathrm{~m}, 2 \mathrm{H}), 7.44-7.50(\mathrm{~m}, 3 \mathrm{H}), 7.96-7.97(\mathrm{~m}, 2 \mathrm{H}) ;{ }^{13} \mathrm{C} \mathrm{NMR}(75 \mathrm{MHz}$, $\left.\mathrm{CDCl}_{3}\right): \boldsymbol{\delta}=17.3,120.7,122.2,127.1,128.3,128.9,130.6,139.1,150.1,166.1$; ESI HRMS exact mass calcd. for $\mathrm{C}_{14} \mathrm{H}_{13} \mathrm{ClN}$ requires $\mathrm{m} / \mathrm{z} 230.0731$, found $\mathrm{m} / \mathrm{z} 230.0721$. 
5o: Light yellow oil; Yield: 45\%; a 10/1 mixture of $E / Z$ isomers; ${ }^{1} \mathrm{H}$ NMR (600 $\left.\mathrm{MHz}, \mathrm{CDCl}_{3}\right)$ : major isomer: $\boldsymbol{\delta}=1.17-1.70(\mathrm{~m}, 7 \mathrm{H}), 1.73(\mathrm{~s}, 3 \mathrm{H}), 1.82-1.92(\mathrm{~m}, 4 \mathrm{H}), 6.56(\mathrm{~d}, J=8.52 \mathrm{~Hz}, 2 \mathrm{H}), 7.38(\mathrm{~d}, J=$ $8.52 \mathrm{~Hz}, 2 \mathrm{H})$; minor isomer: $\boldsymbol{\delta}=2.07(\mathrm{~s}, 3 \mathrm{H}), 2.25-2.33(\mathrm{~m}, 4 \mathrm{H}) ;{ }^{13} \mathrm{C} \mathrm{NMR}\left(75 \mathrm{MHz}, \mathrm{CDCl}_{3}\right): \boldsymbol{\delta}=17.0$, 25.7, 28.2, 30.0, 49.2, 121.1, 128.66, 131.7, 150.7, 176.3; ESI HRMS exact mass calcd. for $\mathrm{C}_{14} \mathrm{H}_{19} \mathrm{BrN}$ requires $\mathrm{m} / \mathrm{z} 280.0695$, found $\mathrm{m} / \mathrm{z} 280.0698$.

5p: Light yellow oil; Yield: 56\%; a 10/1 mixture of $E / Z$ isomers; ${ }^{1} \mathrm{H}$ NMR $\left(600 \mathrm{MHz}, \mathrm{CDCl}_{3}\right)$ : major isomer: $\boldsymbol{\delta}=1.20-1.72(\mathrm{~m}, 7 \mathrm{H}), 1.73(\mathrm{~s}, 3 \mathrm{H}), 1.82-1.86(\mathrm{~m}, 2 \mathrm{H}), 1.92-1.94(\mathrm{~m}, 2 \mathrm{H}), 6.66-6.67(\mathrm{~m}$, $2 \mathrm{H}), 7.00-7.03(\mathrm{~m}, 1 \mathrm{H}), 7.26-7.27(\mathrm{~m}, 2 \mathrm{H})$; minor isomer: $\boldsymbol{\delta}=2.09(\mathrm{~s}, 3 \mathrm{H}), 2.27-2.32(\mathrm{~m}, 4 \mathrm{H})$; in agreement with the literature data.

5q: Light yellow oil; Yield: 55\%; a 8/1 mixture of $E / Z$ isomers, ${ }^{1} \mathrm{H}$ NMR $\left(300 \mathrm{MHz}, \mathrm{CDCl}_{3}\right): \boldsymbol{\delta}=1.20-$ $1.49(\mathrm{~m}, 7 \mathrm{H}), 1.67(\mathrm{~s}, 3 \mathrm{H}), 1.70-1.96(\mathrm{~m}, 4 \mathrm{H}), 3.74(\mathrm{~s}, 3 \mathrm{H}), 6.62-6.65(\mathrm{~m}, 1 \mathrm{H}), 6.84-6.96(\mathrm{~m}, 2 \mathrm{H})$, $6.97-6.99(\mathrm{~m}, 1 \mathrm{H})$; minor isomer: $\boldsymbol{\delta}=2.11(\mathrm{~s}, 3 \mathrm{H}), 3.76(\mathrm{~s}, 3 \mathrm{H}) ;{ }^{13} \mathrm{C} \mathrm{NMR}\left(75 \mathrm{MHz}, \mathrm{CDCl}_{3}\right): \boldsymbol{\delta}=17.8$, 26.1, 28.4, 30.2, 49.4, 55.6, 111.5, 120.3, 120.8, 123.6, 140.6, 148.9, 177.2; ESI HRMS exact mass calcd. for $\mathrm{C}_{15} \mathrm{H}_{22} \mathrm{NO}$ requires $\mathrm{m} / \mathrm{z} 232.1696$, found $\mathrm{m} / \mathrm{z} 232.1698$.

5r: Light yellow oil; Yield: 46\%; a 6/1 mixture of $E / Z$ isomers; ${ }^{1} \mathrm{H}$ NMR $\left(300 \mathrm{MHz}, \mathrm{CDCl}_{3}\right): \delta=1.20(\mathrm{~d}$, $J=6.88 \mathrm{~Hz}, 6 \mathrm{H}), 1.73(\mathrm{~s}, 3 \mathrm{H}), 2.57-2.67(\mathrm{~m}, 1 \mathrm{H}), 6.66-6.69(\mathrm{~m}, 2 \mathrm{H}), 6.99-7.04(\mathrm{~m}, 1 \mathrm{H}), 7.25-7.31$ (m, 2H); minor isomer: $\boldsymbol{\delta}=1.02(\mathrm{~d}, J=6.88 \mathrm{~Hz}, 6 \mathrm{H}), 2.13(\mathrm{~s}, 3 \mathrm{H}) ;{ }^{13} \mathrm{C} \mathrm{NMR}\left(75 \mathrm{MHz}, \mathrm{CDCl}_{3}\right): \boldsymbol{\delta}=$ 16.9, 19.8, 31.6, 39.0, 119.2, 122.7, 128.7, 151.6, 176.0; ESI HRMS exact mass calcd for $\mathrm{C}_{11} \mathrm{H}_{16} \mathrm{~N}$ requires $\mathrm{m} / \mathrm{z} 162.1277$, found $\mathrm{m} / \mathrm{z} 162.1281$.

5s: Light yellow solid; Yield: 65\%; a 2/1 mixture of $E / Z$ isomers, ${ }^{1} \mathrm{H}$ NMR $\left(300 \mathrm{MHz}, \mathrm{CDCl}_{3}\right): \boldsymbol{\delta}=2.07$ $(\mathrm{s}, 3 \mathrm{H}), 6.68-6.79(\mathrm{~m}, 2 \mathrm{H}), 7.16-7.54(\mathrm{~m}, 10 \mathrm{H})$; minor isomer: $\boldsymbol{\delta}=2.46(\mathrm{~s}, 3 \mathrm{H})$; in agreement with the literature data.

\section{General procedure for the catalytic reduction of imines $5 a-r$.}

Under an argon atmosphere, trichlorosilane ( $40 \mu \mathrm{L}, 0.4 \mathrm{mmol})$ was added dropwise to a stirred solution of imine $5(0.20 \mathrm{mmol})$ and catalyst $4 \mathbf{b}(7.9 \mathrm{mg}, 0.02 \mathrm{mmol})$ in anhydrous $\mathrm{CH}_{2} \mathrm{Cl}_{2}$ at $0{ }^{\circ} \mathrm{C}$. The mixture was allowed to stir at the same temperature for $16 \mathrm{~h}$. The reaction was quenched with a saturated aqueous 
solution of $\mathrm{NaHCO}_{3}(5 \mathrm{~mL})$ and was extracted with EtOAc. The combined extracts was washed with brine and dried over anhydrous $\mathrm{MgSO}_{4}$ and the solvents were evaporated. Purification by column chromatography (silica gel, hexane/EtOAc) afforded pure amine 6 . The ee values were determined using established HPLC techniques with chiral stationary phases.

6a: Light yellow oil; Yield: 97\%, purification by flash chromatography (hexane/EtOAc $=98 / 2) ;{ }^{1} \mathrm{H}$ NMR $\left(300 \mathrm{MHz}, \mathrm{CDCl}_{3}\right): \boldsymbol{\delta}=1.54(\mathrm{~d}, J=6.72 \mathrm{~Hz}, 3 \mathrm{H}), 4.05$ (brs, 1H), $4.51(\mathrm{q}, J=6.72 \mathrm{~Hz}, 1 \mathrm{H}), 6.53(\mathrm{~m}, 2 \mathrm{H})$, $6.66(\mathrm{~m}, 1 \mathrm{H}), 7.08-7.14(\mathrm{~m}, 2 \mathrm{H}), 7.26-7.40(\mathrm{~m}, 5 \mathrm{H})$; in agreement with the literature data.

The enantiomers were analyzed by HPLC using a chiral OD-H column (n-heptane/2-propanol = 99/1, flow rate $=1.0 \mathrm{ml} / \mathrm{min}$, wavelength $=254 \mathrm{~nm}$ ); minor enantiomer: $t_{R}=10.61 \mathrm{~min}$, major enantiomer: $t_{R}=$ $13.99 \mathrm{~min} ; 95 \%$ ee.

6b: Yield : 85\%, purification by flash chromatography (hexane/EtOAc $=98 / 2) ;{ }^{1} \mathrm{H} \mathrm{NMR}(600 \mathrm{MHz}$, $\left.\mathrm{CDCl}_{3}\right): \boldsymbol{\delta}=1.52(\mathrm{~d}, J=6.78 \mathrm{~Hz}, 3 \mathrm{H}), 4.06$ (brs, $\left.1 \mathrm{H}\right), 4.52(\mathrm{q}, J=6.78 \mathrm{~Hz}, 1 \mathrm{H}), 6.47(\mathrm{~d}, J=7.92 \mathrm{~Hz}, 2 \mathrm{H})$, $6.67(\mathrm{t}, J=7.14 \mathrm{~Hz}, 1 \mathrm{H}), 7.10(\mathrm{t}, J=7.68 \mathrm{~Hz}, 2 \mathrm{H}), 7.49(\mathrm{~d}, J=8.1 \mathrm{~Hz}, 2 \mathrm{H}), 7.57(\mathrm{~d}, J=8.1 \mathrm{~Hz}, 2 \mathrm{H})$; in agreement with the literature data.

The enantiomers were analyzed by HPLC using a chiral OD-H column (n-heptane/2-propanol = 90/10, flow rate $=1.0 \mathrm{ml} / \mathrm{min}$, wavelength $=254 \mathrm{~nm}$ ); minor enantiomer: $t_{R}=9.57 \mathrm{~min}$, major enantiomer: $t_{R}=$ $13.82 \mathrm{~min} ; 96 \%$ ee.

6c: Yield: $96 \%$, purification by flash chromatography (hexane/EtOAc $=95 / 5) ;{ }^{1} \mathrm{H}$ NMR $(600 \mathrm{MHz}$, $\left.\mathrm{CDCl}_{3}\right): \delta=1.57(\mathrm{~d}, J=6.72 \mathrm{~Hz}, 3 \mathrm{H}), 4.19(\mathrm{brs}, 1 \mathrm{H}), 4.60(\mathrm{q}, J=6.84 \mathrm{~Hz}, 1 \mathrm{H}), 6.48(\mathrm{~d}, J=7.72 \mathrm{~Hz}, 2 \mathrm{H})$, $6.71(\mathrm{t}, J=7.32 \mathrm{~Hz}, 1 \mathrm{H}), 7.13(\mathrm{t}, J=7.30 \mathrm{~Hz}, 2 \mathrm{H}), 7.56(\mathrm{~d}, J=8.72 \mathrm{~Hz}, 2 \mathrm{H}), 8.19(\mathrm{~d}, J=8.90 \mathrm{~Hz}, 2 \mathrm{H})$; in agreement with the literature data.

The enantiomers were analyzed by HPLC using a chiral OD-H column (n-heptane/2-propanol = 85/15, flow rate $=1.0 \mathrm{ml} / \mathrm{min}$, wavelength $=254 \mathrm{~nm}$ ); minor enantiomer: $t_{R}=19.11 \mathrm{~min}$, major enantiomer: $t_{R}=$ $23.94 \mathrm{~min} ; 95 \%$ ee.

6d: Light yellow solid; Yield: 98\%, purification by flash chromatography (hexane/EtOAc $=98 / 2) ;[\boldsymbol{\alpha}]_{\mathrm{D}}{ }^{20}$ $=-23.4\left(c=0.488\right.$, EtOAc); $\mathrm{mp} 62-65{ }^{\circ} \mathrm{C} ;{ }^{1} \mathrm{H}$ NMR $\left(600 \mathrm{MHz}, \mathrm{CDCl}_{3}\right): \delta=1.48(\mathrm{~d}, J=6.82 \mathrm{~Hz}, 3 \mathrm{H})$, 4.00 (brs, 1H), 4.42 (q, $J=6.84 \mathrm{~Hz}, 1 \mathrm{H}), 6.46$ (d, $J=7.50 \mathrm{~Hz}, 2 \mathrm{H}), 6.65$ (t, $J=7.30 \mathrm{~Hz}, 1 \mathrm{H}), 7.09$ (t, $J=$ $8.52 \mathrm{~Hz}, 2 \mathrm{H}), 7.24(\mathrm{~d}, J=8.3 \mathrm{~Hz}, 2 \mathrm{H}), 7.43(\mathrm{~d}, J=8.32 \mathrm{~Hz}, 2 \mathrm{H}) ;{ }^{13} \mathrm{C} \mathrm{NMR}\left(150 \mathrm{MHz}, \mathrm{CDCl}_{3}\right): \boldsymbol{\delta}=25.1$, 
53.1, 113.3, 117.5, 120.5, 127.7, 129.2, 131.8, 144.4, 147.0; ESI HRMS exact mass calcd. for $\mathrm{C}_{14} \mathrm{H}_{15} \mathrm{BrN}$ requires $\mathrm{m} / \mathrm{z} 276.0382$, found $\mathrm{m} / \mathrm{z} 276.0386$.

The enantiomers were analyzed by HPLC using a chiral OD-H column (n-heptane/2-propanol = 95/5, flow rate $=1.0 \mathrm{ml} / \mathrm{min}$, wavelength $=254 \mathrm{~nm}$ ); minor enantiomer: $t_{R}=10.93$ min, major enantiomer: $t_{R}=$ $14.44 \mathrm{~min} ; 95 \%$ ee.

6e: Light yellow oil; Yield: $82 \%$, purification by flash chromatography (hexane/EtOAc $=98 / 2$ ); $[\alpha]_{\mathrm{D}}{ }^{20}=-$ $17.70\left(c=0.418\right.$, EtOAc), ${ }^{1} \mathrm{H}$ NMR $\left(600 \mathrm{MHz}, \mathrm{CDCl}_{3}\right): \delta=1.50(\mathrm{~d}, J=6.78 \mathrm{~Hz}, 3 \mathrm{H}), 4.02(\mathrm{brs}, 1 \mathrm{H})$, 4.42 (q, $J=6.78 \mathrm{~Hz}, 1 \mathrm{H}), 6.48$ (d, $J=8.46 \mathrm{~Hz}, 2 \mathrm{H}), 6.67$ (t, $J=7.32 \mathrm{~Hz}, 1 \mathrm{H}), 7.10$ (t, $J=8.22 \mathrm{~Hz}$, 2H), $7.18(\mathrm{t}, J=7.86 \mathrm{~Hz}, 1 \mathrm{H}), 7.30(\mathrm{~d}, J=7.50 \mathrm{~Hz}, 1 \mathrm{H}), 7.34-7.36(\mathrm{~m}, 1 \mathrm{H}), 7.52(\mathrm{~s}, 1 \mathrm{H}) ;{ }^{13} \mathrm{C} \mathrm{NMR}(150$ $\left.\mathrm{MHz}, \mathrm{CDCl}_{3}\right): \boldsymbol{\delta}=25.1,53.2,113.3,117.6,122.8,124.5,128.9,129.2,130.0,130.3,146.9,147.9$; ESI HRMS exact mass calcd. for $\mathrm{C}_{14} \mathrm{H}_{15} \mathrm{BrN}$ requires $\mathrm{m} / \mathrm{z} 276.0382$, found $\mathrm{m} / \mathrm{z} 276.0383$.

The enantiomers were analyzed by HPLC using a chiral OD-H column (n-heptane/2-propanol = 95/5, flow rate $=1.0 \mathrm{ml} / \mathrm{min}$, wavelength $=254 \mathrm{~nm}$ ); minor enantiomer: $t_{R}=11.04 \min$, major enantiomer: $t_{R}=$ $15.87 \mathrm{~min} ; 94 \%$ ee.

6f: Yield: 95\%, purification by flash chromatography (hexane/EtOAc $=98 / 2) ;{ }^{1} \mathrm{H}$ NMR $(600 \mathrm{MHz}$, $\left.\mathrm{CDCl}_{3}\right): \boldsymbol{\delta}=1.56(\mathrm{~d}, J=6.72 \mathrm{~Hz}, 3 \mathrm{H}), 3.84(\mathrm{~s}, 3 \mathrm{H}), 4.07$ (brs, $\left.1 \mathrm{H}\right), 4.52$ (q, $\left.J=6.72 \mathrm{~Hz}, 3 \mathrm{H}\right), 6.59$ (d, $J=$ $8.44 \mathrm{~Hz}, 2 \mathrm{H}), 6.72(\mathrm{t}, J=7.32 \mathrm{~Hz}, 1 \mathrm{H}), 6.93(\mathrm{~d}, J=8.64 \mathrm{~Hz}, 2 \mathrm{H}), 7.17(\mathrm{t}, J=8.04 \mathrm{~Hz}, 2 \mathrm{H}), 7.35(\mathrm{~d}, J=$ $8.58 \mathrm{~Hz}, 2 \mathrm{H})$; in agreement with the literature data.

The enantiomers were analyzed by HPLC using a chiral OD-H column (n-heptane/2-propanol = 98/2, flow rate $=1.0 \mathrm{ml} / \mathrm{min}$, wavelength $=254 \mathrm{~nm}$ ); minor enantiomer: $t_{R}=11.75 \mathrm{~min}$, major enantiomer: $t_{R}=$ $14.17 \mathrm{~min} ; 93 \%$ ee.

6g: Yield: 92\%, purification by flash chromatography (hexane/EtOAc $=98 / 2) ;{ }^{1} \mathrm{H} \mathrm{NMR}(600 \mathrm{MHz}$, $\left.\mathrm{CDCl}_{3}\right): \delta=1.65(\mathrm{~d}, J=6.72 \mathrm{~Hz}, 3 \mathrm{H}), 4.19$ (brs, $\left.1 \mathrm{H}\right), 4.71(\mathrm{q}, J=6.54 \mathrm{~Hz}, 1 \mathrm{H}), 6.63(\mathrm{~d}, J=8.32 \mathrm{~Hz}, 2 \mathrm{H})$, $6.72(\mathrm{t}, J=7.14 \mathrm{~Hz}, 1 \mathrm{H}), 7.16(\mathrm{t}, J=8.12 \mathrm{~Hz}, 2 \mathrm{H}), 7.49-7.54(\mathrm{~m}, 2 \mathrm{H}), 7.57(\mathrm{~d}, J=8.46 \mathrm{~Hz}, 1 \mathrm{H}), 7.96-$ $7.89(\mathrm{~m}, 4 \mathrm{H})$; in agreement with the literature data.

The enantiomers were analyzed by HPLC using a chiral OD-H column (n-heptane/2-propanol = 98/2, flow rate $=1.0 \mathrm{ml} / \mathrm{min}$, wavelength $=254 \mathrm{~nm}$ ); minor enantiomer: $t_{R}=14.33 \mathrm{~min}$, major enantiomer: $t_{R}=$ $16.61 \mathrm{~min} ; 93 \%$ ee. 
6h: Yield: $91 \%$, purification by flash chromatography (hexane/EtOAc $=98 / 2) ;[\alpha]_{\mathrm{D}}{ }^{20}=-38.0(c=0.142$, EtOAc); mp $132-133{ }^{\circ} \mathrm{C} ;{ }^{1} \mathrm{H}$ NMR $\left(600 \mathrm{MHz}, \mathrm{CDCl}_{3}\right): \delta=1.57(\mathrm{~d}, J=6.72 \mathrm{~Hz}, 3 \mathrm{H}), 3.89$ (s, 3H), 4.12 (brs, 1H), $4.60(\mathrm{q}, J=6.72 \mathrm{~Hz}, 1 \mathrm{H}), 6.55(\mathrm{~d}, J=8.20 \mathrm{~Hz}, 2 \mathrm{H}), 6.63(\mathrm{t}, J=7.44 \mathrm{~Hz}, 1 \mathrm{H}), 7.06-7.13(\mathrm{~m}$, 4H), $7.46(\mathrm{~d}, J=8.40 \mathrm{~Hz}, 1 \mathrm{H}), 7.67-7.73(\mathrm{~m}, 3 \mathrm{H}) ;{ }^{13} \mathrm{C} \mathrm{NMR}\left(150 \mathrm{MHz}, \mathrm{CDCl}_{3}\right): \boldsymbol{\delta}=25.0,53.7,55.3$, $105.7,113.4,117.3,118.8,124.2$, 124.9, 127.3, 129.0, 129.1, 129.3, 133.8, 140.4, 147.4, 157.5; ESI HRMS exact mass calcd. for $\left(\mathrm{C}_{19} \mathrm{H}_{19} \mathrm{NO}+\mathrm{Na}\right)^{+}$requires m/z 300.1359, found m/z 300.1349.

The enantiomers were analyzed by HPLC using a chiral OD-H column (n-heptane/2-propanol = 98/2, flow rate $=1.0 \mathrm{ml} / \mathrm{min}$, wavelength $=254 \mathrm{~nm}$ ); minor enantiomer: $t_{R}=16.60 \mathrm{~min}$, major enantiomer: $t_{R}=$ $20.91 \mathrm{~min} ; 90 \%$ ee.

6i: Yield: 98\%, purification by flash chromatography (hexane/EtOAc $=98 / 2) ;{ }^{1} \mathrm{H} \mathrm{NMR}(600 \mathrm{MHz}$, $\left.\mathrm{CDCl}_{3}\right): \boldsymbol{\delta}=1.50(\mathrm{~d}, J=6.66 \mathrm{~Hz}, 3 \mathrm{H}), 3.68(\mathrm{~s}, 3 \mathrm{H}), 4.40(\mathrm{q}, J=6.66 \mathrm{~Hz}, 1 \mathrm{H}), 6.47(\mathrm{~d}, J=8.88 \mathrm{~Hz}, 2 \mathrm{H})$, $6.69(\mathrm{~d}, J=8.88 \mathrm{~Hz}, 2 \mathrm{H}), 7.20-7.23(\mathrm{~m}, 1 \mathrm{H}), 7.31(\mathrm{t}, J=7.38 \mathrm{~Hz}, 2 \mathrm{H}), 7.36(\mathrm{~d}, J=7.2 \mathrm{~Hz}, 2 \mathrm{H})$; in agreement with the literature data.

The enantiomers were analyzed by HPLC using a chiral AD-H column (n-heptane/2-propanol = 98/2, flow rate $=1.0 \mathrm{ml} / \mathrm{min}$, wavelength $=254 \mathrm{~nm}$ ); major enantiomer: $t_{R}=10.07$ min, minor enantiomer: $t_{R}=$ $11.12 \mathrm{~min} ; 92 \%$ ee.

6j: Light yellow solid; Yield: $90 \%$, purification by flash chromatography (hexane/EtOAc $=98 / 2) ;[\boldsymbol{\alpha}]_{\mathrm{D}}^{20}$ $=-35.1\left(c=0.646\right.$, EtOAc); mp $62-64{ }^{\circ} \mathrm{C} ;{ }^{1} \mathrm{H}$ NMR $\left(600 \mathrm{MHz}, \mathrm{CDCl}_{3}\right): \boldsymbol{\delta}=1.50(\mathrm{~d}, J=6.66 \mathrm{~Hz}, 3 \mathrm{H})$, 3.90 (brs, 1H), $4.45(\mathrm{q}, J=6.66 \mathrm{~Hz}, 1 \mathrm{H}), 6.43(\mathrm{~d}, J=8.40 \mathrm{~Hz}, 2 \mathrm{H}), 6.43(\mathrm{~d}, J=8.04 \mathrm{~Hz}, 2 \mathrm{H}), 7.20-7.22$ $(\mathrm{m}, 1 \mathrm{H}), 7.31(\mathrm{t}, J=7.62 \mathrm{~Hz}, 2 \mathrm{H}), 7.36(\mathrm{~d}, J=7.26 \mathrm{~Hz}, 2 \mathrm{H}) ;{ }^{13} \mathrm{C} \mathrm{NMR}\left(150 \mathrm{MHz}, \mathrm{CDCl}_{3}\right): \boldsymbol{\delta}=20.4,25.1$, 53.7, 113.4, 125.9, 126.4, 126.8, 128.6, 129.6, 145.0, 145.4; ESI HRMS exact mass calcd. for $\mathrm{C}_{15} \mathrm{H}_{18} \mathrm{~N}$ requires $\mathrm{m} / \mathrm{z} 212.1434$, found $\mathrm{m} / \mathrm{z} 212.1440$.

The enantiomers were analyzed by HPLC using a chiral OD-H column (n-heptane/2-propanol = 99/1, flow rate $=1.0 \mathrm{ml} / \mathrm{min}$, wavelength $=254 \mathrm{~nm}$ ); major enantiomer: $t_{R}=9.59$ min, minor enantiomer: $t_{R}=$ $11.03 \mathrm{~min} ; 95 \%$ ee.

6k: Yield: 92\%, purification by flash chromatography (hexane/EtOAc $=98 / 2)$; ${ }^{1} \mathrm{H}$ NMR $(600 \mathrm{MHz}$, $\left.\mathrm{CDCl}_{3}\right): \boldsymbol{\delta}=1.55(\mathrm{~d}, J=6.66 \mathrm{~Hz}, 3 \mathrm{H}), 3.88(\mathrm{~s}, 3 \mathrm{H}), 4.47(\mathrm{q}, J=6.66 \mathrm{~Hz}, 1 \mathrm{H}), 4.63(\mathrm{brs}, 1 \mathrm{H}), 6.33(\mathrm{~d}, J=$ $7.8 \mathrm{~Hz}, 1 \mathrm{H}), 6.60(\mathrm{t}, J=7.8 \mathrm{~Hz}, 1 \mathrm{H}), 6.69$ (t, $J=7.8 \mathrm{~Hz}, 1 \mathrm{H}), 6.76(\mathrm{~d}, J=7.8 \mathrm{~Hz}, 1 \mathrm{H}), 7.22$ (t, $J=7.3 \mathrm{~Hz}$, $1 \mathrm{H}), 7.31(\mathrm{~d}, J=7.44 \mathrm{~Hz}, 2 \mathrm{H}) ; 7.37(\mathrm{~d}, J=7.8 \mathrm{~Hz}, 2 \mathrm{H})$; in agreement with the literature data. 
The enantiomers were analyzed by HPLC using a chiral OD-H column (n-heptane/2-propanol = 99/1, flow rate $=1.0 \mathrm{ml} / \mathrm{min}$, wavelength $=254 \mathrm{~nm}$ ); minor enantiomer: $t_{R}=6.78 \mathrm{~min}$, major enantiomer: $t_{R}=$ $9.47 \mathrm{~min} ; 89 \%$ ee.

61: Colorless oil; Yield: 93\%, purification by flash chromatography (hexane/EtOAc $=98 / 2) ;[\alpha]_{\mathrm{D}}{ }^{20}=-$ $89.02\left(c=0.1\right.$, EtOAc); ${ }^{1} \mathrm{H}$ NMR $\left(300 \mathrm{MHz}, \mathrm{CDCl}_{3}\right): \delta=1.60(\mathrm{~d}, J=6.71 \mathrm{~Hz}, 3 \mathrm{H}), 4.53-4.57(\mathrm{~m}, 1 \mathrm{H})$, 4.72 (brs, 1H), 6.42 (d, $J=8.20 \mathrm{~Hz}, 1 \mathrm{H}), 6.61$ (t, $J=7.71 \mathrm{~Hz}, 1 \mathrm{H}), 6.98$ (t, $J=7.60 \mathrm{~Hz}, 1 \mathrm{H}), 7.26-7.39$ $(\mathrm{m}, 6 \mathrm{H}) ;{ }^{13} \mathrm{C} \mathrm{NMR}\left(150 \mathrm{MHz}, \mathrm{CDCl}_{3}\right): \boldsymbol{\delta}=25.2,53.4,112.5,117.2,118.9,125.7,127.0,127.6,128.7$, 128.9, 143.0, 144.6; ESI HRMS exact mass calcd. for $\mathrm{C}_{14} \mathrm{H}_{15} \mathrm{ClN}$ requires $\mathrm{m} / \mathrm{z} 232.0888$, found $\mathrm{m} / \mathrm{z}$ 232.0752 .

The enantiomers were analyzed by HPLC using a chiral OD-H column (n-heptane/2-propanol = 99/1, flow rate $=1.0 \mathrm{ml} / \mathrm{min}$, wavelength $=254 \mathrm{~nm}$ ); minor enantiomer: $t_{R}=5.73$ min, major enantiomer: $t_{R}=$ $8.98 \mathrm{~min} ; 90 \%$ ee.

6m: Colorless oil; Yield: $98 \%$, purification by flash chromatography (hexane/EtOAc $=98 / 2) ;[\alpha]_{\mathrm{D}}{ }^{20}=-$ $15.10\left(c=0.682\right.$, EtOAc); ${ }^{1} \mathrm{H}$ NMR $\left(600 \mathrm{MHz}, \mathrm{CDCl}_{3}\right): \boldsymbol{\delta}=1.51(\mathrm{~d}, J=6.73 \mathrm{~Hz}, 3 \mathrm{H}), 4.07$ (brs, $\left.1 \mathrm{H}\right)$, $4.44(\mathrm{q}, J=6.69 \mathrm{~Hz}, 1 \mathrm{H}), 6.42$ (d, $J=8.75 \mathrm{~Hz}, 2 \mathrm{H}), 7.02$ (d, $J=8.73 \mathrm{~Hz}, 2 \mathrm{H}), 7.22$ - 7.24 (m, 1H), 7.30 $7.33(\mathrm{~m}, 4 \mathrm{H}) ;{ }^{13} \mathrm{C} \mathrm{NMR}\left(150 \mathrm{MHz}, \mathrm{CDCl}_{3}\right): \boldsymbol{\delta}=24.9,53.6,114.4,121.8,125.8,127.0,128.7,128.9$, 144.7, 145.8; ESI HRMS exact mass calcd. for $\mathrm{C}_{14} \mathrm{H}_{15} \mathrm{ClN}$ requires $\mathrm{m} / \mathrm{z} 232.0888$, found m/z 232.0877.

The enantiomers were analyzed by HPLC using a chiral OD-H column (n-heptane/2-propanol = 99/1, flow rate $=1.0 \mathrm{ml} / \mathrm{min}$, wavelength $=254 \mathrm{~nm}$ ); major enantiomer: $t_{R}=10.48 \mathrm{~min}$, minor enantiomer: $t_{R}=$ $14.39 \mathrm{~min} ; 93 \%$ ee.

6n: Light yellow solid; Yield: 91\%, purification by flash chromatography (hexane/EtOAc $=98 / 2) ;[\boldsymbol{\alpha}]_{\mathrm{D}}{ }^{20}$ $=-4.2\left(c=0.166\right.$, EtOAc); mp $67-68{ }^{\circ} \mathrm{C} ;{ }^{1} \mathrm{H}$ NMR $\left(600 \mathrm{MHz}, \mathrm{CDCl}_{3}\right): \boldsymbol{\delta}=1.51(\mathrm{~d}, J=6.72 \mathrm{~Hz}, 3 \mathrm{H})$, 4.07 (brs, 1H), 4.43 (q, $J=6.48 \mathrm{~Hz}, 1 \mathrm{H}), 6.36$ - $6.38(\mathrm{~m}, 2 \mathrm{H}), 7.13$ - $7.16(\mathrm{~m}, 2 \mathrm{H}), 7.22$ - $7.25(\mathrm{~m}, 1 \mathrm{H})$, 7.32 - $7.33(\mathrm{~m}, 4 \mathrm{H}) ;{ }^{13} \mathrm{C}$ NMR $\left(150 \mathrm{MHz}, \mathrm{CDCl}_{3}\right): \boldsymbol{\delta}=25.0,53.5,108.9,114.9,125.8,127.1,128.7$, 131.8, 144.6, 146.2; ESI HRMS exact mass calcd. for $\mathrm{C}_{14} \mathrm{H}_{15} \mathrm{BrN}$ requires $\mathrm{m} / \mathrm{z} 276.0382$, found $\mathrm{m} / \mathrm{z}$ 276.0378 .

The enantiomers were analyzed by HPLC using a chiral OD-H column (n-heptane/2-propanol = 99/1, flow rate $=1.0 \mathrm{ml} / \mathrm{min}$, wavelength $=254 \mathrm{~nm}$ ); major enantiomer: $t_{R}=11.35 \mathrm{~min}$, minor enantiomer: $t_{R}=$ $15.39 \mathrm{~min} ; 93 \%$ ee. 
6o: Light yellow oil; Yield: $80 \%$, purification by flash chromatography (hexane/EtOAc $=98 / 2) ;[\alpha]_{\mathrm{D}}{ }^{20}=$ $6.43\left(c=0.140\right.$, EtOAc), ${ }^{1} \mathrm{H}$ NMR $\left(600 \mathrm{MHz}, \mathrm{CDCl}_{3}\right): \delta=0.96-1.08(\mathrm{~m}, 2 \mathrm{H}), 1.10(\mathrm{~d}, J=6.54 \mathrm{~Hz}, 3 \mathrm{H})$, $1.12-1.25(\mathrm{~m}, 3 \mathrm{H}), 1.38-1.44(\mathrm{~m}, 1 \mathrm{H}), 1.65-1.69(\mathrm{~m}, 2 \mathrm{H}), 1.74-1.80(\mathrm{~m}, 3 \mathrm{H}), 3.23-3.26(\mathrm{~m}, 1 \mathrm{H})$, 3.50 (brs, 1H), 6.42 - 6.44 (m, 2H), 7.19 - $7.22(\mathrm{~m}, 2 \mathrm{H}) ;{ }^{13} \mathrm{C} \mathrm{NMR}\left(150 \mathrm{MHz}, \mathrm{CDCl}_{3}\right): \boldsymbol{\delta}=17.3,26.3$, 26.4, 26.6, 28.4, 29.7, 42. 9, 53.2, 107.8, 114.5, 131.9, 146.9; ESI HRMS exact mass calcd. for $\mathrm{C}_{14} \mathrm{H}_{21} \mathrm{BrN}$ requires $\mathrm{m} / \mathrm{z} 282.0852$, found $\mathrm{m} / \mathrm{z} 282.0845$.

The enantiomers were analyzed by HPLC using a chiral OD-H column (n-heptane/2-propanol = 99/1, flow rate $=1.0 \mathrm{ml} / \mathrm{min}$, wavelength $=254 \mathrm{~nm}$ ); major enantiomer: $t_{R}=5.46$ min, minor enantiomer: $t_{R}=$ $6.87 \mathrm{~min} ; 95 \%$ ee.

6p: Yield: $81 \%$, purification by flash chromatography (hexane/EtOAc $=98 / 2) ;{ }^{1} \mathrm{H}$ NMR $(600 \mathrm{MHz}$, $\left.\mathrm{CDCl}_{3}\right): \boldsymbol{\delta}=1.00-1.08(\mathrm{~m}, 2 \mathrm{H}), 1.11(\mathrm{~d}, J=6.42 \mathrm{~Hz}, 3 \mathrm{H}), 1.13-1.28(\mathrm{~m}, 3 \mathrm{H}), 1.41-1.47(\mathrm{~m}, 1 \mathrm{H})$, $1.65-1.83(\mathrm{~m}, 5 \mathrm{H}), 6.56(\mathrm{~d}, J=8.46 \mathrm{~Hz}, 2 \mathrm{H}), 6.62-6.64(\mathrm{~m}, 1 \mathrm{H}), 7.13-7.16(\mathrm{~m}, 2 \mathrm{H})$; in agreement with the literature data.

The enantiomers were analyzed by HPLC using a chiral OJ-H column (n-heptane/2-propanol = 99/1, flow rate $=0.5 \mathrm{ml} / \mathrm{min}$, wavelength $=254 \mathrm{~nm}$ ); major enantiomer: $t_{R}=20.54 \min$, minor enantiomer: $t_{R}=$ $22.96 \mathrm{~min} ; 95 \%$ ee.

6q: Light yellow oil; Yield: 75\%, purification by flash chromatography (hexane/EtOAc $=98 / 2) ;[\alpha]_{\mathrm{D}}{ }^{20}=$ $-23.73\left(c=0.118\right.$, EtOAc), ${ }^{1} \mathrm{H}$ NMR $\left(300 \mathrm{MHz}, \mathrm{CDCl}_{3}\right): \delta=1.07-1.25(\mathrm{~m}, 8 \mathrm{H}), 1.66-2.14(\mathrm{~m}, 6 \mathrm{H})$, $3.28-3.36(\mathrm{~m}, 1 \mathrm{H}), 3.85(\mathrm{~s}, 3 \mathrm{H}), 4.15(\mathrm{brs}, 1 \mathrm{H}), 6.57-6.63(\mathrm{~m}, 2 \mathrm{H}), 6.75-6.77(\mathrm{~m}, 1 \mathrm{H}), 6.82-6.87(\mathrm{~m}$, $1 \mathrm{H}) ;{ }^{13} \mathrm{C}$ NMR $\left(75 \mathrm{MHz}, \mathrm{CDCl}_{3}\right): \boldsymbol{\delta}=17.4,26.4,26.5,26.6,28.4,29.8,42.8,52.6,55.4,109.5,109.8$, 115.3, 121.2, 137.8, 146.6; ESI HRMS exact mass calcd. for $\mathrm{C}_{15} \mathrm{H}_{24} \mathrm{NO}$ requires $\mathrm{m} / \mathrm{z} 234.1852$, found $\mathrm{m} / \mathrm{z}$ 234.1863.

The enantiomers were analyzed by HPLC using a chiral OJ-H column (n-heptane/2-propanol = 99/1, flow rate $=1.0 \mathrm{ml} / \mathrm{min}$, wavelength $=254 \mathrm{~nm}$ ); major enantiomer: $t_{R}=5.43 \mathrm{~min}$, minor enantiomer: $t_{R}=6.39$ $\min ; 87 \%$ ee.

6r: Light yellow oil; Yield: $86 \%$, purification by flash chromatography (2\% EtOAc in Hexanes). $[\alpha]_{\mathrm{D}}{ }^{20}=$ $+49.29(c=0.14$, EtOAC $),{ }^{1} \mathrm{H}$ NMR $\left(300 \mathrm{MHz}, \mathrm{CDCl}_{3}\right): \delta=0.92(\mathrm{~d}, J=6.78 \mathrm{~Hz}, 3 \mathrm{H}), 0.98(\mathrm{~d}, J=$ $6.88 \mathrm{~Hz}, 3 \mathrm{H}), 1.11(\mathrm{~d}, J=6.48 \mathrm{~Hz}, 3 \mathrm{H}), 1.80-1.89(\mathrm{~m}, 1 \mathrm{H}), 3.31-3.40$ (m, 1H), 3.49 (brs, $1 \mathrm{H}), 6.57-$ 
$6.68(\mathrm{~m}, 3 \mathrm{H}), 7.14-7.26(\mathrm{~m}, 2 \mathrm{H}) ;{ }^{13} \mathrm{C} \mathrm{NMR}\left(75 \mathrm{MHz}, \mathrm{CDCl}_{3}\right): \boldsymbol{\delta}=16.5,17.5,19.2,32.1,53.3,112.9$, 116.5, 129.2, 147.8; ESI HRMS exact mass calcd for $\mathrm{C}_{11} \mathrm{H}_{18} \mathrm{~N}$ requires $\mathrm{m} / \mathrm{z} 164.1434$, found $\mathrm{m} / \mathrm{z}$ 164.1431.

The enantiomers were analyzed by HPLC using a chiral OJ-H column ( $n$-heptane/2-Propanol = 99/1, flow rate $=1.0 \mathrm{ml} / \mathrm{min}$, wavelength $=254 \mathrm{~nm}$ ); major enantiomer: $t_{R}=9.84$ min, minor enantiomer: $t_{R}=$ $10.6 \mathrm{~min} ; 91 \%$ ee.

6s: Light yellow oil; Yield: $81 \%$, purification by flash chromatography (2\% EtOAc in Hexanes). ${ }^{1} \mathrm{H}$ $\operatorname{NMR}\left(300 \mathrm{MHz}, \mathrm{CDCl}_{3}\right): \boldsymbol{\delta}=1.42(\mathrm{~d}, J=6.63 \mathrm{~Hz}, 3 \mathrm{H}), 3.73(\mathrm{brs}, 1 \mathrm{H}), 4.16(\mathrm{~m}, 1 \mathrm{H}), 6.26(\mathrm{dd}, J=5.81$ $\mathrm{Hz}, 1 \mathrm{H}), 6.57-6.71(\mathrm{~m}, 4 \mathrm{H}), 7.16-7.39(\mathrm{~m}, 7 \mathrm{H})$;

The enantiomers were analyzed by HPLC using a chiral OJ-H column ( $n$-heptane/2-Propanol = 95/5, flow rate $=1.0 \mathrm{ml} / \mathrm{min}$, wavelength $=254 \mathrm{~nm}$ ); minor enantiomer: $t_{R}=23.48$ min, major enantiomer: $t_{R}=$ $28.93 \mathrm{~min} ; 87 \%$ ee. 
The NMR spectra of $\mathbf{4 a}$

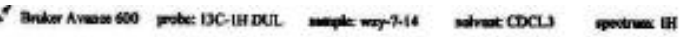

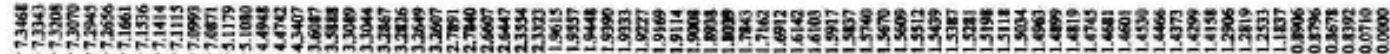<smiles>O=C(NC1CC2C=CC1C2)N1CCCCN1</smiles>

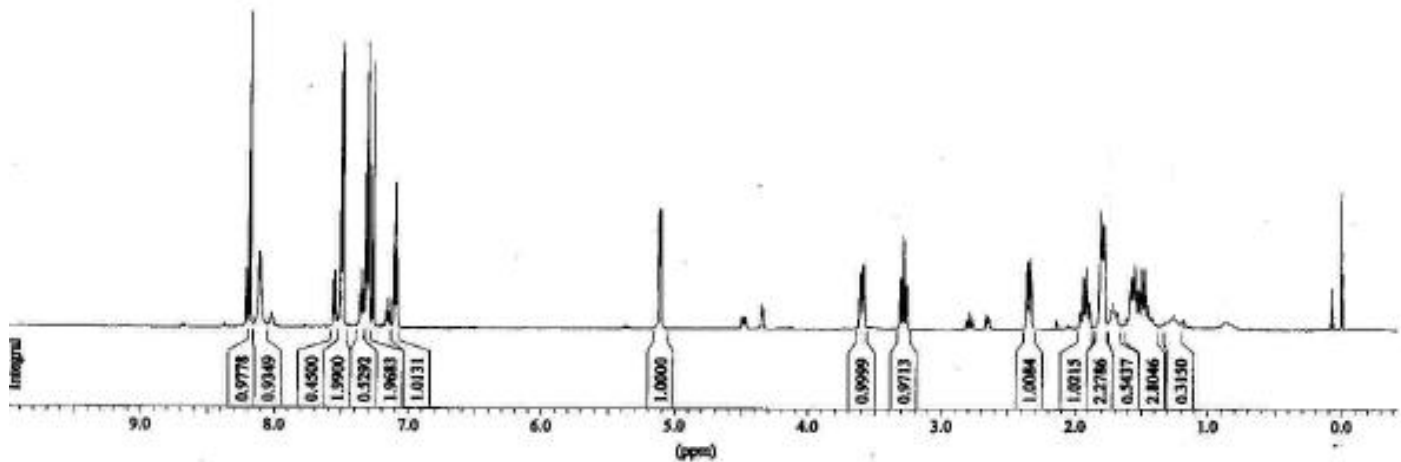

Bnta Amax in pole ISC-IH DUR.

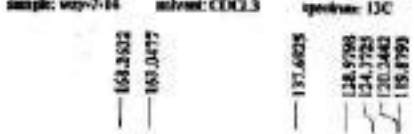

醀

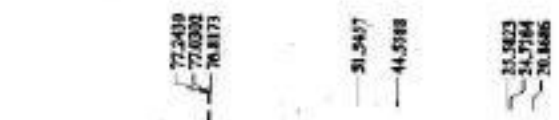
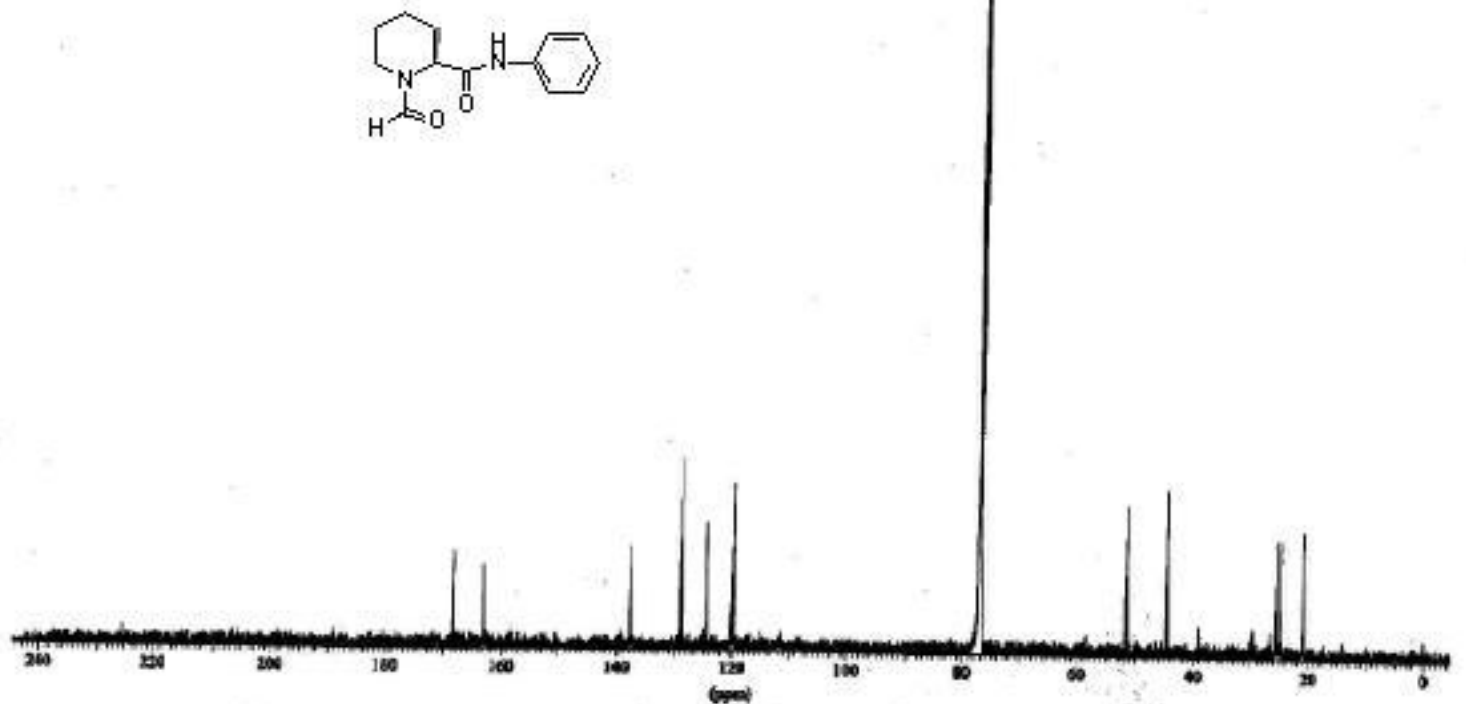
The NMR spectra of $\mathbf{4 b}$

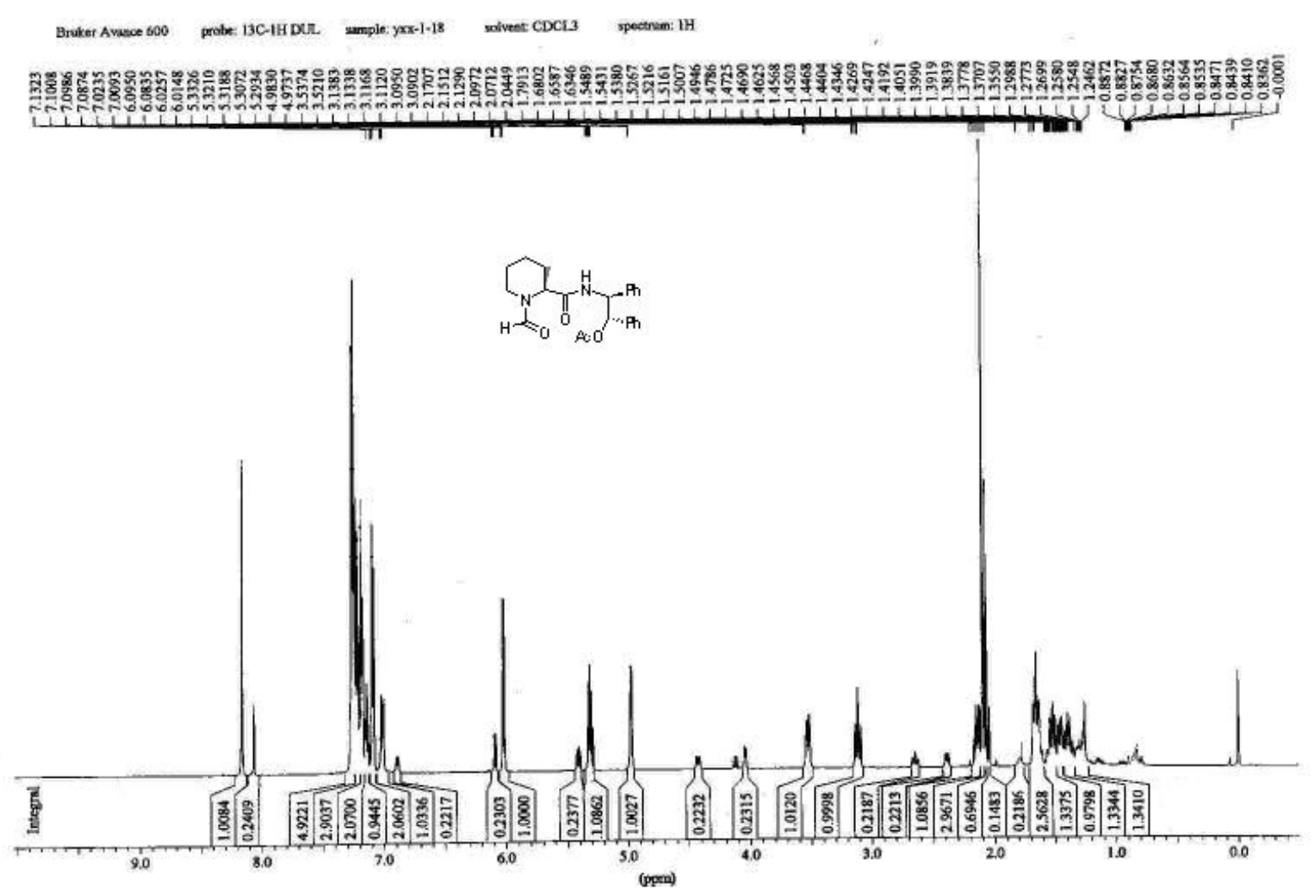

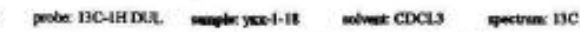

ว

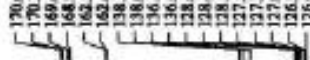

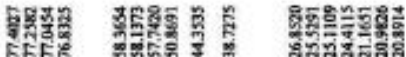

ivi |

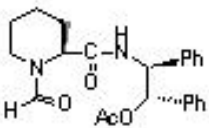

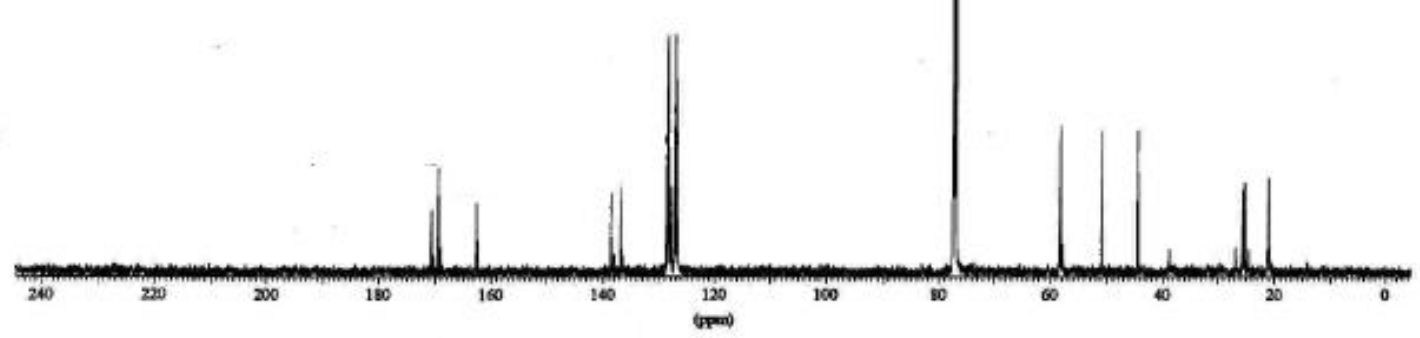


The NMR spectra of $\mathbf{4 c}$

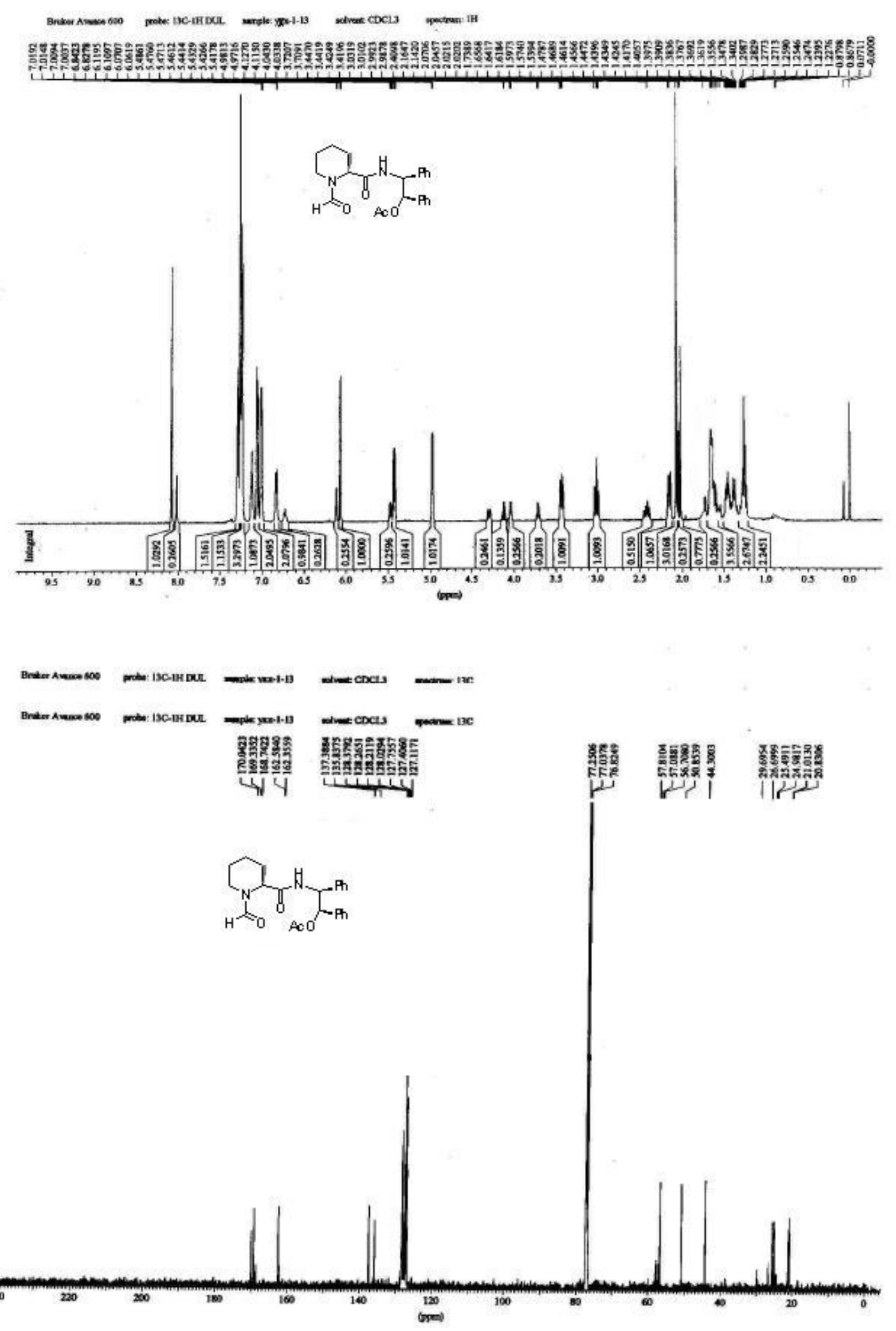


The NMR spectra of $\mathbf{4 d}$
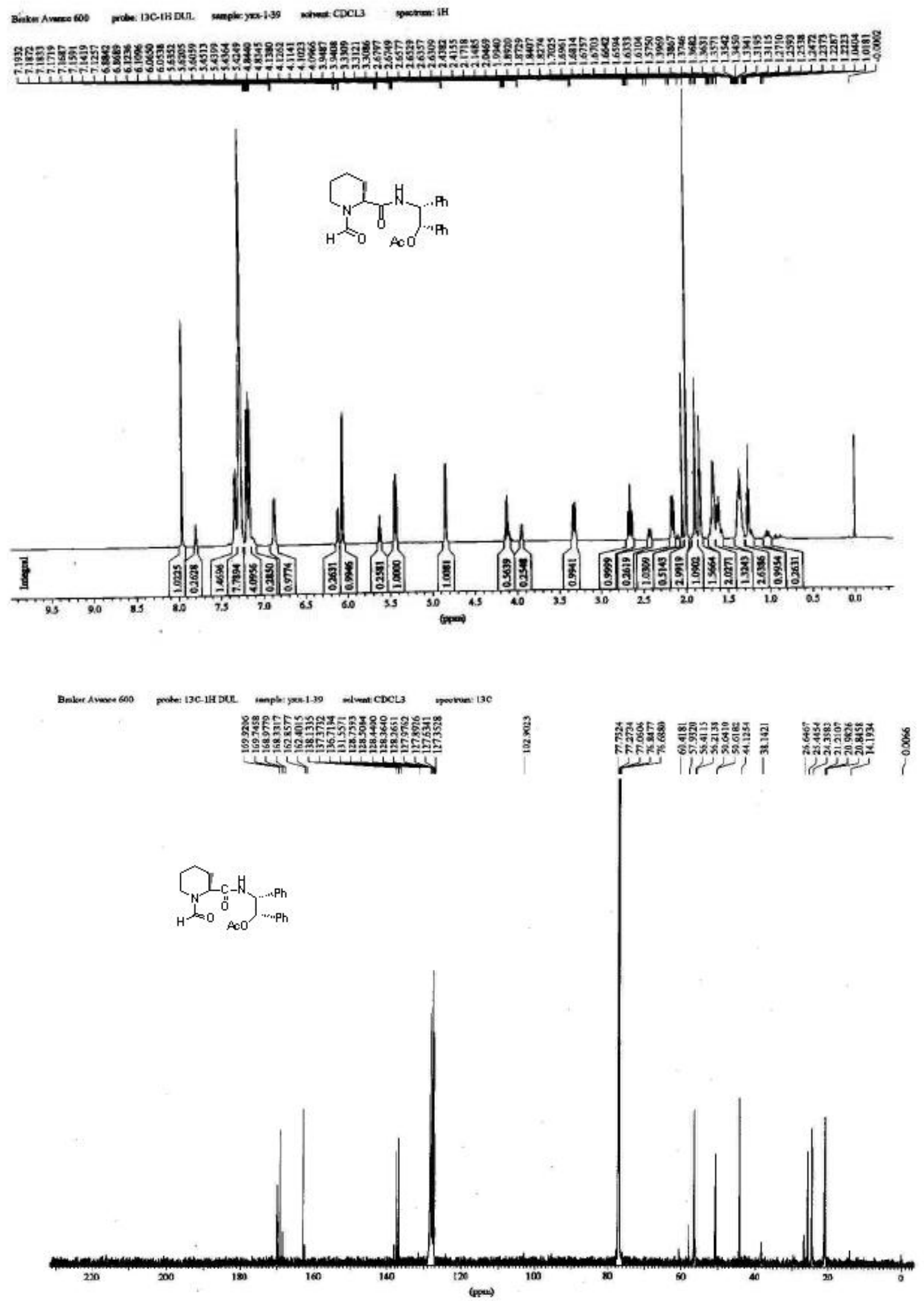
The NMR spectra of $\mathbf{5 d}$

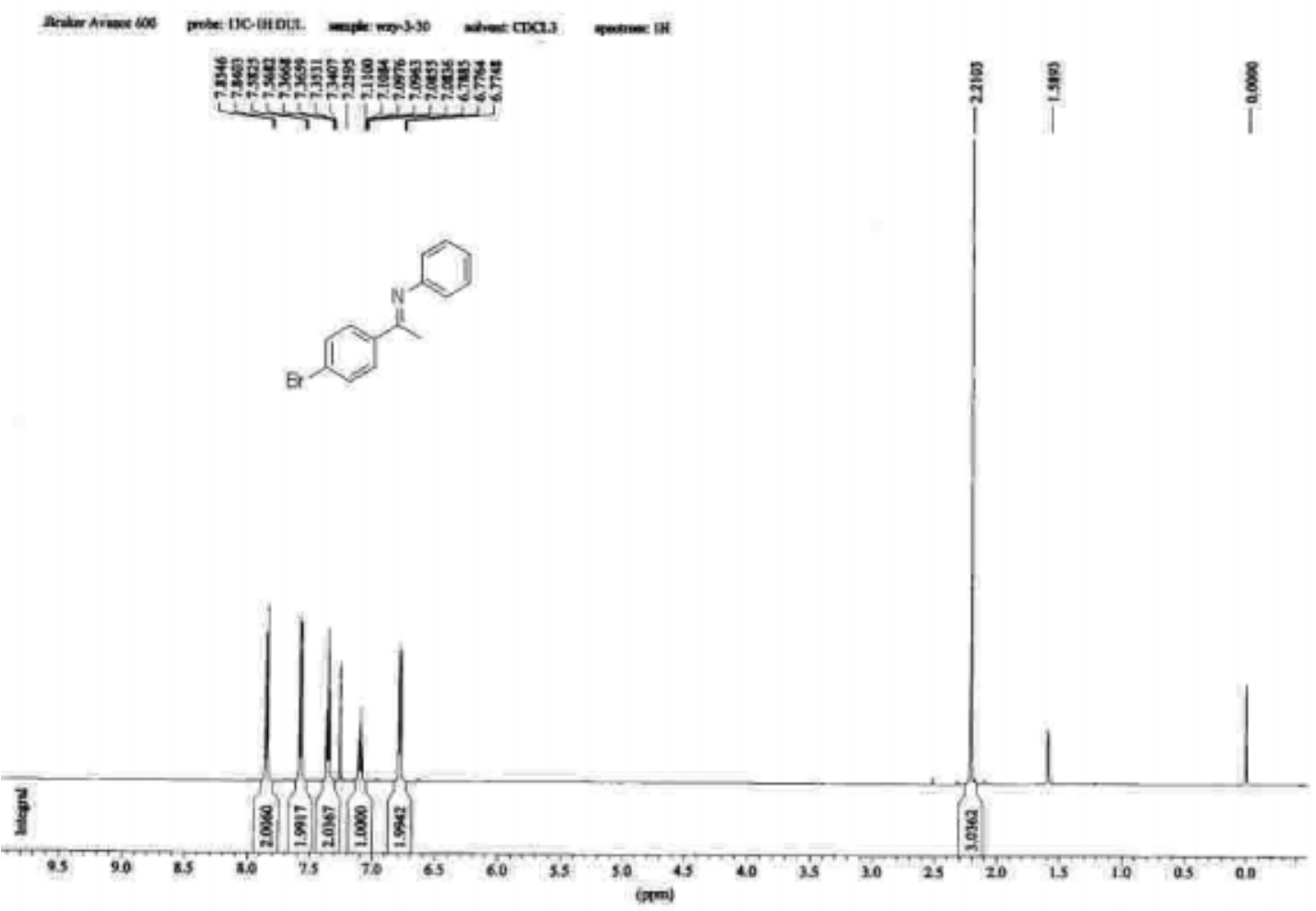

$n x-3-15$

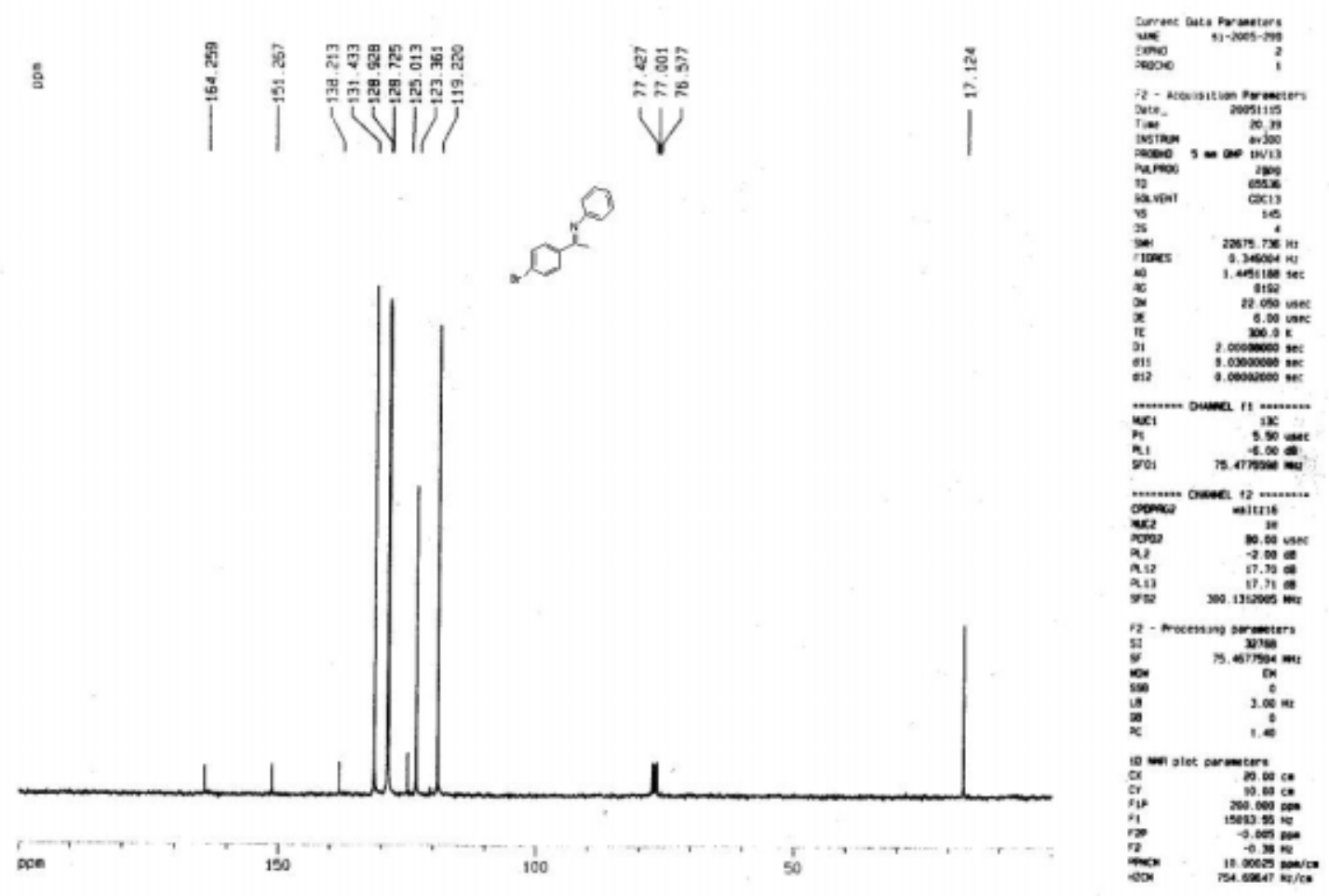


The NMR spectra of $\mathbf{5 e}$

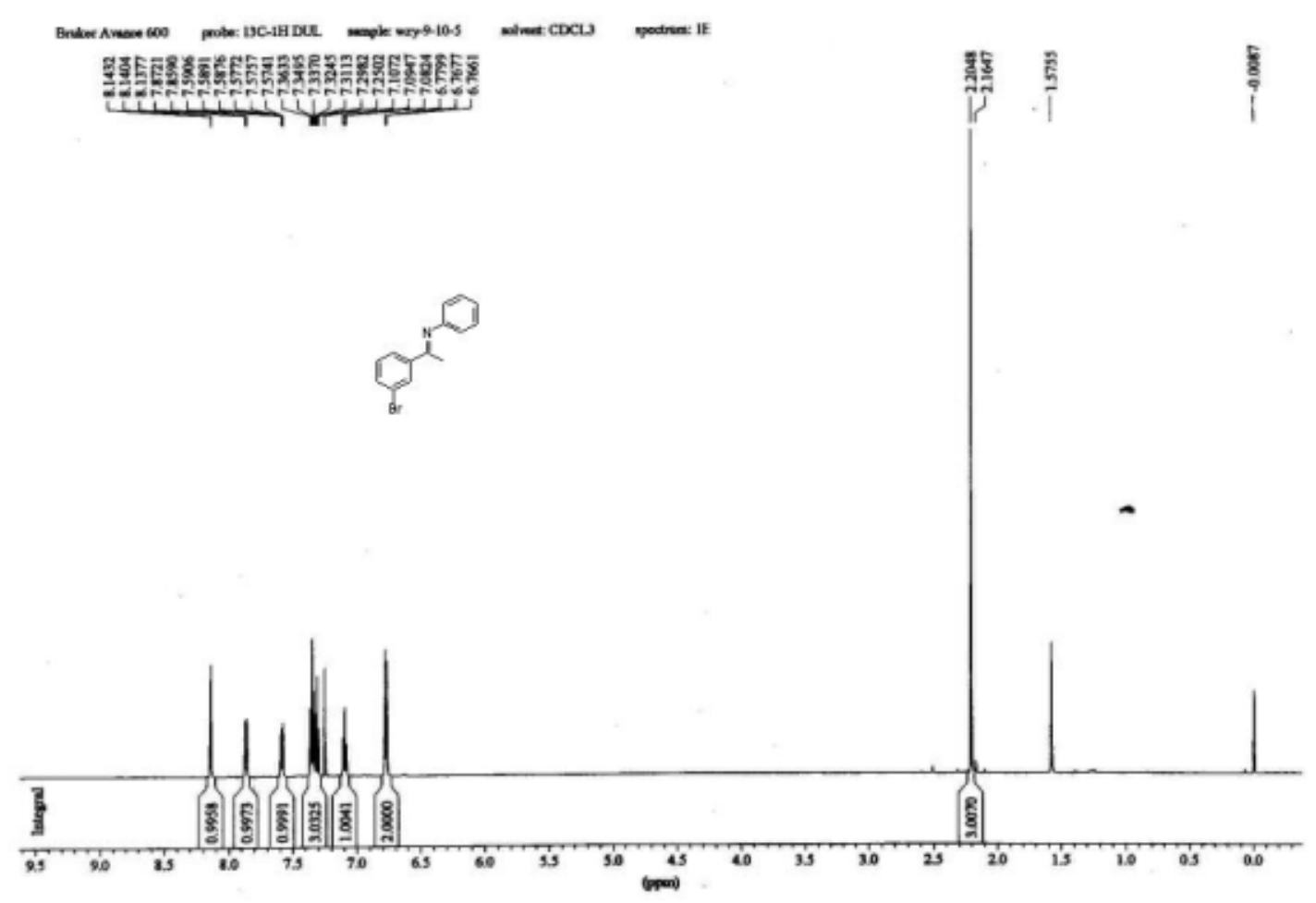

w2 $\gamma-9-10-5$

帘

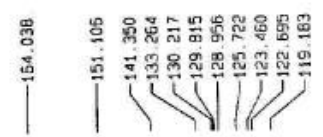

พู่รั้ำ

a

if

V

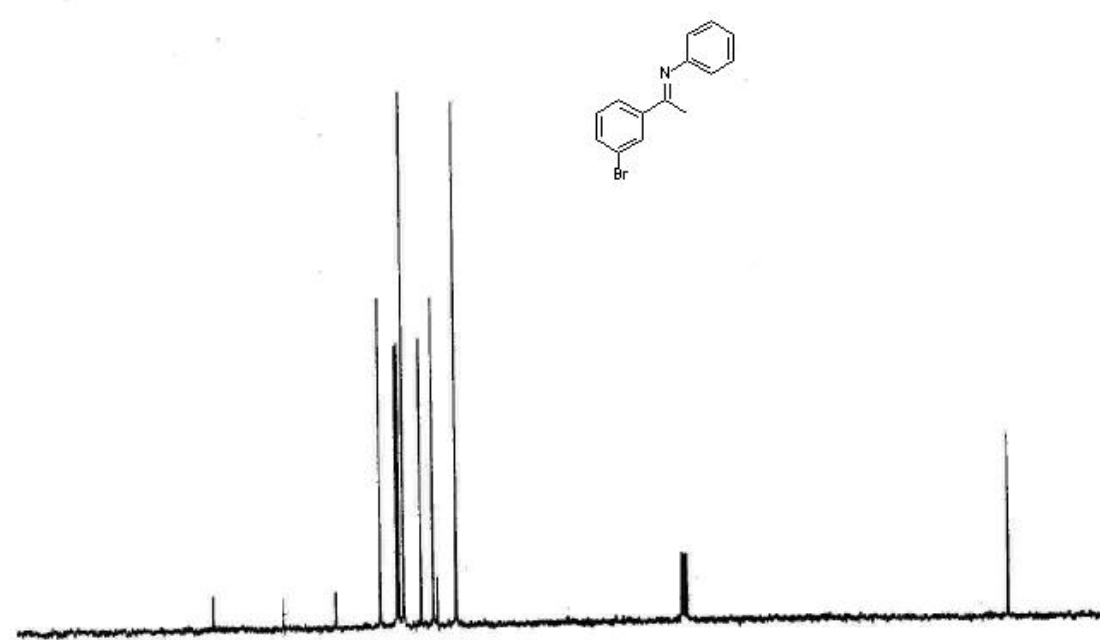

pom

${ }_{100}^{7}$

50

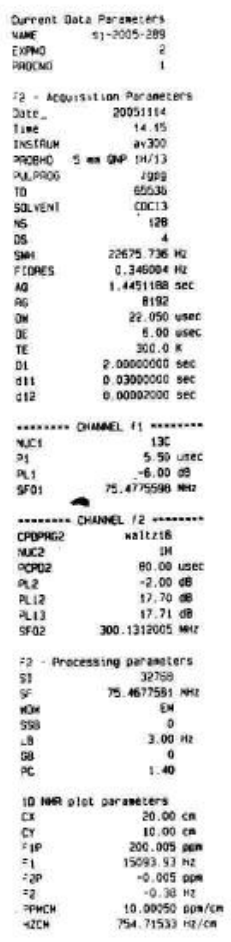


The NMR spectra of $\mathbf{5 h}$

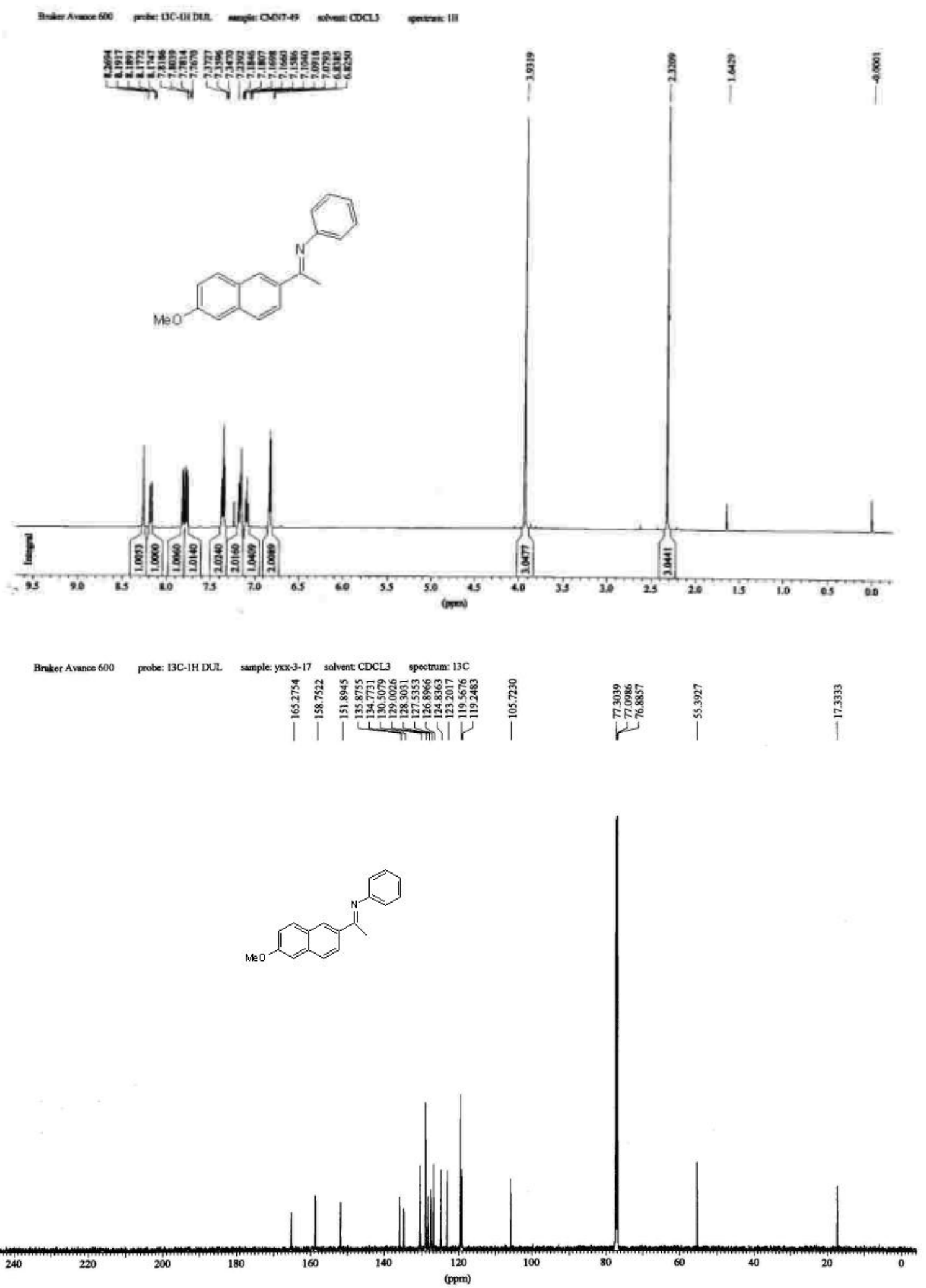


The NMR spectra of $\mathbf{5 l}$

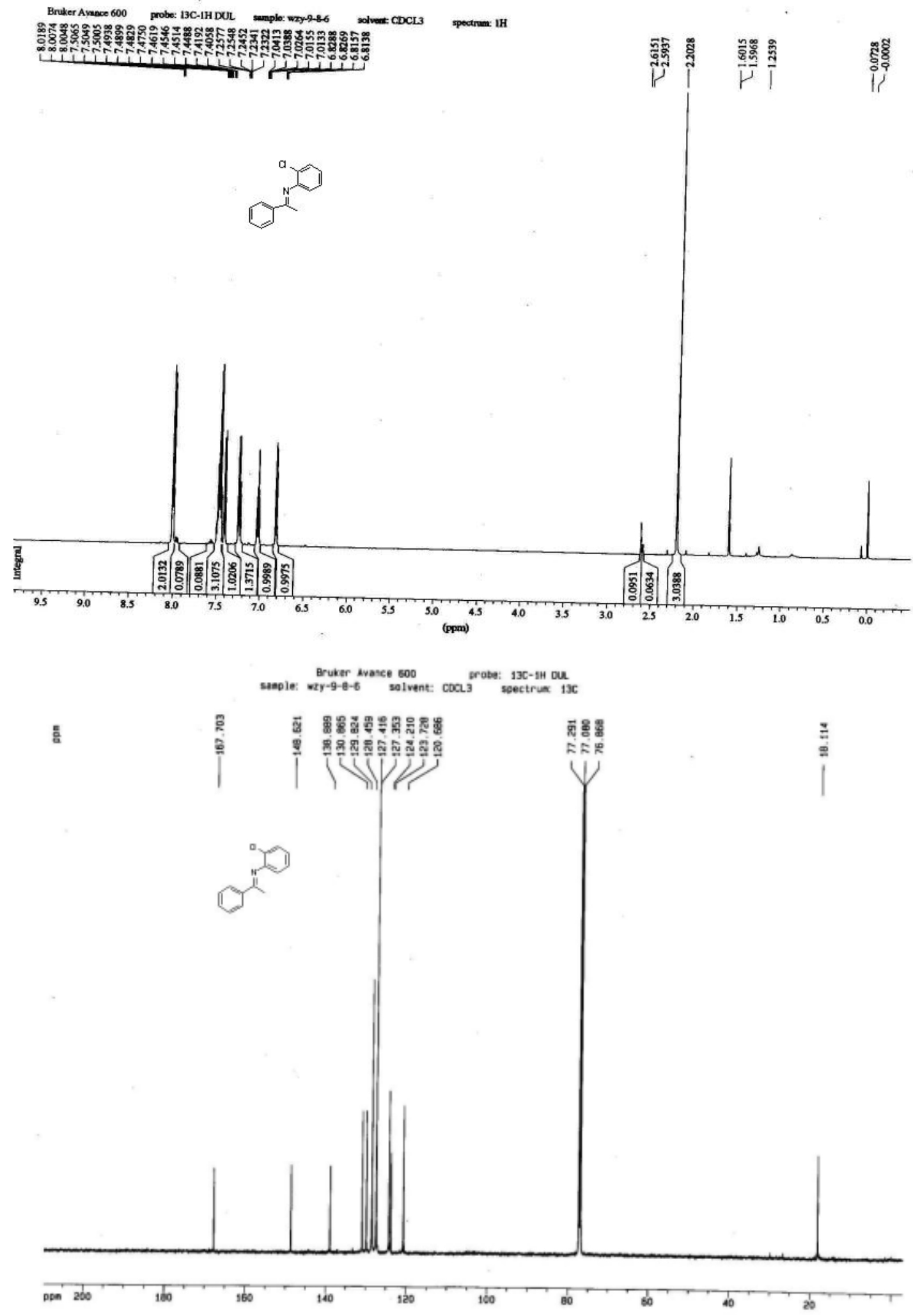


The NMR spectra of $\mathbf{5 m}$

Bruker Avanoe 600 probe: $13 \mathrm{C}-1 \mathrm{HDU}$. sample wzy-9-10-2 solvent: CDCL3 spectrum: If

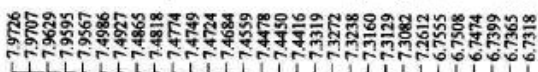<smiles>CC(=Nc1ccc(Cl)cc1)c1ccccc1</smiles>

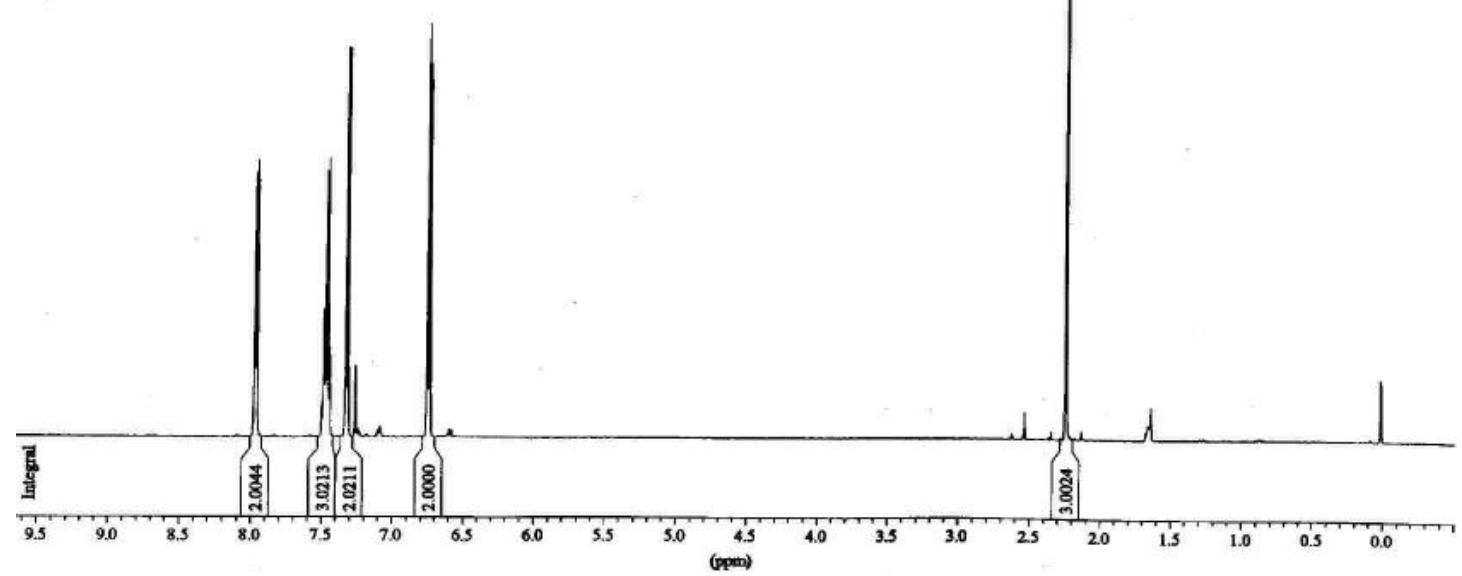

w2y-9-10-2

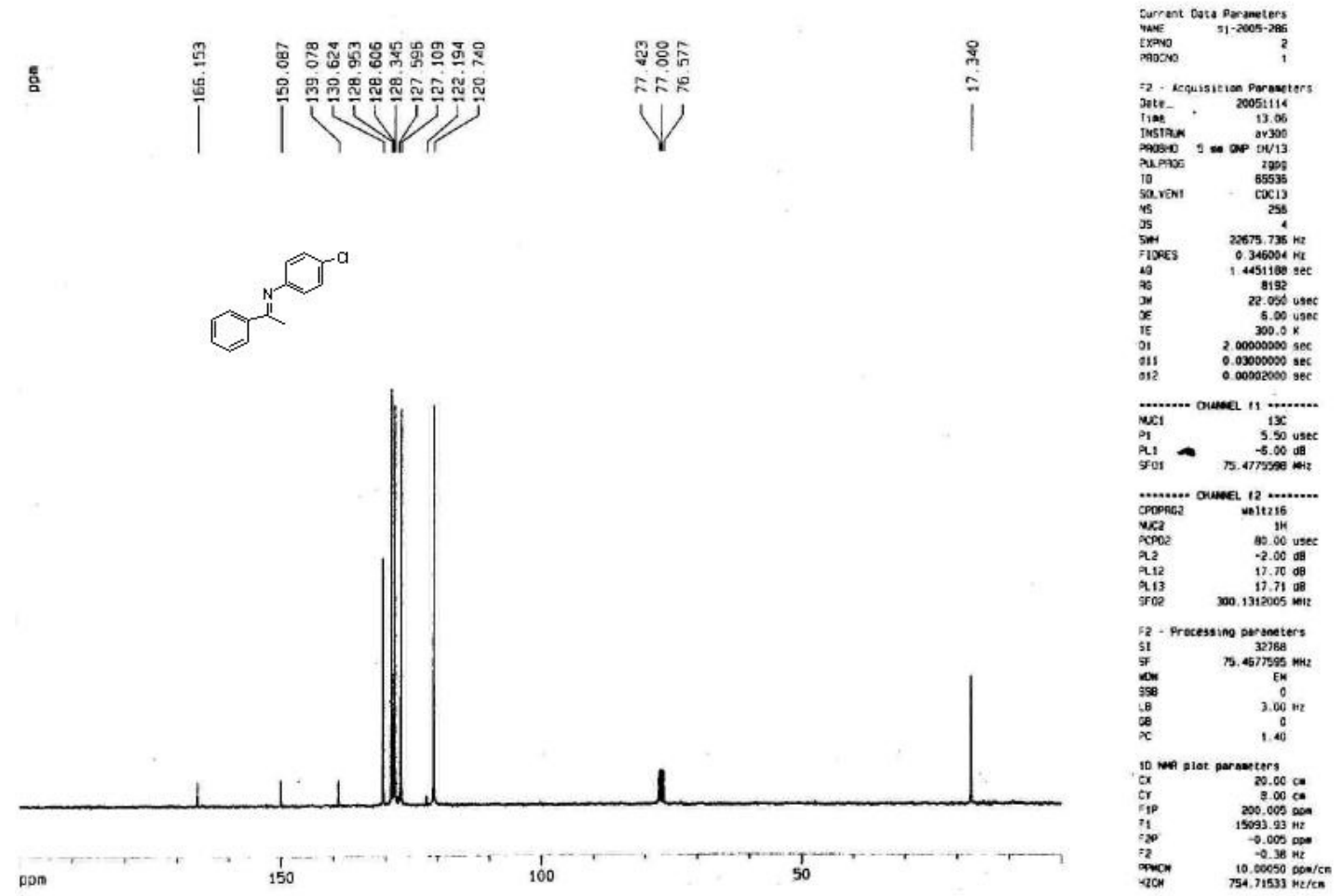



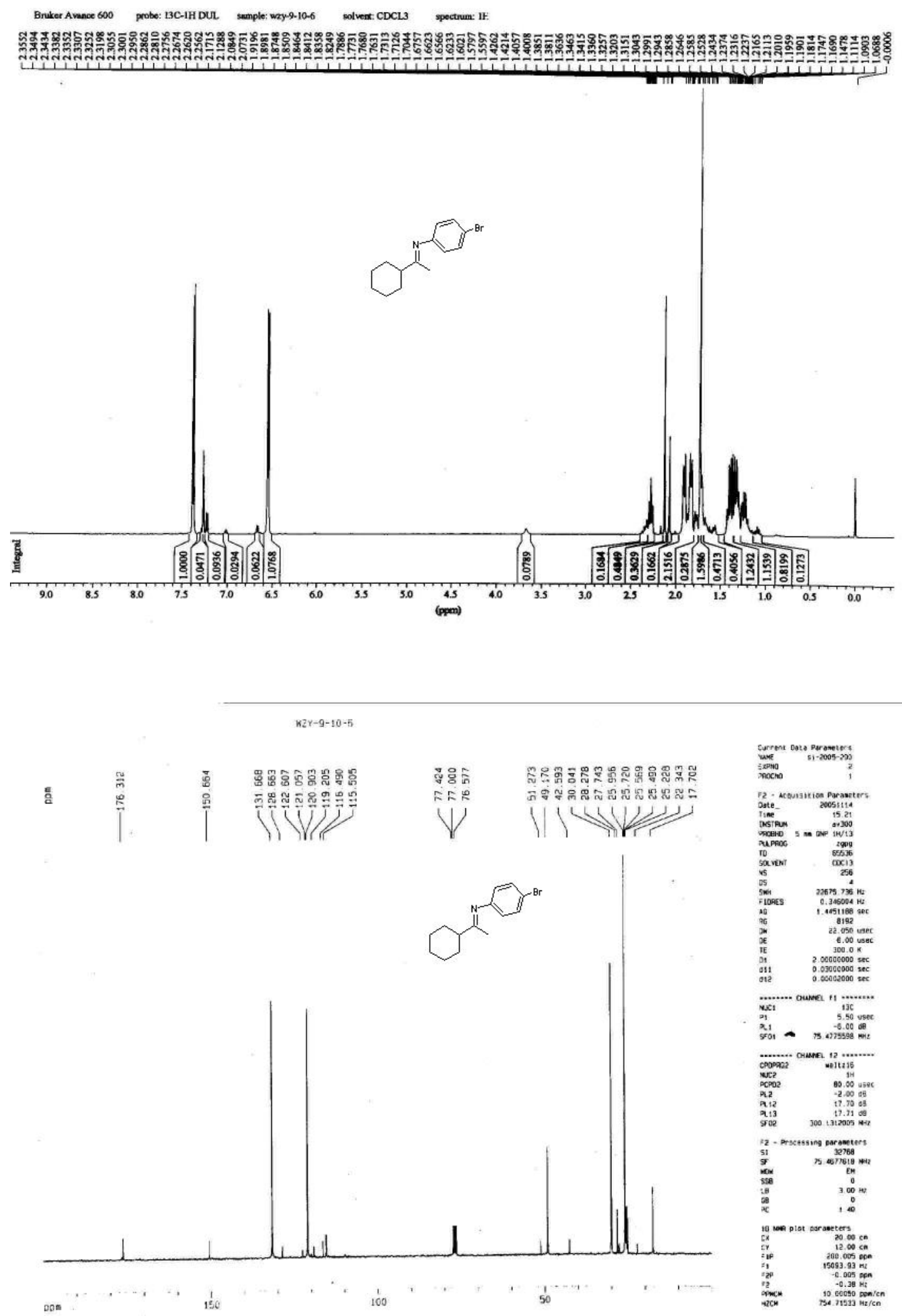
The NMR spectra of $\mathbf{5 q}$

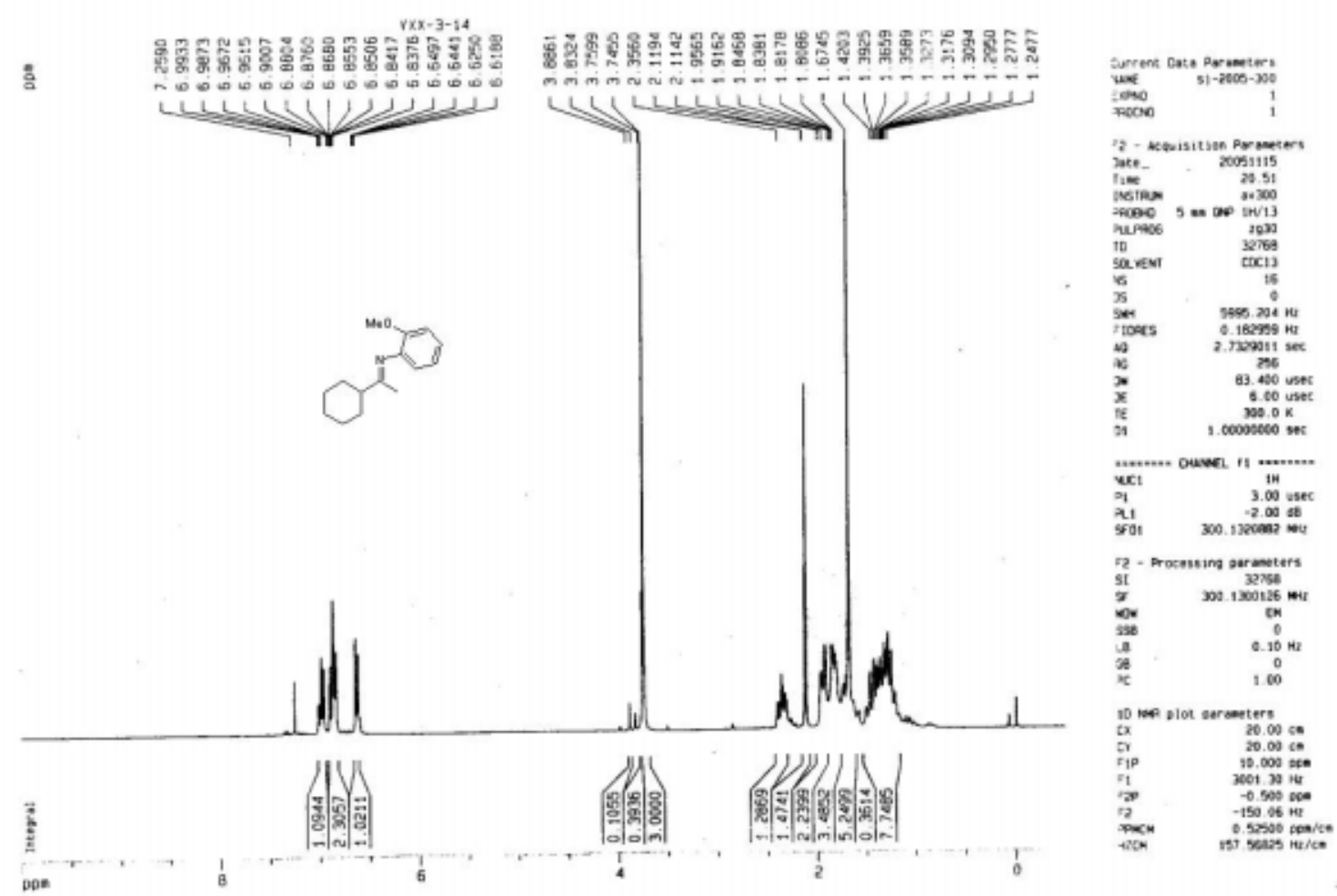

$x \times x-3-14$

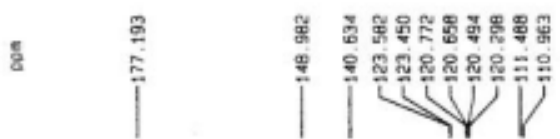

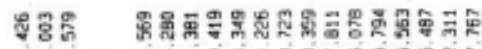

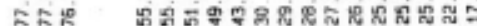

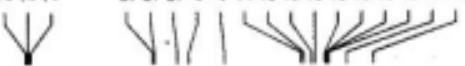
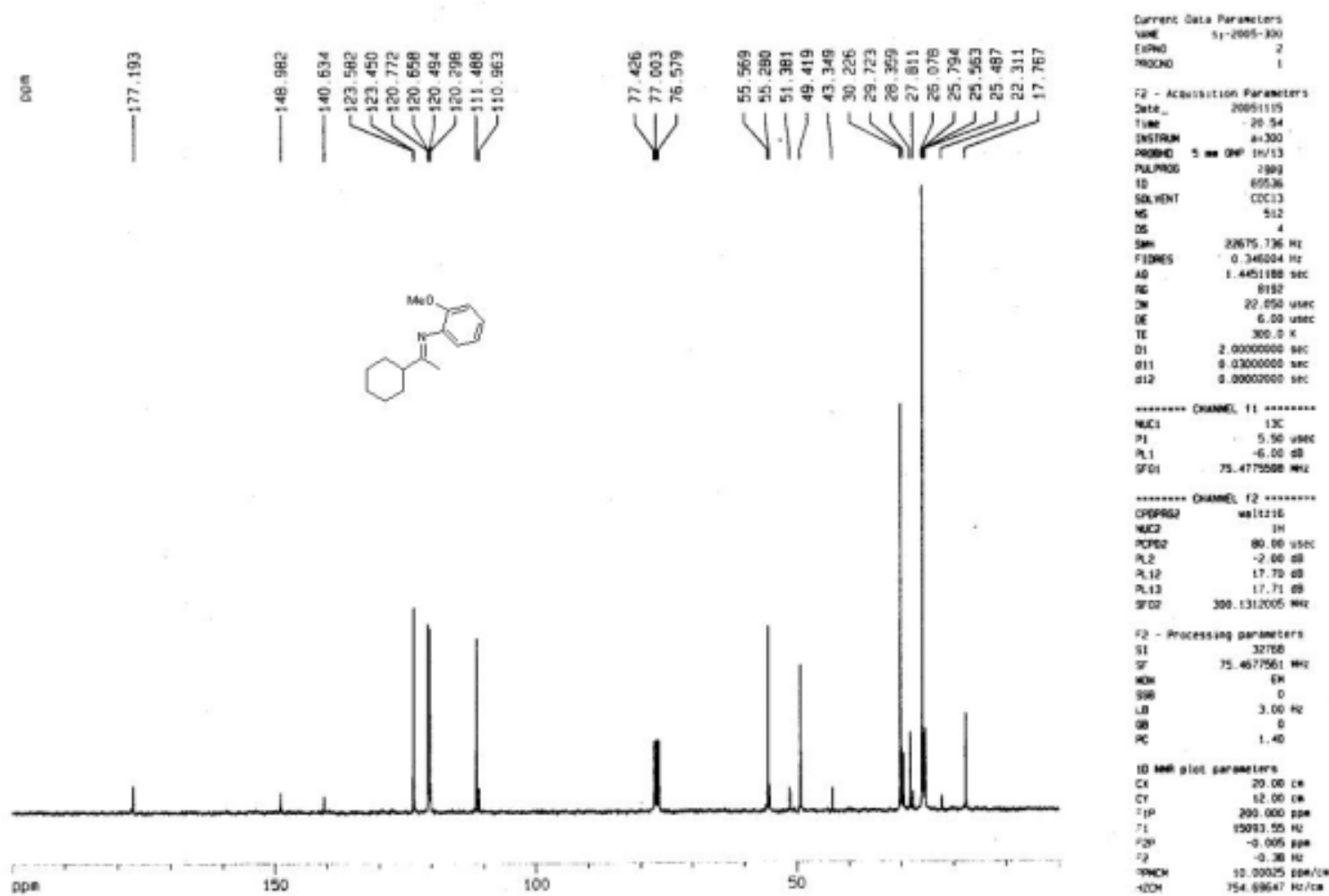
The NMR spectra of $\mathbf{5 r}$
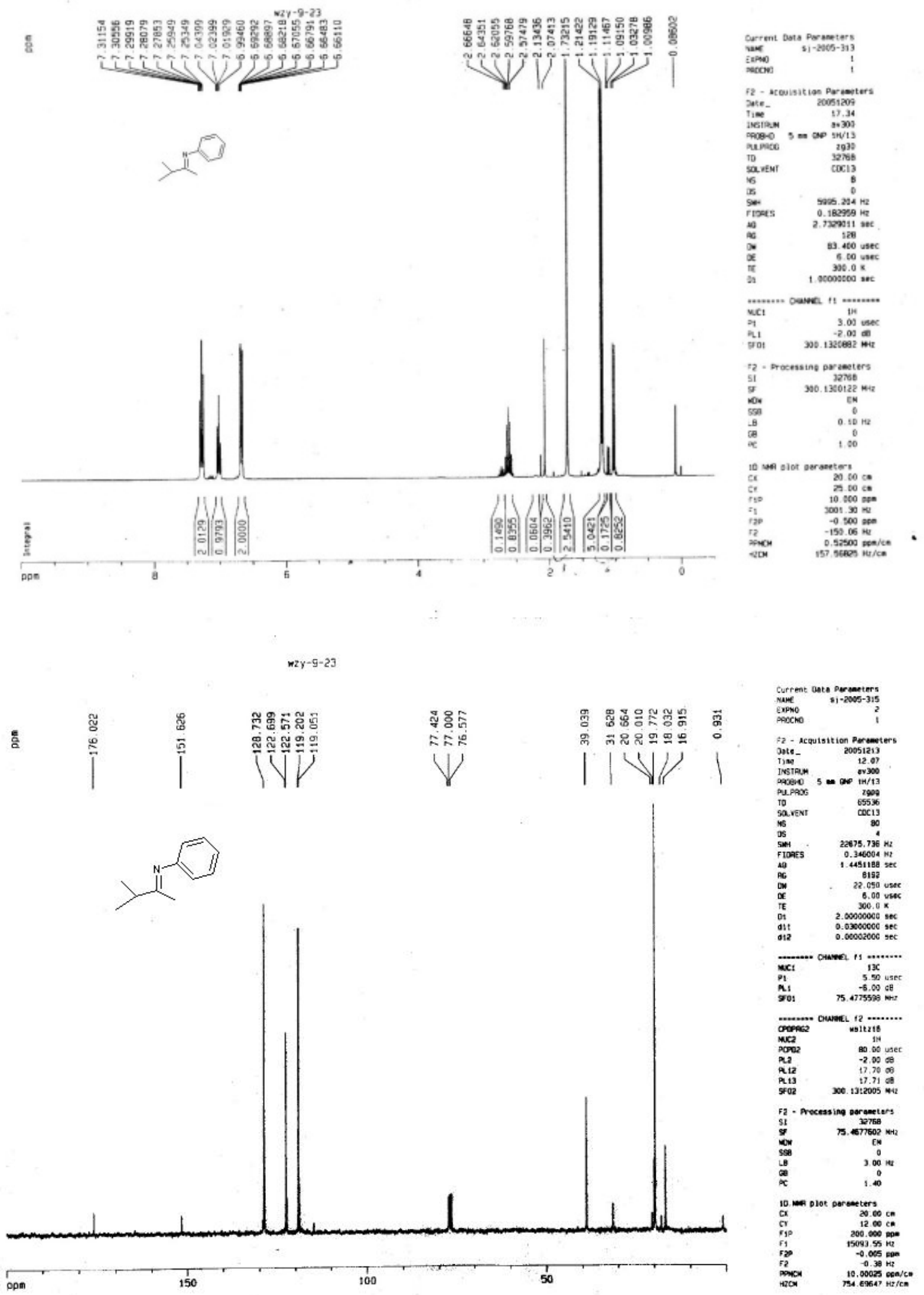
The NMR spectra of $\mathbf{6 a}$

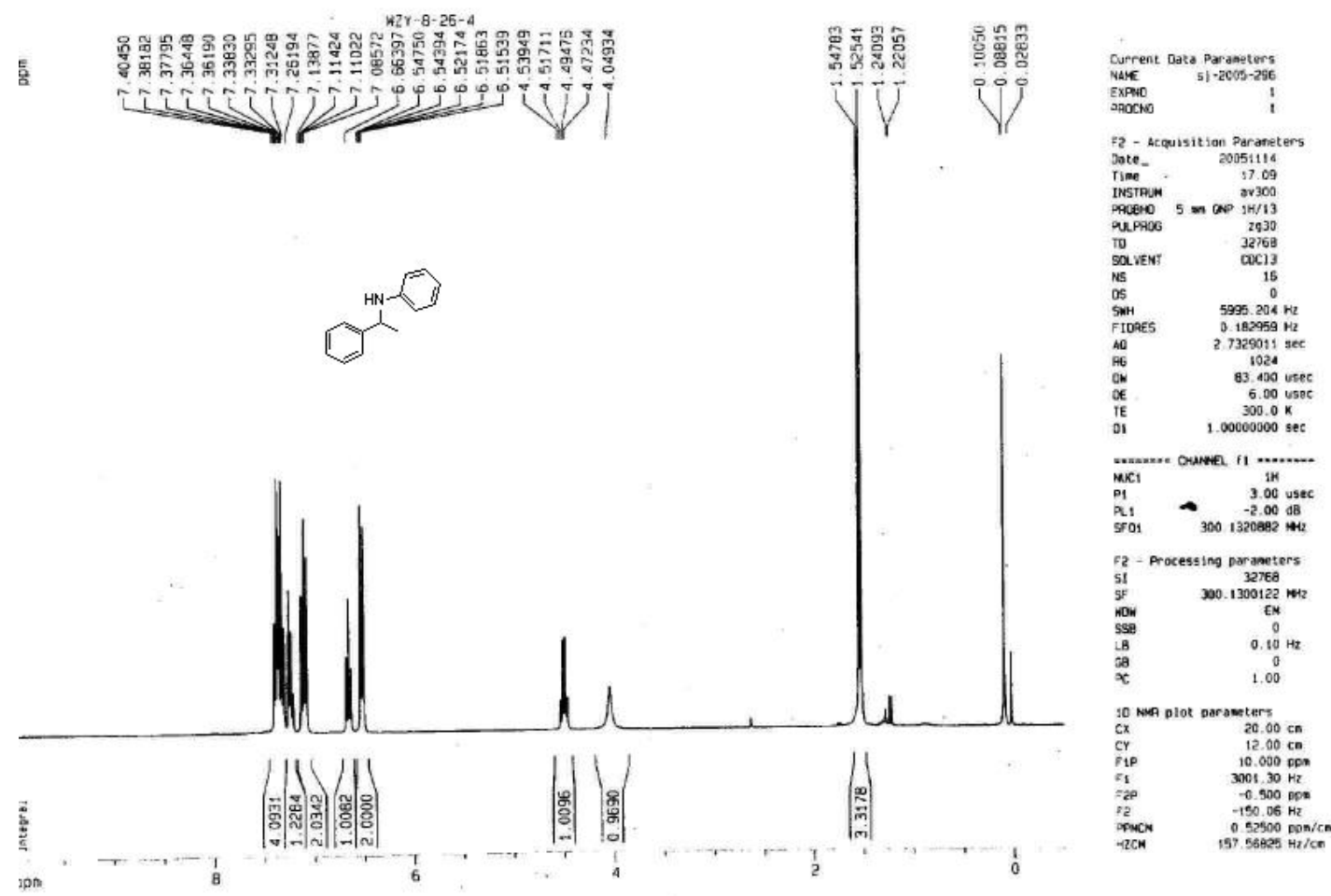

The NMR spectra of $\mathbf{6 b}$

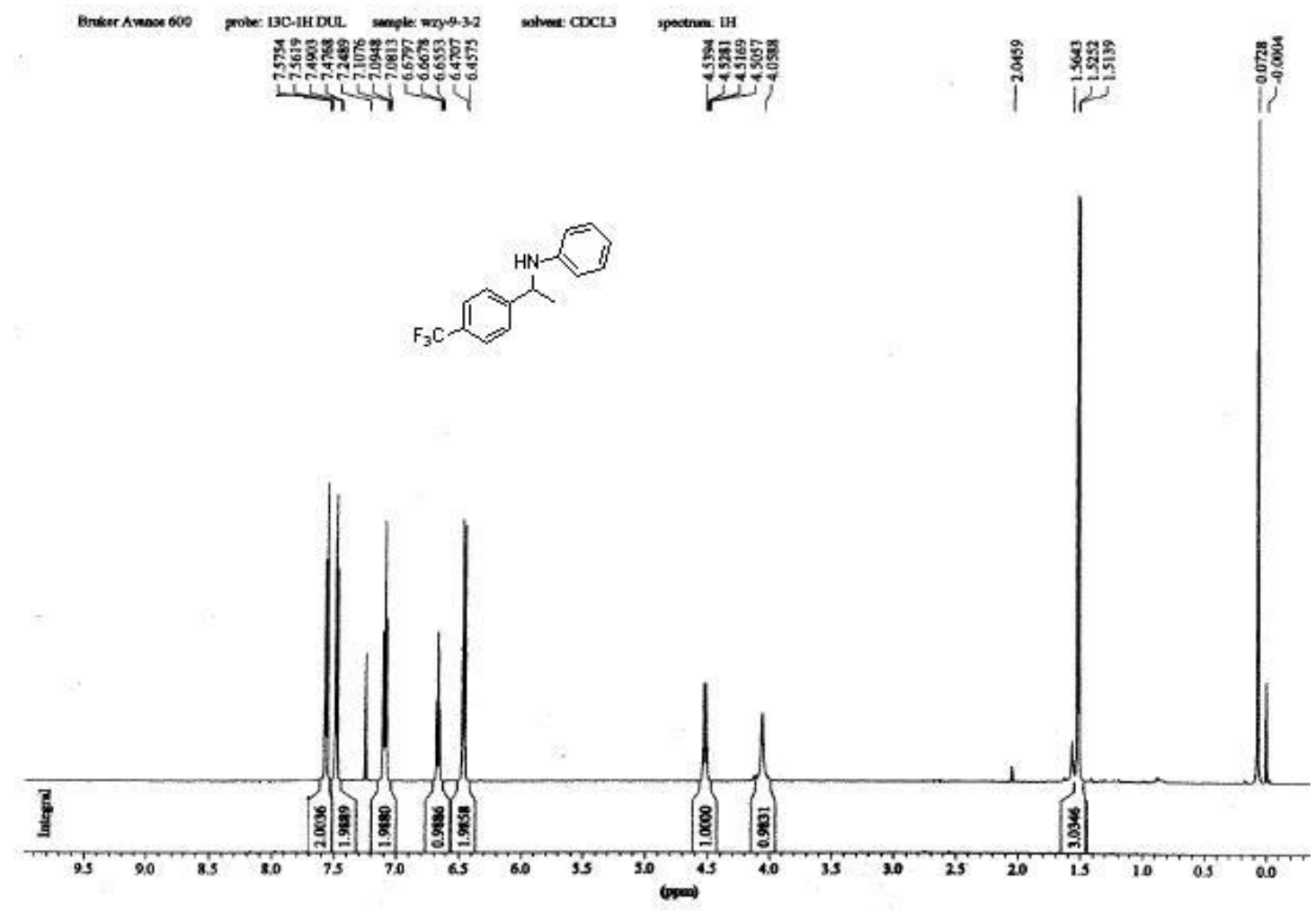


I

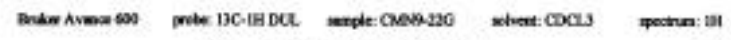

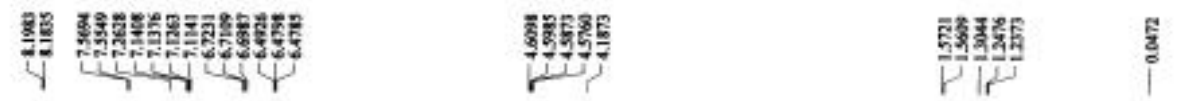<smiles>CC(Nc1ccccc1)c1ccc([N+](=O)[O-])cc1</smiles>

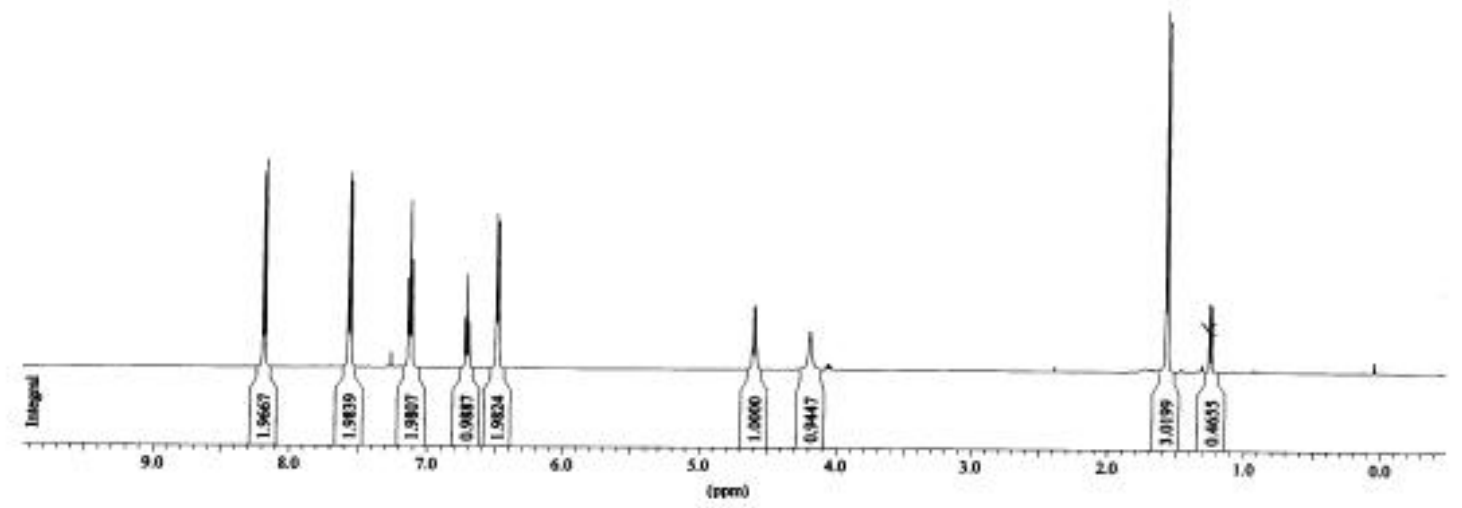


The NMR spectra of $\mathbf{6 d}$

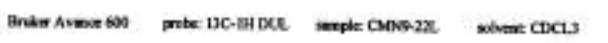

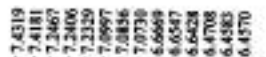

$4+4$

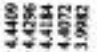

等

裇<smiles>CC(Nc1ccccc1)c1ccc(Br)cc1</smiles>
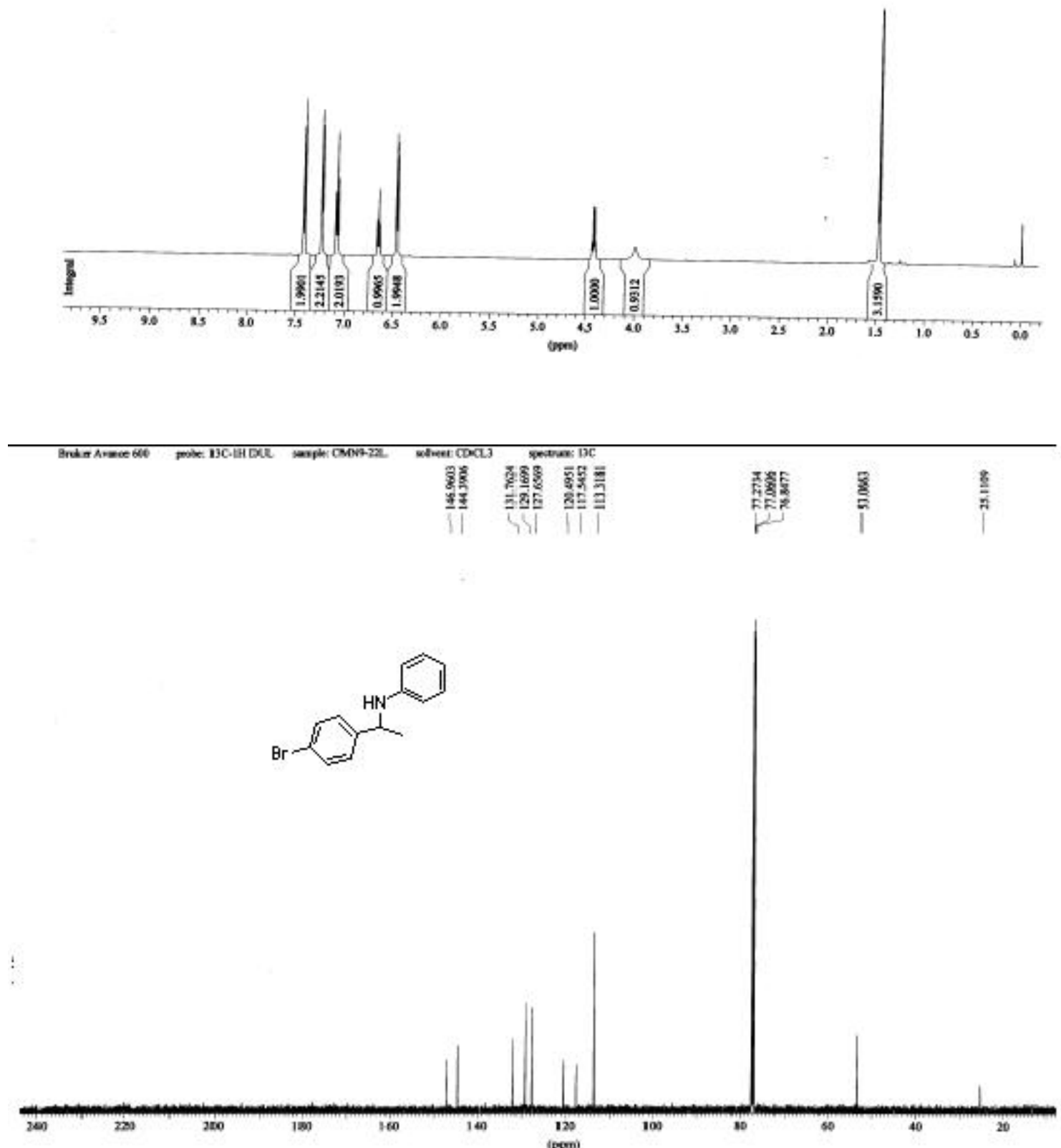
The NMR spectra of $\mathbf{6 e}$
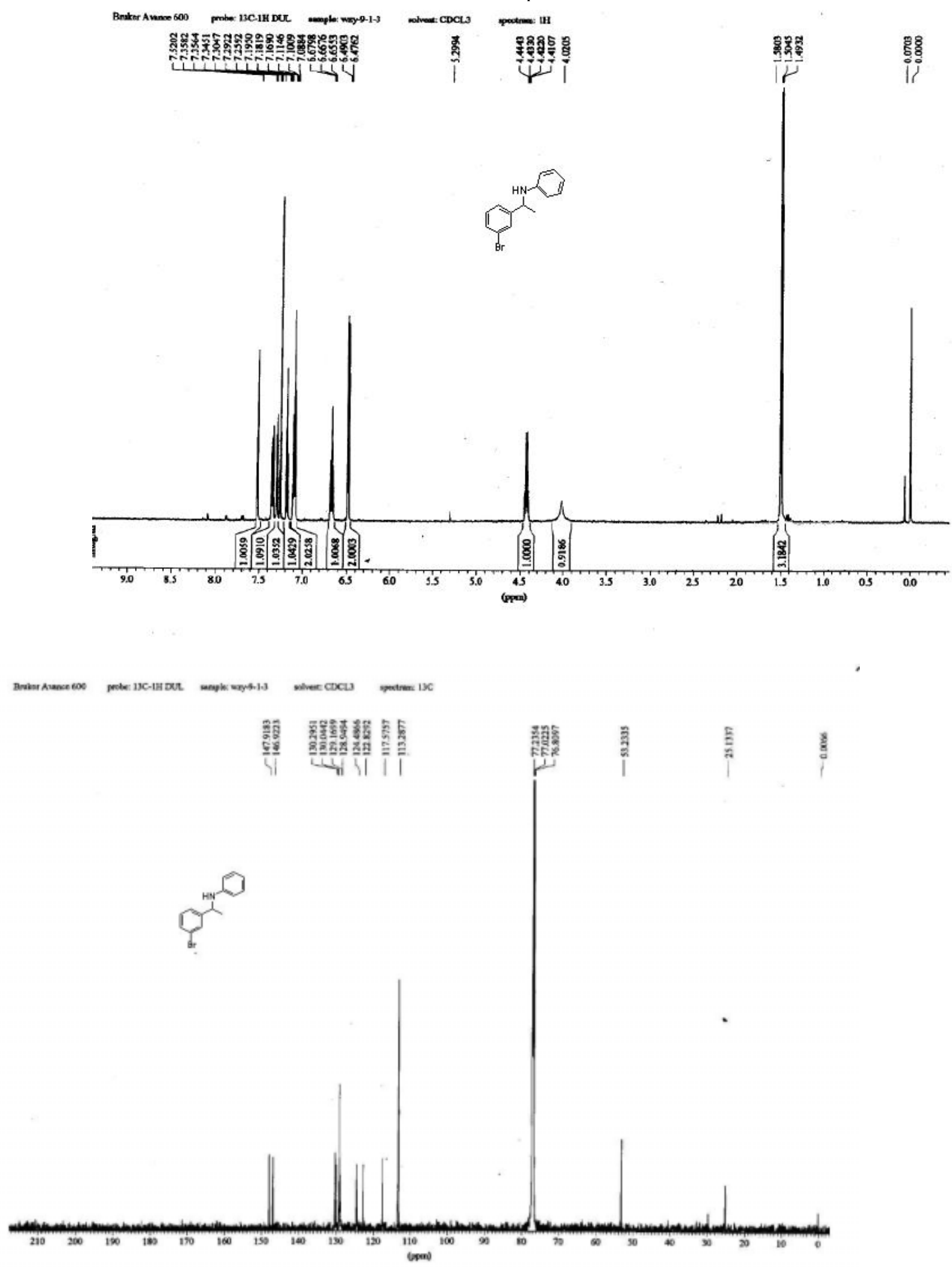
The NMR spectra of $\mathbf{6 f}$

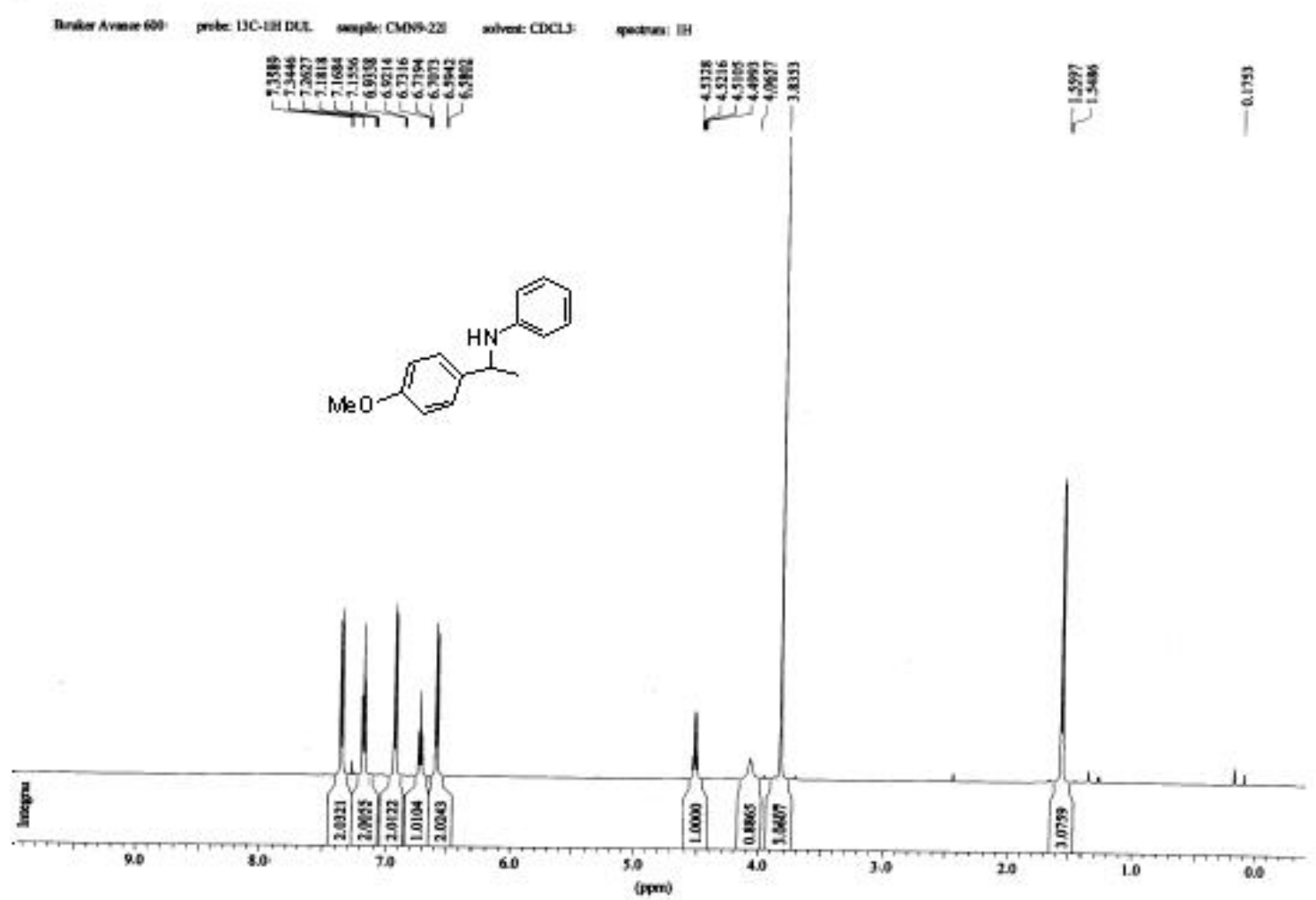

The NMR spectra of $\mathbf{6} \mathbf{g}$
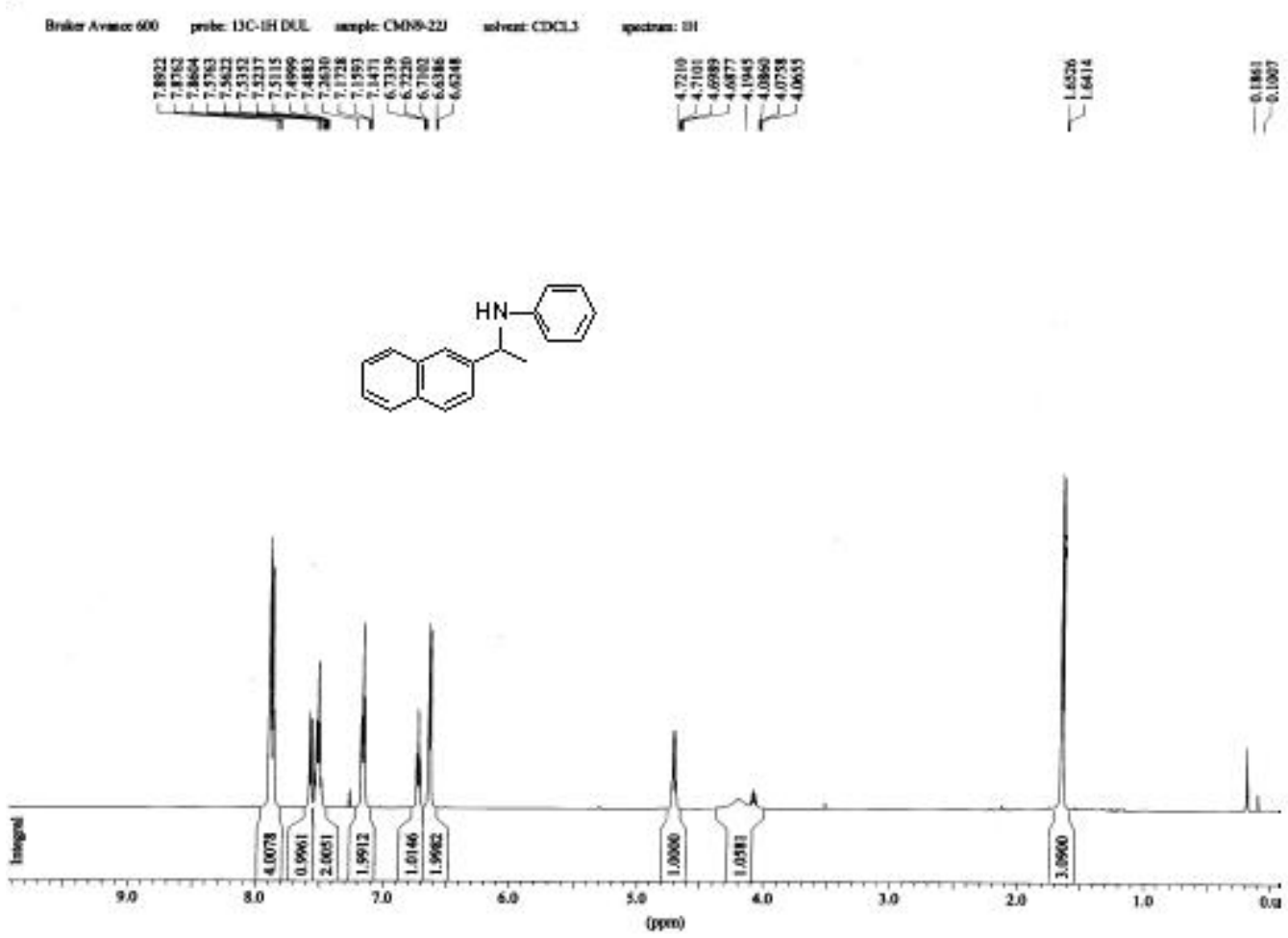
The NMR spectra of $\mathbf{6 h}$
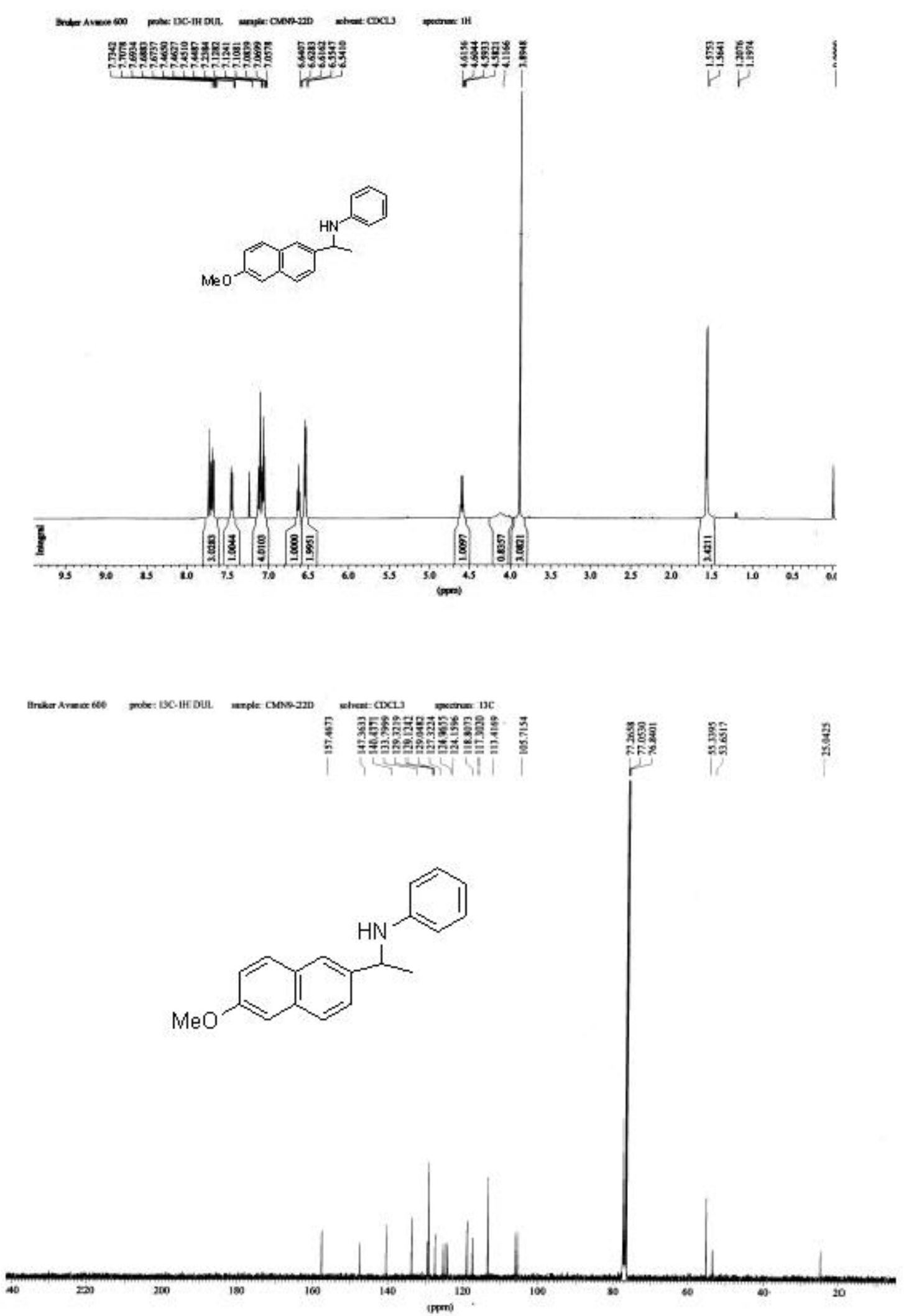

The NMR spectra of $\mathbf{6 i}$ 


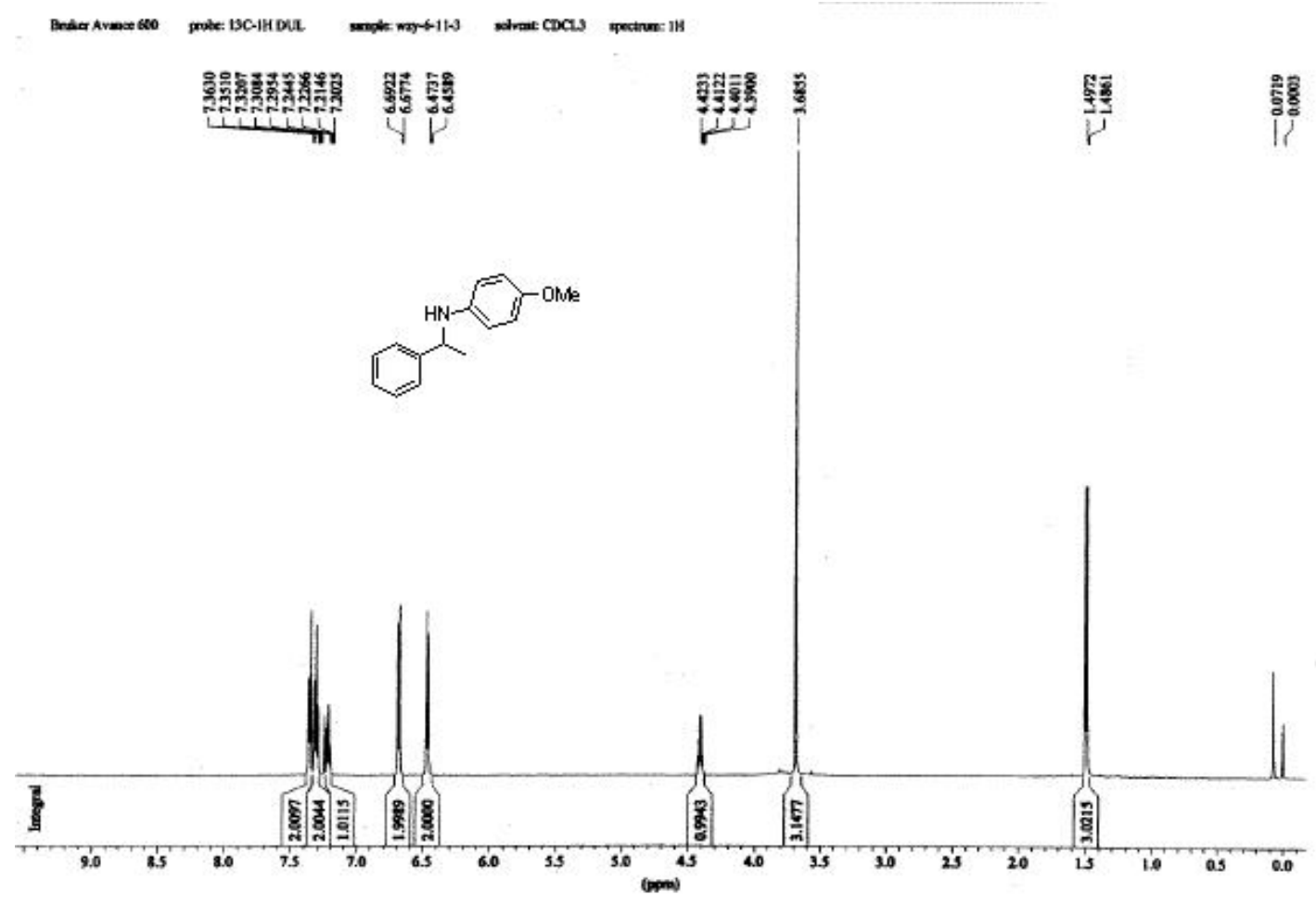


The NMR spectra of $\mathbf{6 j}$
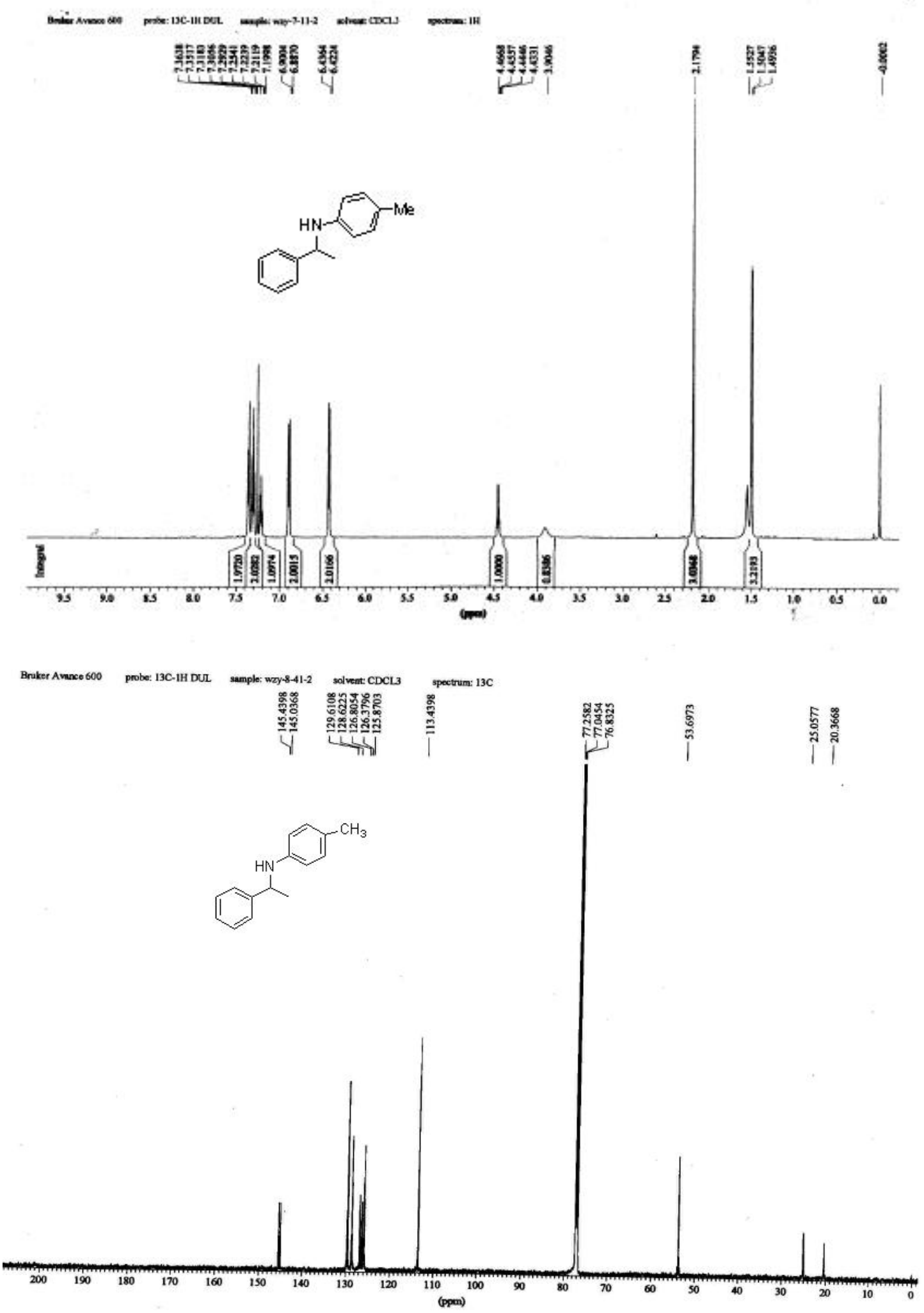
The NMR spectra of $\mathbf{6 k}$

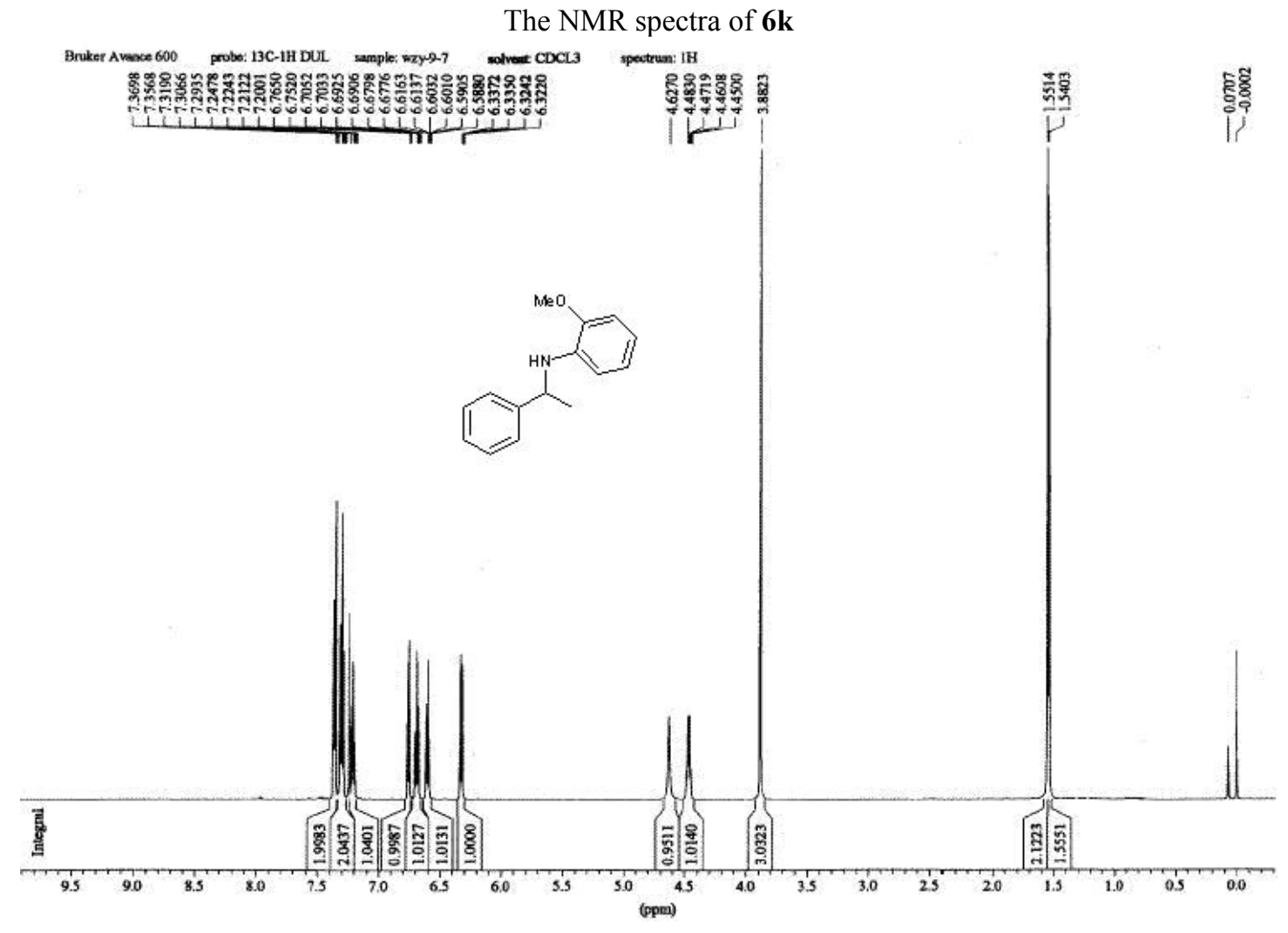


The NMR spectra of $\mathbf{6} \mathbf{I}$

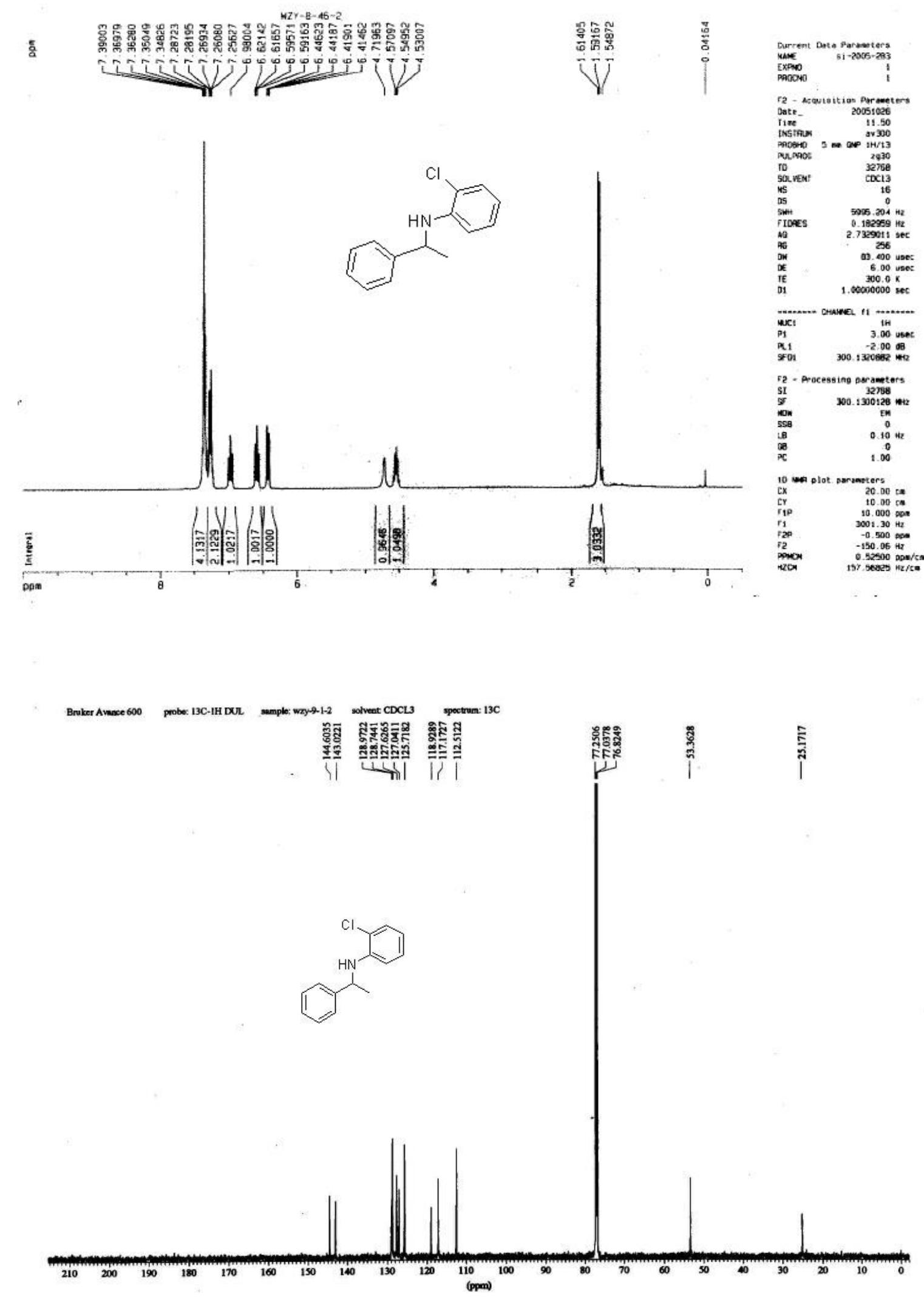


The NMR spectra of $\mathbf{6 m}$

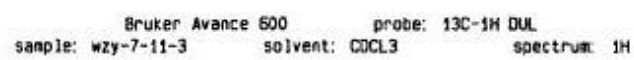
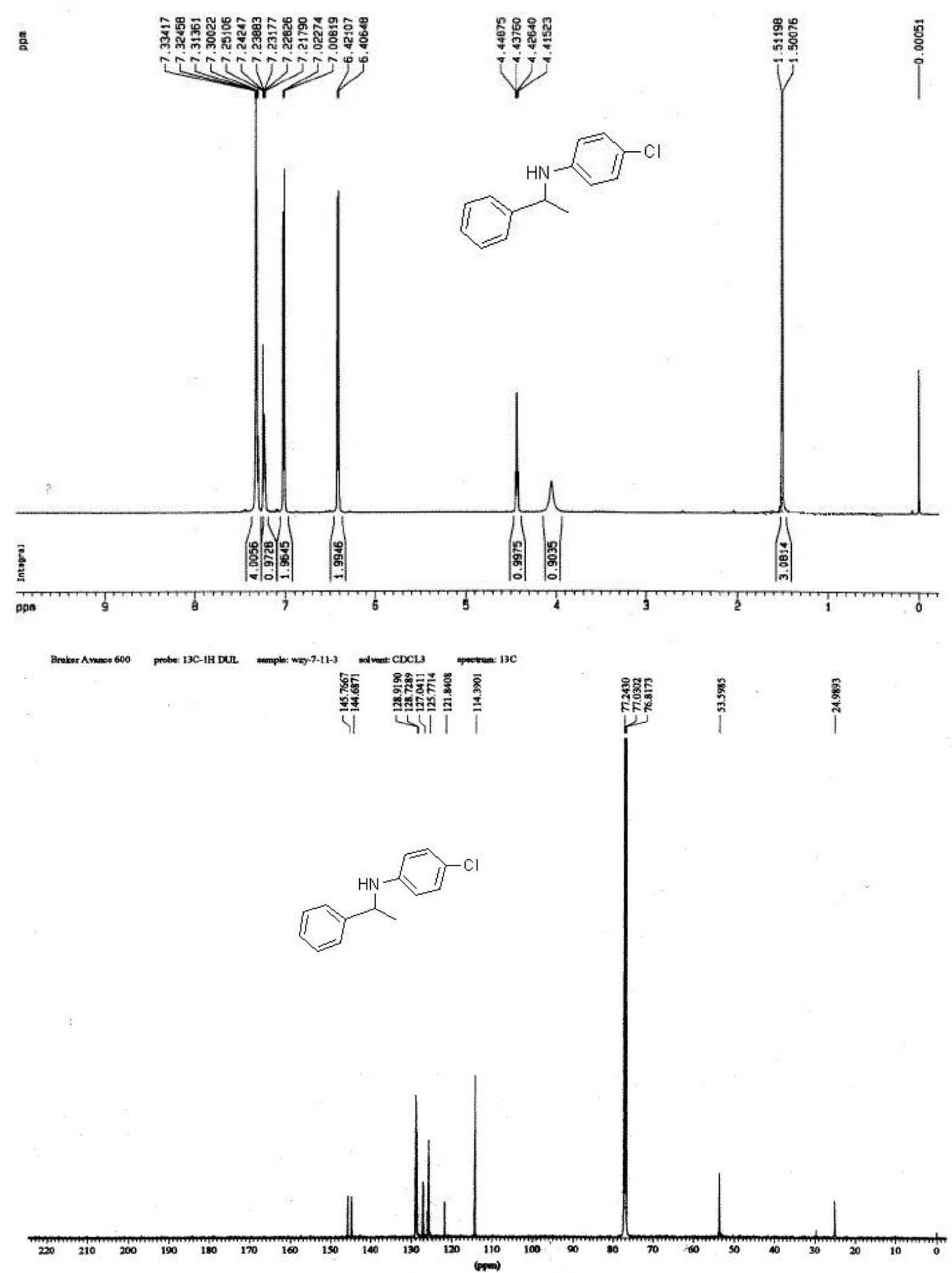
The NMR spectra of $\mathbf{6 n}$
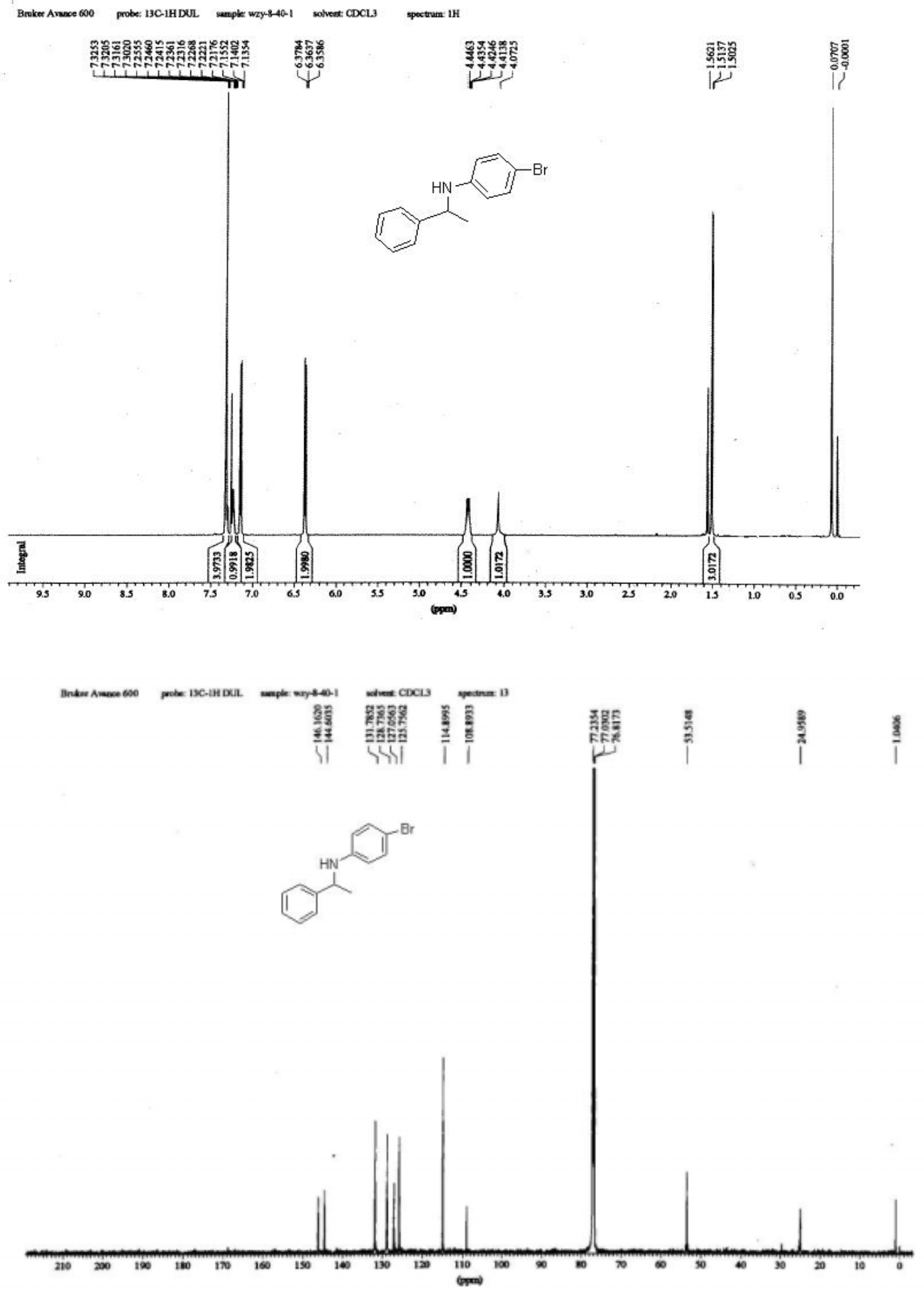
The NMR spectra of $\mathbf{6 0}$

Aruter Anawe 600 probe $13 C-18$ DUL.

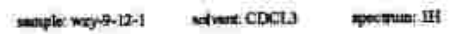

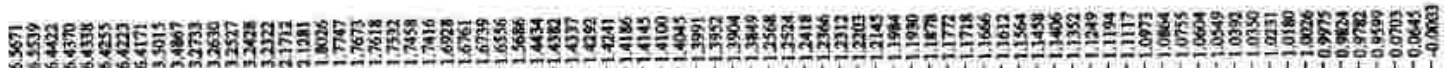

i
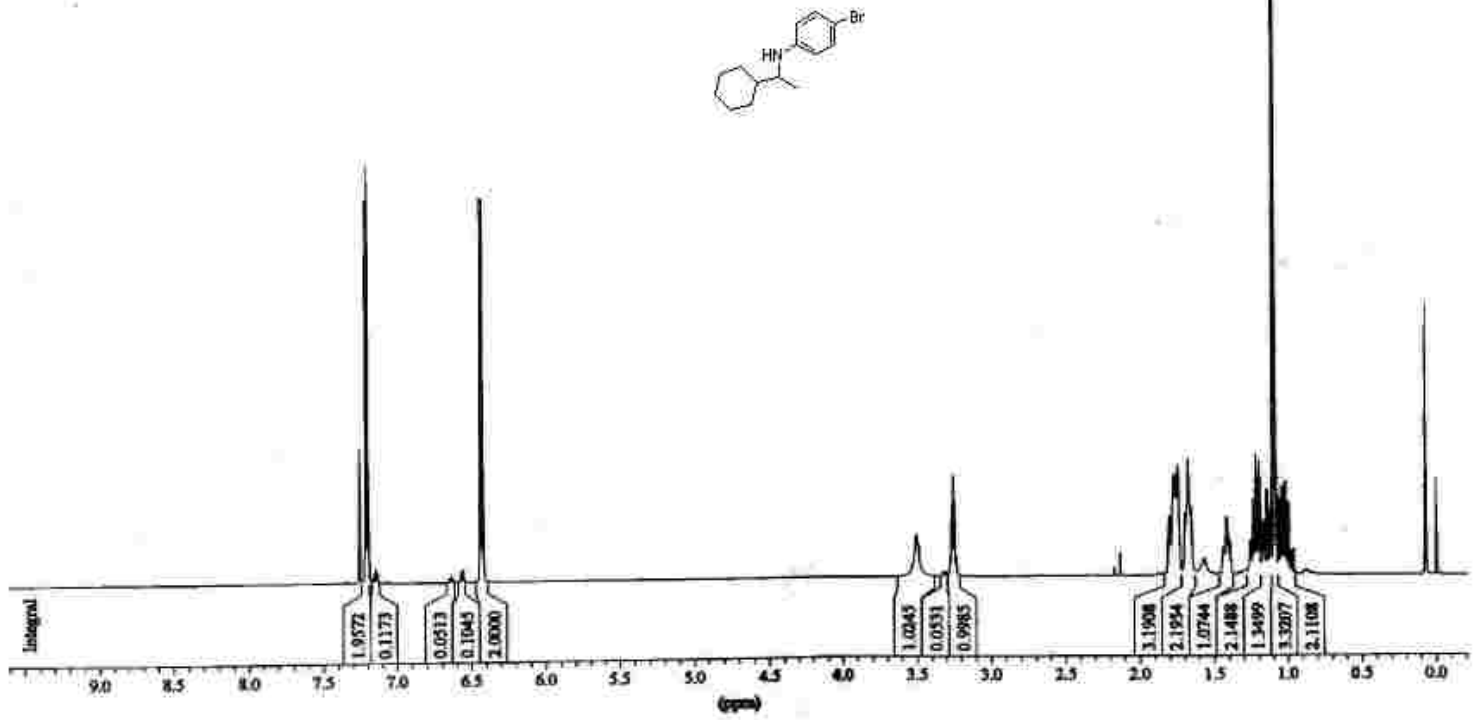

Bruker Avance 600 probe: $13 \mathrm{C}-1 \mathrm{H}$ DUIL

sample: wzy-12-1

solven: CDCL3 spectrum: $13 \mathrm{C}$

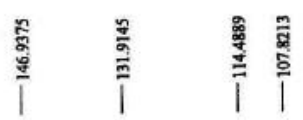

है।
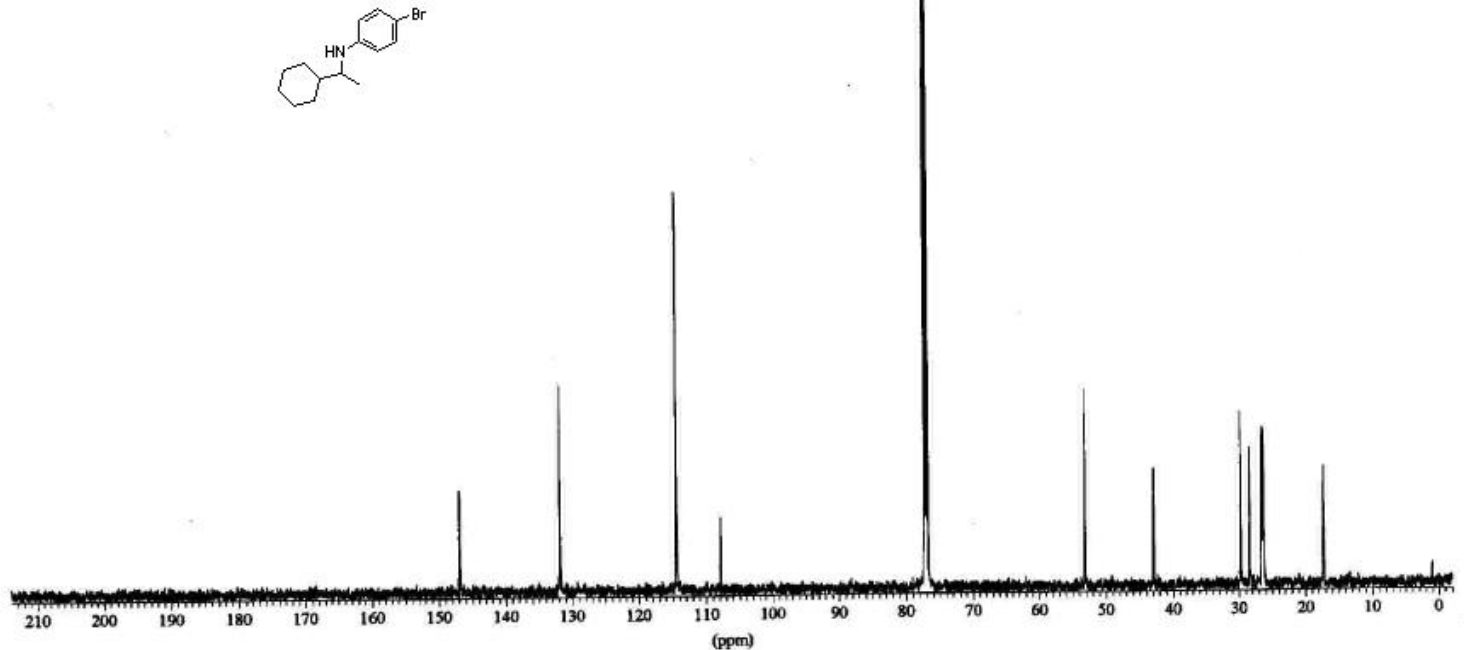
The NMR spectra of $\mathbf{6 p}$

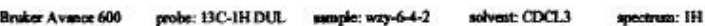

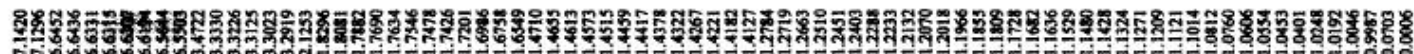
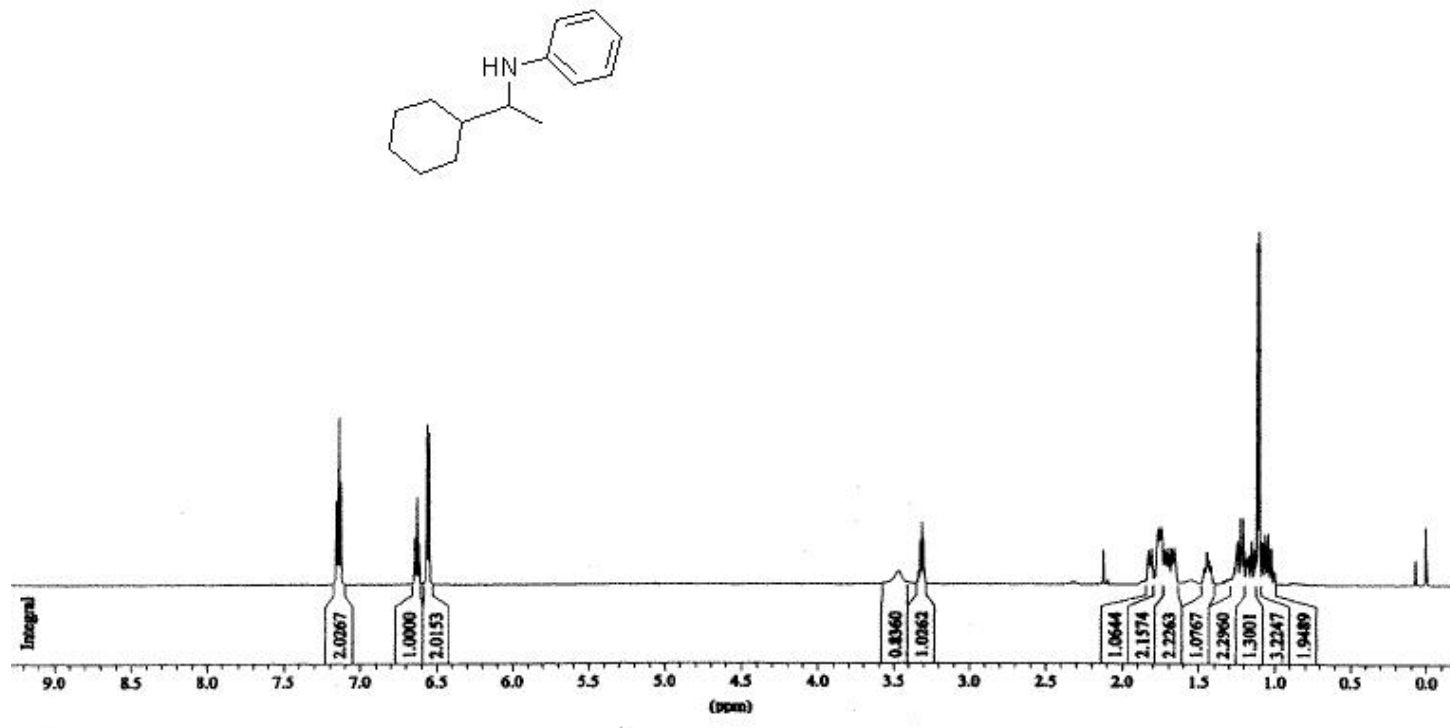
The NMR spectra of $\mathbf{6 q}$

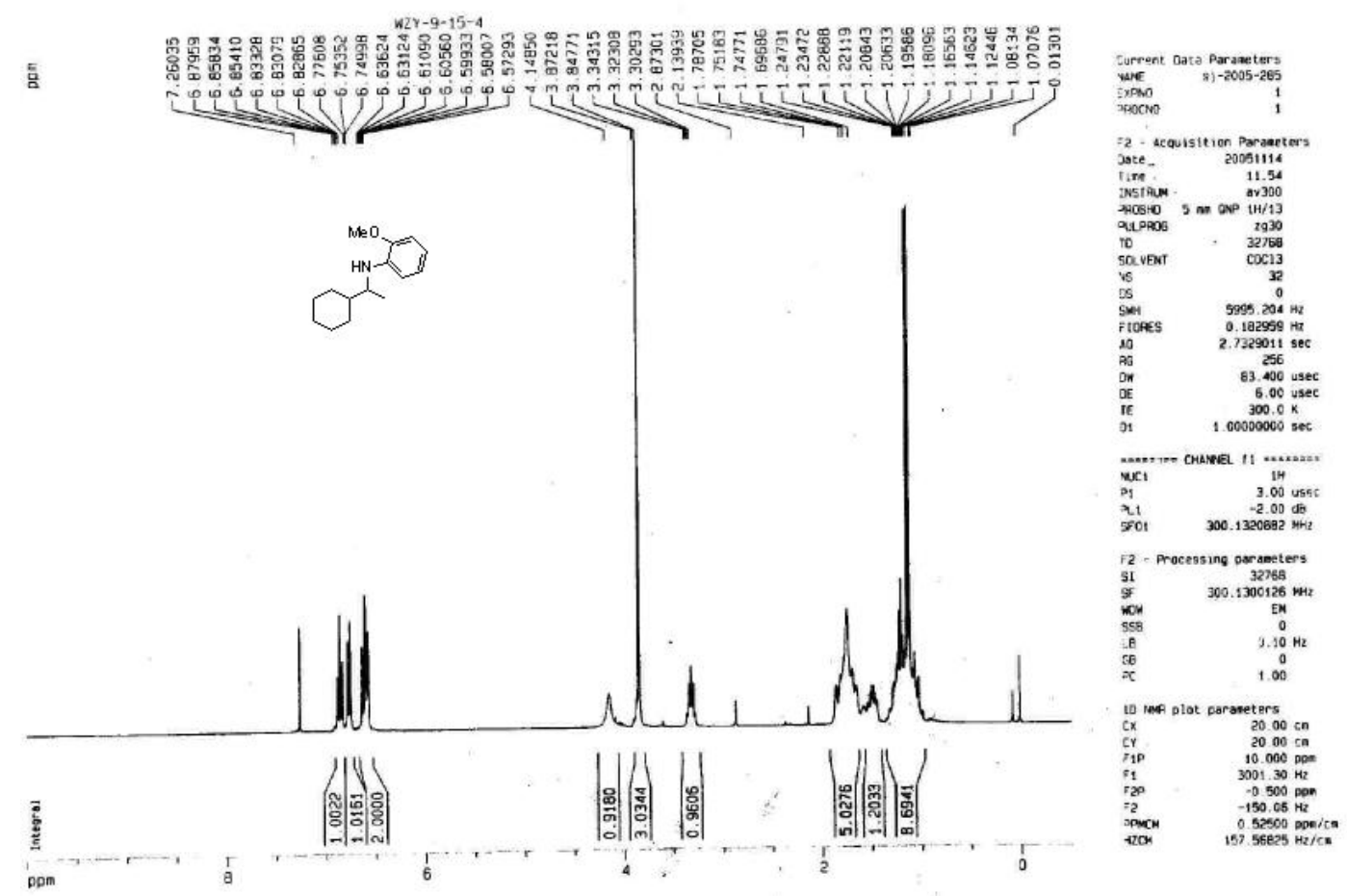

$w 2 \gamma=9-15-4$
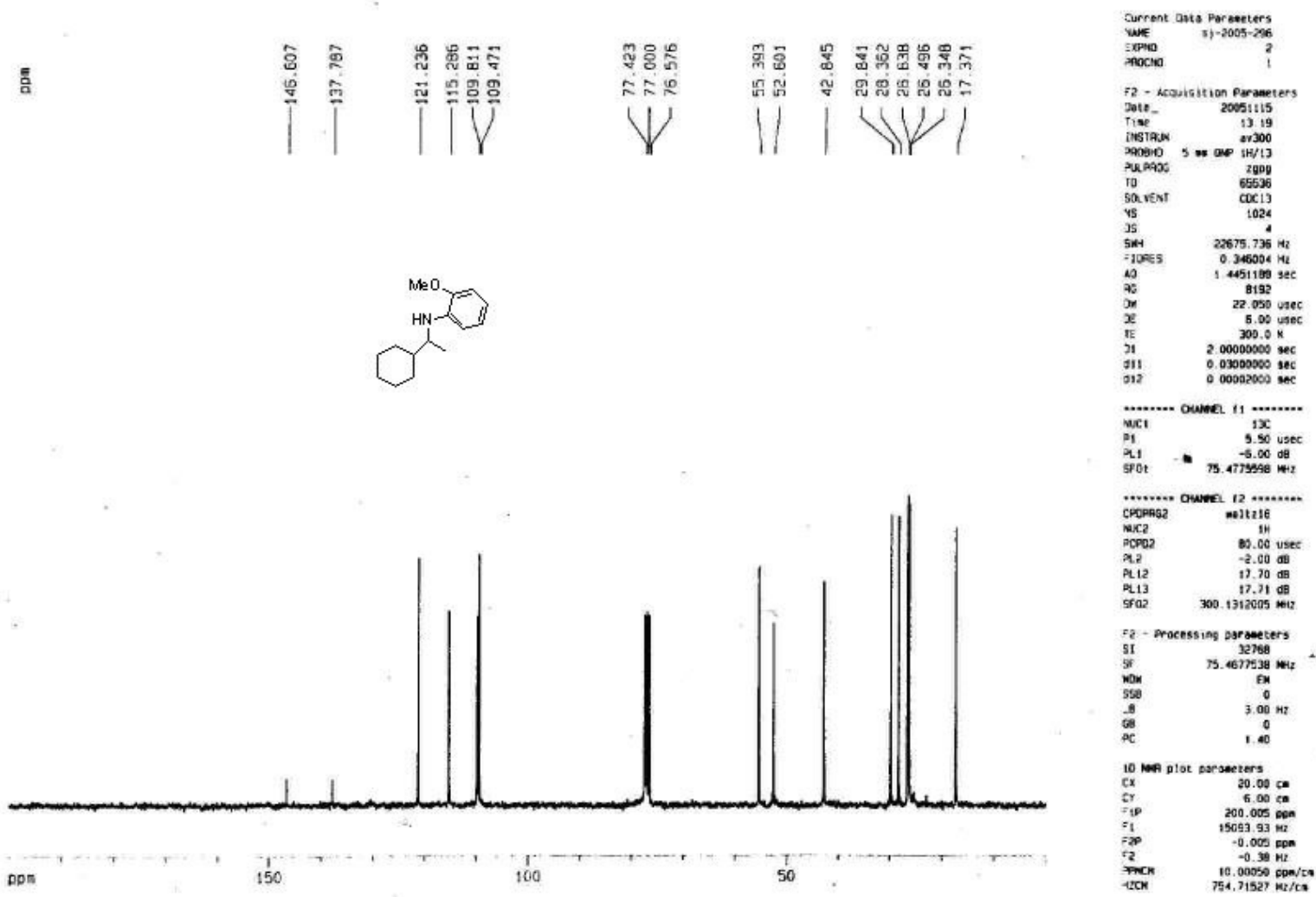
The NMR spectra of $\mathbf{6 r}$
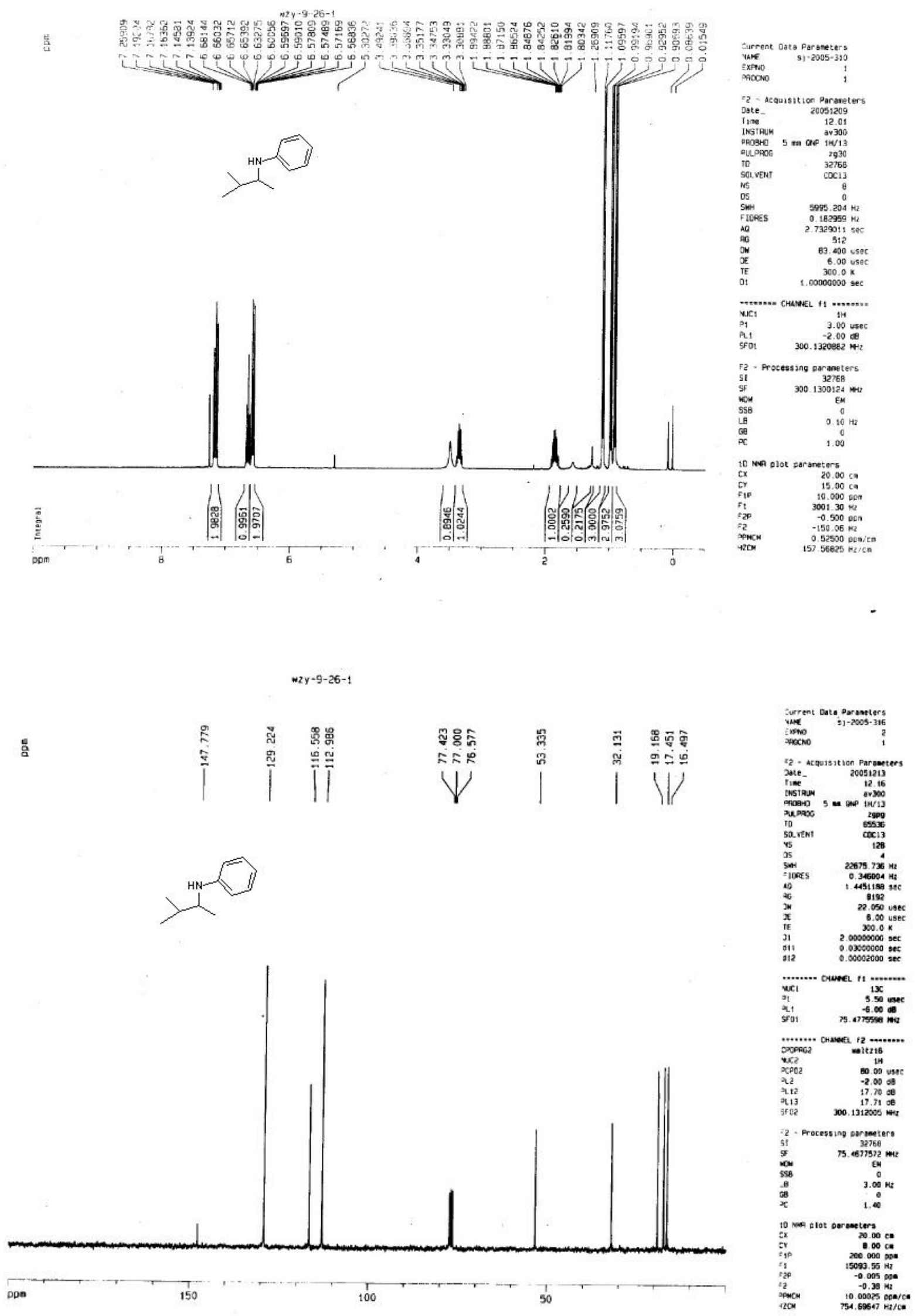
The NMR spectra of $\mathbf{6 s}$

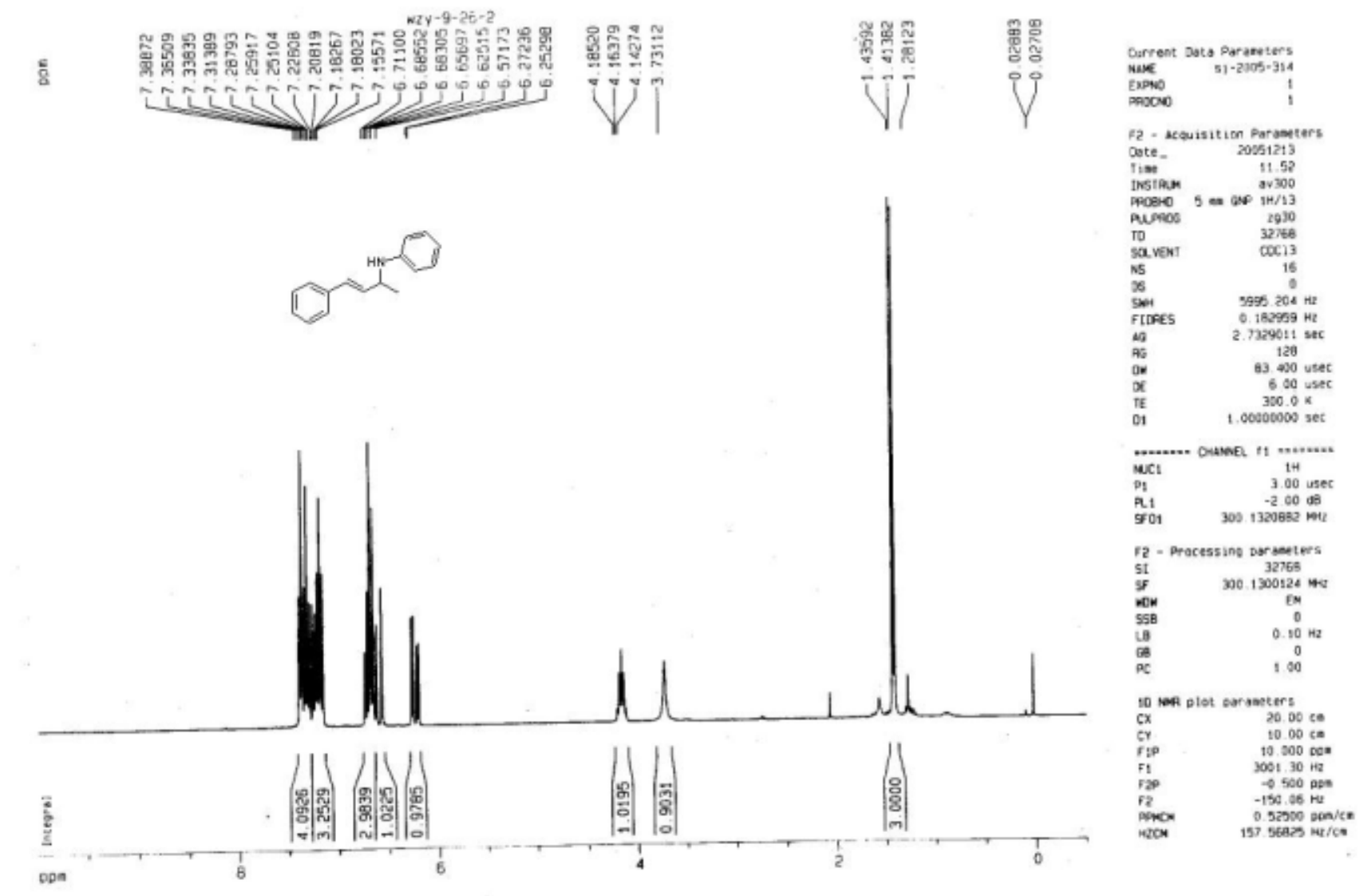


The HPLC spectra of $\mathbf{6 a}$

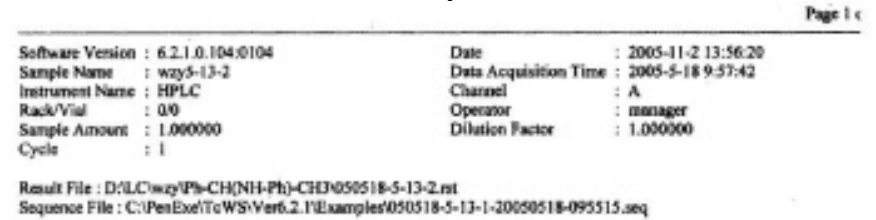

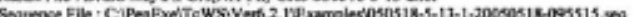

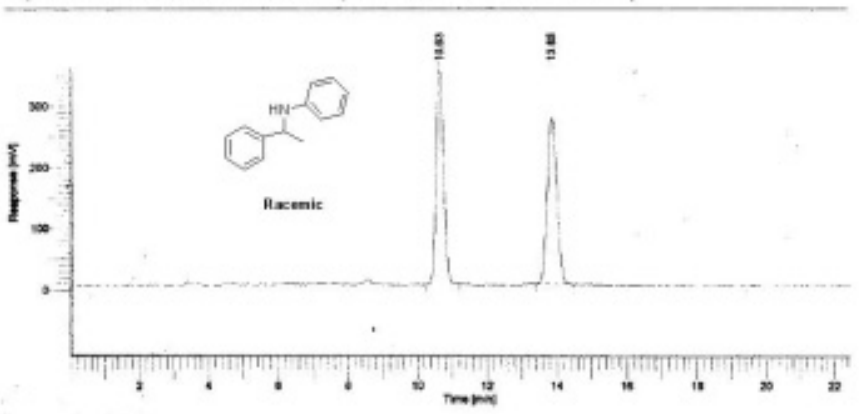

\section{DEFAULT REPORT}

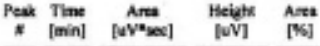

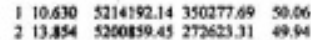

$$
\begin{aligned}
& \text { 10415051 58 } 622901.00100 .00
\end{aligned}
$$

Masing Compenem Repoet

Componeme Expected Rosention (Callitranion File)

PE serbeano

Software Versice : 0.2.1.0.10450104

Sample Name : was-30-

Instrimele

Sample Amourt : 1.000000

Cycle

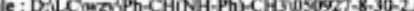

Sequence File : C.PRenExelTeWSiVer6.2.I Examplesi050927-8-30-2.5e9

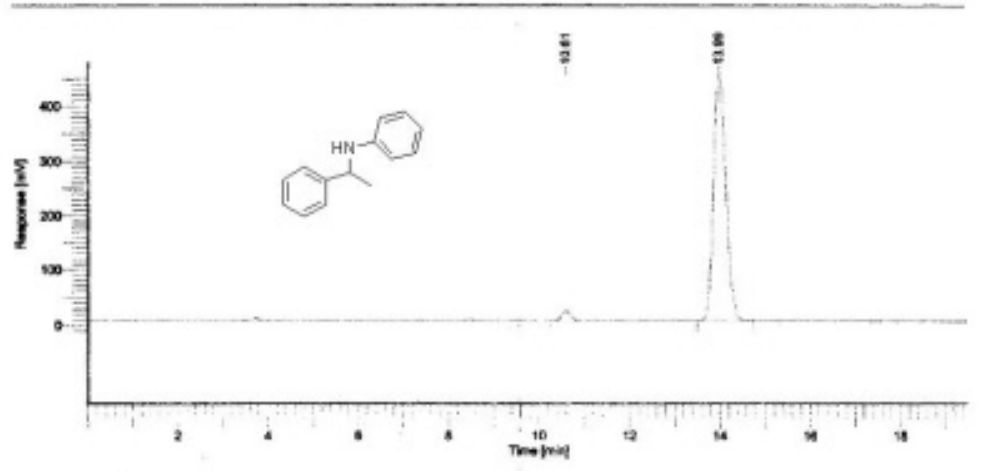

\section{DEFAULT REPORT}

Date : 2005-9.27 16:46:58

ivition Time : 2005-9-27 14:47:32

Channel : A
Operator : manager 
The HPLC spectra of $\mathbf{6 b}$

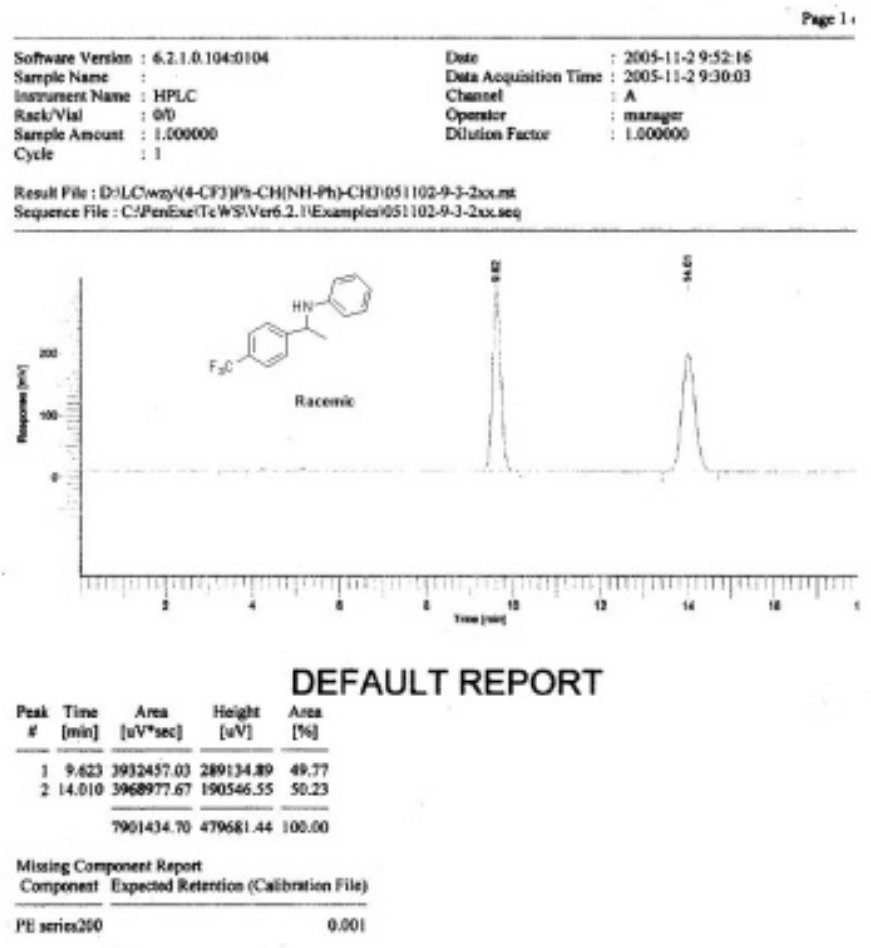

Page 1 of 1

\begin{tabular}{|c|c|c|c|}
\hline $\begin{array}{l}\text { Software Version } \\
\text { Sampie Name } \\
\text { Instrument Name } \\
\text { Raxif Vial } \\
\text { Sample Amount } \\
\text { Cyele }\end{array}$ & $\begin{array}{l}: 6.2 .0 .104: 0104 \\
: \text { way-2-2 } \\
: \text { HPLC } \\
: 000 \\
: 1.000000 \\
: 1\end{array}$ & $\begin{array}{l}\text { Dete } \\
\text { Desa Acquisition Time } \\
\text { Channel } \\
\text { Operstor } \\
\text { Dilution Facter }\end{array}$ & $\begin{array}{l}2005-11-2 \text { 10:-14:33 } \\
2005-11-29=53: 01 \\
\text { A } \\
\text { maneger } \\
1.000000\end{array}$ \\
\hline
\end{tabular}

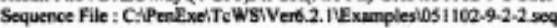

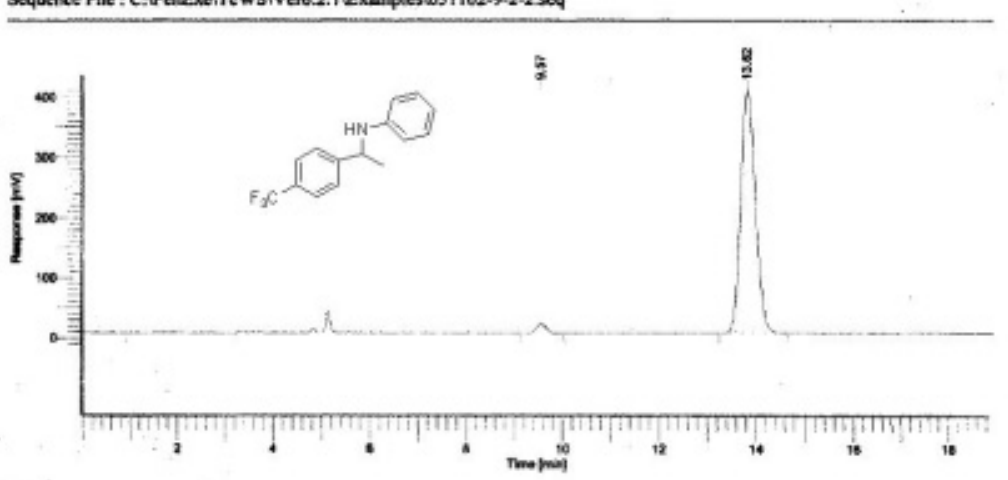

\section{DEFAULT REPORT}

$$
\begin{aligned}
& \begin{array}{l}
\text { Peak Time Area Height Area } \\
\text { [min] [uV*sec] } \\
{[\mathrm{uV}]}
\end{array} \\
& 19.566 \quad 195153.35 \quad 14758.24 \quad 2.26 \\
& \begin{array}{rrrr}
213.821 & 195153.35 & 14758.24 & 2.26 \\
& 457109.52 & 402504.16 & 97.74
\end{array} \\
& 8657256.87 \overline{417262.40} \overline{100.00}
\end{aligned}
$$


The HPLC spectra of $\mathbf{6 c}$

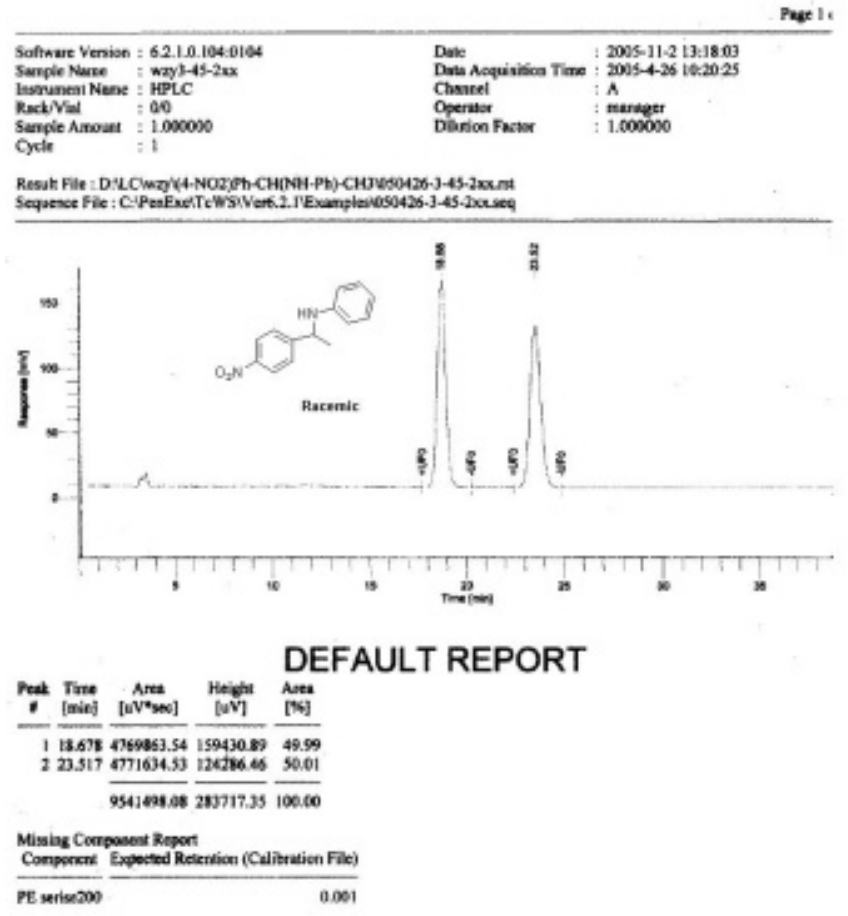

\begin{tabular}{|c|c|c|c|}
\hline $\begin{array}{l}\text { Sofluare Versicen } \\
\text { Sample Nerne. }\end{array}$ & $\begin{array}{l}6.21 .0 .104: 0104 \\
6203-33-3\end{array}$ & $\begin{array}{l}\text { Date } \\
\text { Dath Acquisiticen Time }\end{array}$ & $\begin{array}{l}=2005-10-818 \times 16: 00 \\
2005-108: 17: 45: 22\end{array}$ \\
\hline $\begin{array}{l}\text { Sampie Nintas } \\
\text { lnstrument Name }\end{array}$ & $\begin{array}{l}\text { HPLC } \\
\text { HPLO-5 }\end{array}$ & Charnel & \\
\hline Rack/Vial & $: 00$ & Oserator & manager \\
\hline Sampit Amorest & $: 1,000000$ & Dilution Factose & $: 1.000000$ \\
\hline Cycle & $: 1$ & & \\
\hline
\end{tabular}

Cycle

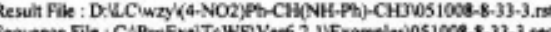

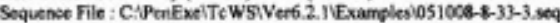

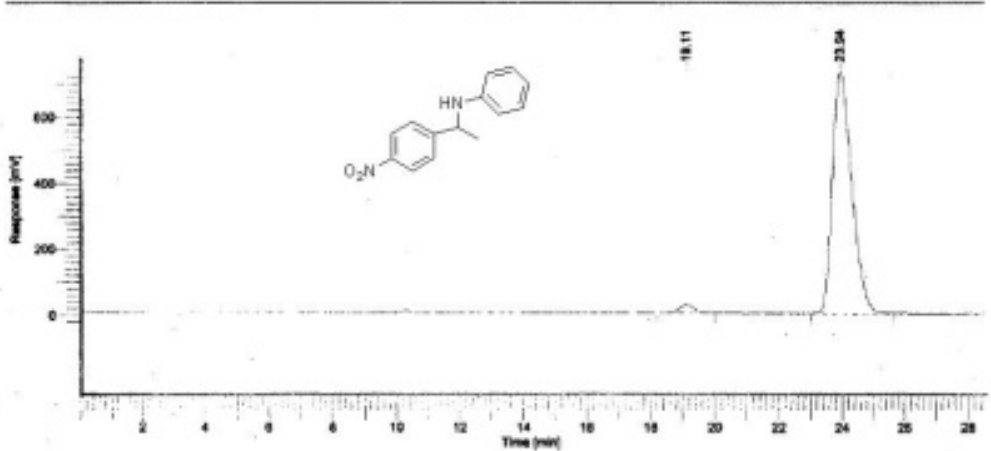

\section{DEFAULT REPORT}

\begin{tabular}{|c|c|c|c|}
\hline $\begin{array}{l}\text { Peak Time } \\
\text { [min] }\end{array}$ & $\begin{array}{l}\text { Aren } \\
{\left[\mathrm{w}^{*} \operatorname{sen}\right]}\end{array}$ & $\begin{array}{l}\text { Height } \\
\text { [uV] }\end{array}$ & $\begin{array}{l}\text { Area } \\
\text { [\$] }\end{array}$ \\
\hline \multirow{3}{*}{$\begin{array}{ll}1 & 19.111 \\
2 & 23.937\end{array}$} & $\begin{array}{r}794060.20 \\
1906930.77\end{array}$ & 26465.76 & 2.49 \\
\hline & & & \\
\hline & 31863449.47 & 754200.14 & 106.00 \\
\hline \multicolumn{4}{|c|}{ Mlssing Component Report } \\
\hline serise 200 & & & 0.001 \\
\hline
\end{tabular}


The HPLC spectra of $\mathbf{6 d}$

\begin{tabular}{|c|c|c|c|}
\hline 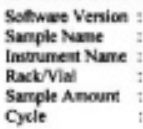 & 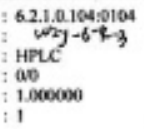 & 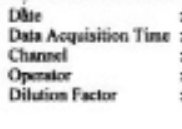 & 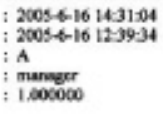 \\
\hline
\end{tabular}

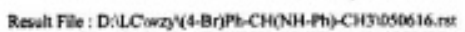

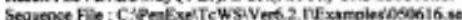

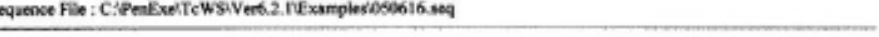

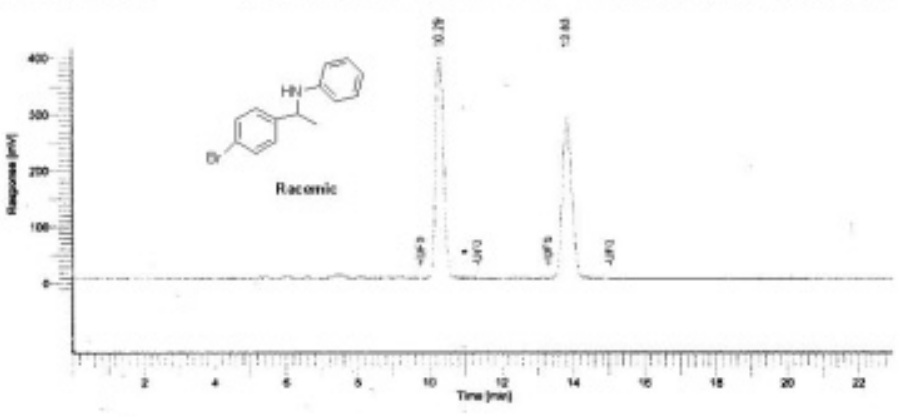

DEFAULT REPORT

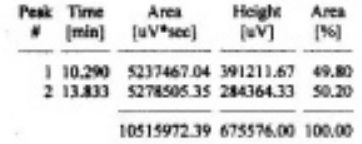

Missing Componens Report
Component Expected Retention (Calitorstion File)

\begin{tabular}{|c|c|c|c|}
\hline & & \\
\hline $\begin{array}{l}\text { Software Venien } \\
\text { Sanple Neme } \\
\text { Instrument Nume } \\
\text { Rack'Vial } \\
\text { Sample Amount } \\
\text { Cycle }\end{array}$ & $\begin{array}{l}\text { 6.2.1.0.104:0104 } \\
\text { w.2.3.-33-2 } \\
\text { HFLC } \\
00 \\
1.600000 \\
1\end{array}$ & $\begin{array}{l}\text { Date } \\
\text { Date Acquisition Tine } \\
\text { Chennel } \\
\text { Operntor } \\
\text { Dilucion Facter }\end{array}$ & $\begin{array}{l}2005-10-8 \text { 16:40:08 } \\
2005-10-8 \text { 16:18:42 } \\
A \\
\text { manager } \\
1.000000\end{array}$ \\
\hline
\end{tabular}

PE seriseaso 0.001

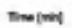

Result File : D:UClwayk4-Br) Ph-CH(NH-Pa) CHaves 1008-8-33-2 mt

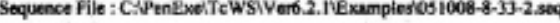

\begin{tabular}{|c|c|c|c|c|}
\hline Pout & Time & $\begin{array}{c}\text { Area } \\
{\left[\mathrm{uV}^{*} \sec \right]}\end{array}$ & $\begin{array}{l}\text { Height } \\
\text { [uV] }\end{array}$ & $\begin{array}{l}\text { Ares } \\
{[\%]}\end{array}$ \\
\hline & $\begin{array}{l}10.92 \\
14,444\end{array}$ & $\begin{array}{r}71188.40 \\
29073831.85\end{array}$ & $\begin{array}{l}53336.11 \\
1.260+06\end{array}$ & $\begin{array}{r}2.58 \\
97.12\end{array}$ \\
\hline 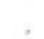 & & 29445020.26 & $1.310+06$ & 100.00 \\
\hline $\begin{array}{l}\text { Mese } \\
\text { Com }\end{array}$ & ing Coent & $\begin{array}{l}\text { ponent Report } \\
\text { Eupested Ret }\end{array}$ & Itntion (C. & Iilbration File) \\
\hline
\end{tabular}

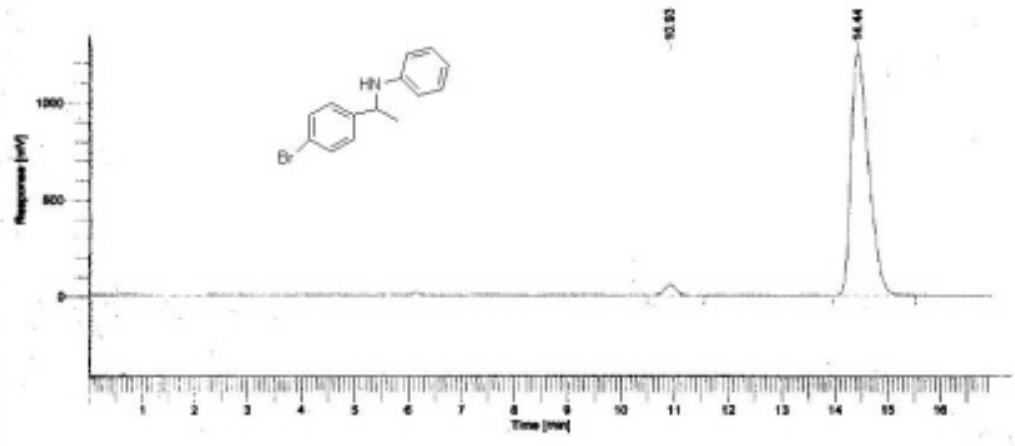

\section{DEFAULT REPORT}


The HPLC spectra of $\mathbf{6 e}$

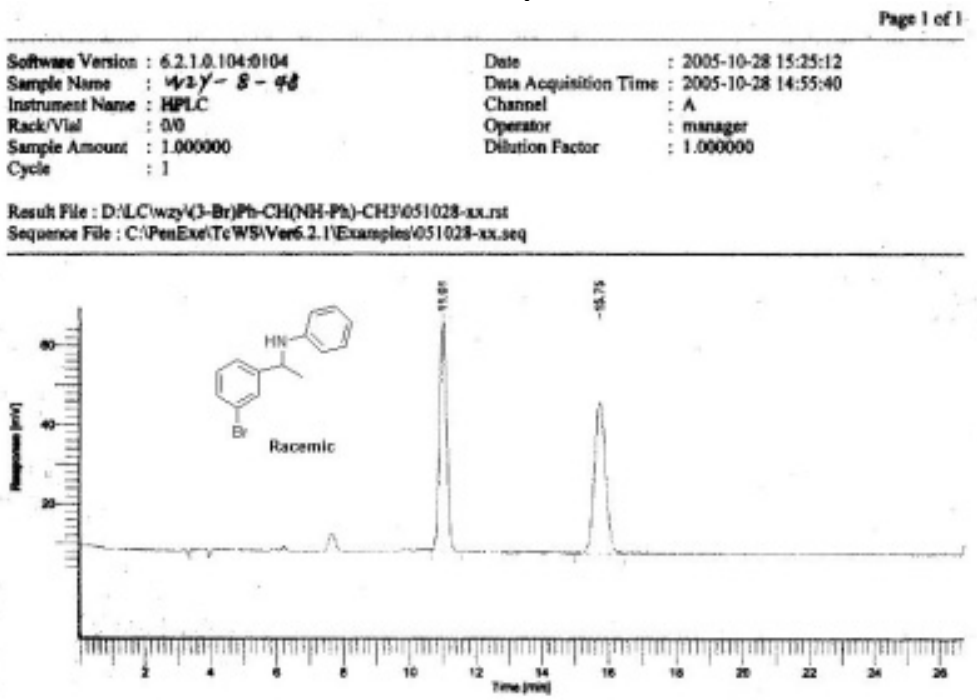

\section{DEFAULT REPORT}

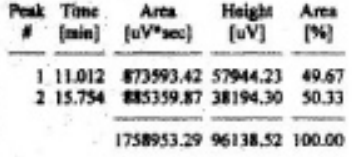

Mising Component Report
Compones Expectod Retention (Culturnion File)

\begin{tabular}{|c|c|c|c|c|}
\hline 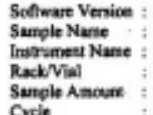 & $\begin{array}{l}6.2 .1 .0 .194 .004 \\
\text { ways.1-3 } \\
\text { HPLC } \\
00 \\
1.000000 \\
1\end{array}$ & 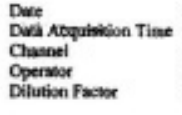 & 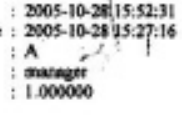 & ; \\
\hline
\end{tabular}

PE $\approx$ eriena00 0.001

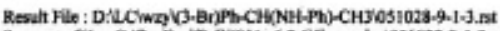

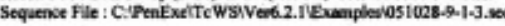

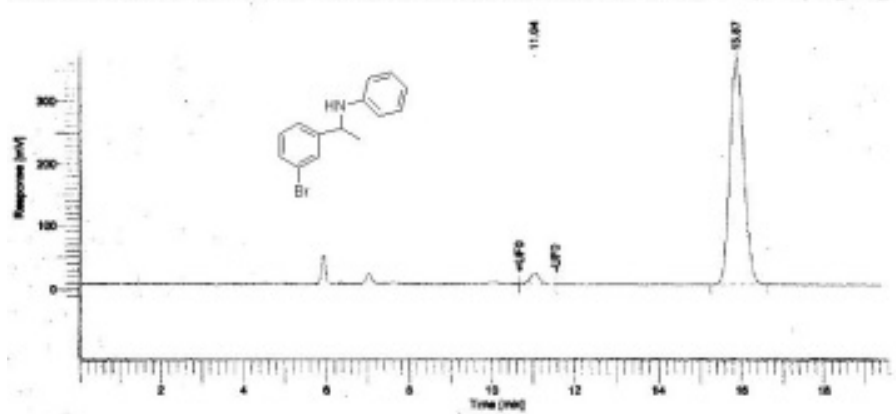

\section{DEFAULT REPORT}

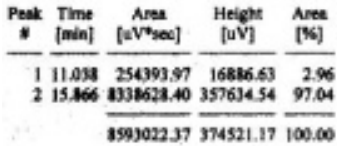

Missing Conponect Report
Consponsat Expostid Retention (Caliteation File)

$\overline{\text { PE serimav0 }} 0.001$ 
The HPLC spectra of $\mathbf{6 f}$

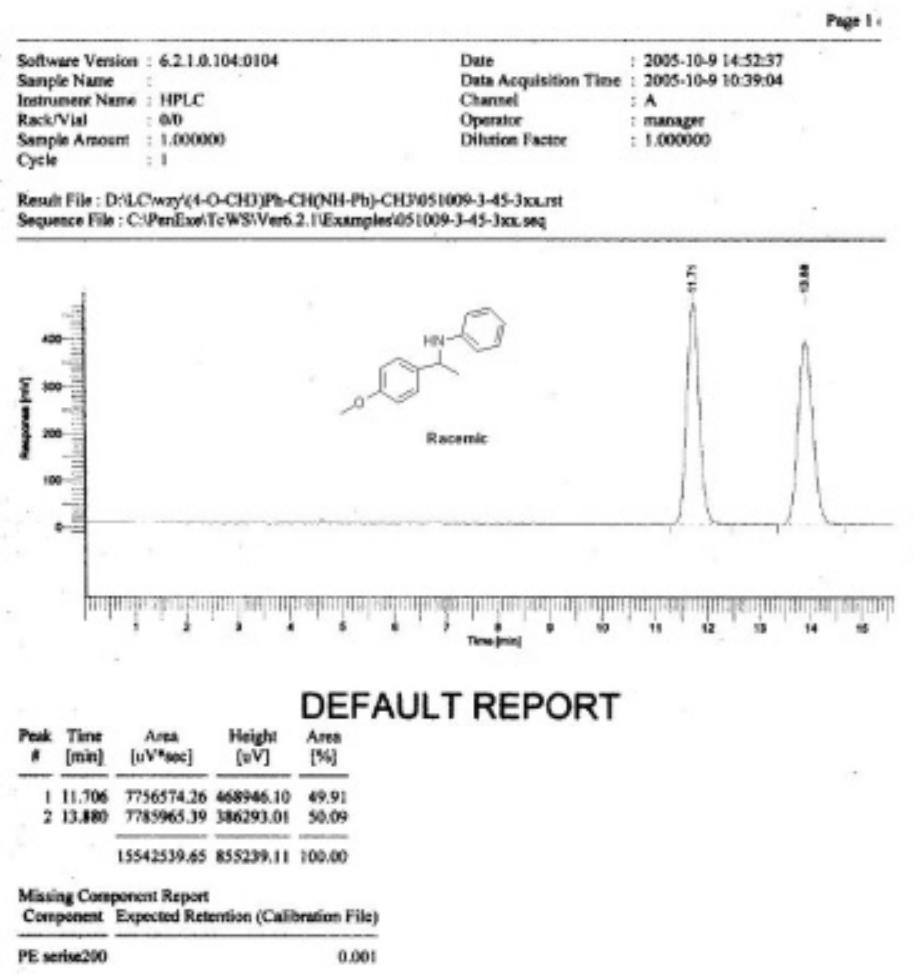

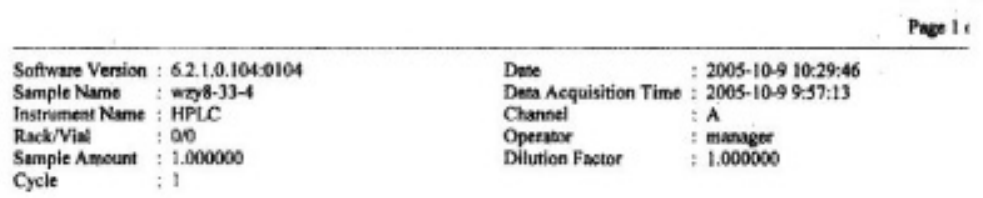

Result File : D:LCaray(4-0-CH3)Ph-CH(NH-Ph)-CH3v051009-8.33-4.rst Sequnte File:COP.

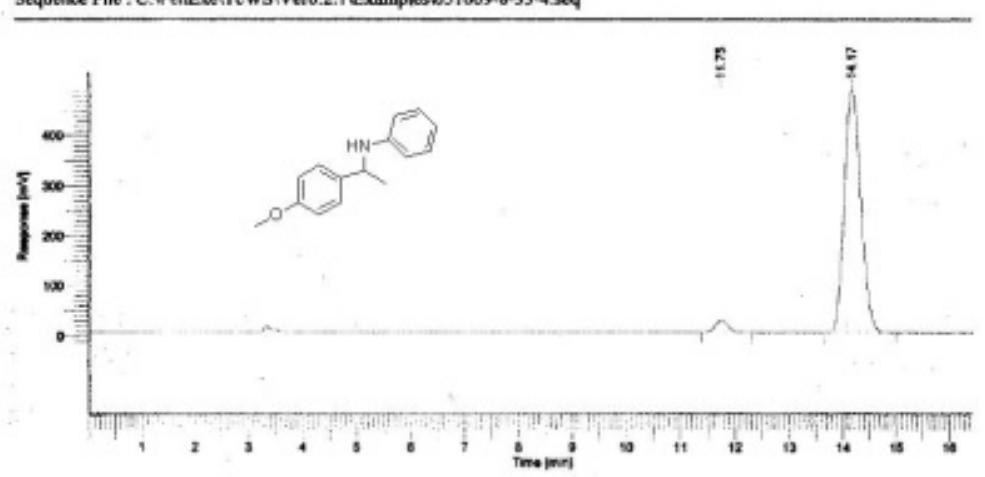

\section{DEFAULT REPORT}

\begin{tabular}{|c|c|c|c|c|}
\hline Penk & $\begin{array}{l}\text { Time } \\
\text { [min] }\end{array}$ & $\begin{array}{c}\text { Arca } \\
{\left[\mathrm{uV}^{*} \mathrm{sec}\right]}\end{array}$ & $\begin{array}{l}\text { Height } \\
\text { [uV] }\end{array}$ & $\begin{array}{l}\text { Area } \\
\text { [\%] }\end{array}$ \\
\hline & $\begin{array}{l}11.750 \\
14.17\end{array}$ & $\begin{array}{r}405683.79 \\
10418734.07\end{array}$ & $\begin{array}{r}24005.72 \\
483893.22\end{array}$ & $\begin{array}{r}3.77 \\
96.23\end{array}$ \\
\hline & & $10 \times 27417.85$ & 507898.94 & 100.00 \\
\hline \multicolumn{4}{|c|}{$\begin{array}{l}\text { Missing Component Report } \\
\text { Compone Frnected Retention /Calibration File }\end{array}$} & oration File \\
\hline
\end{tabular}


The HPLC spectra of $\mathbf{6 g}$

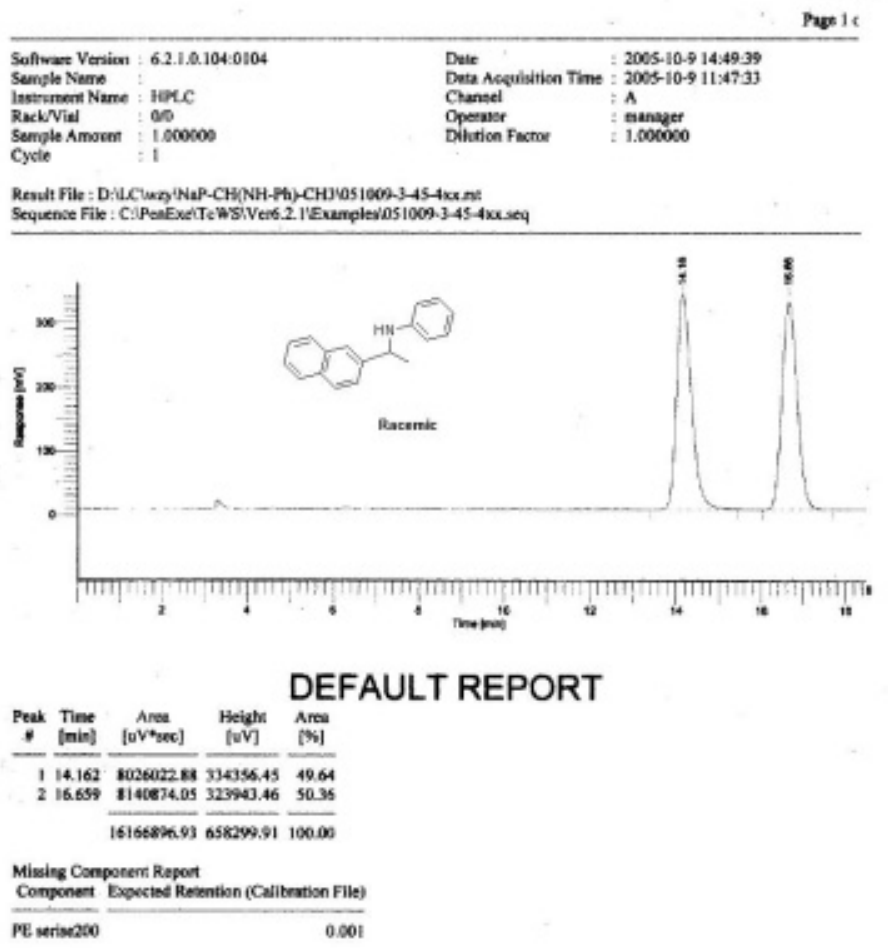

Page 1

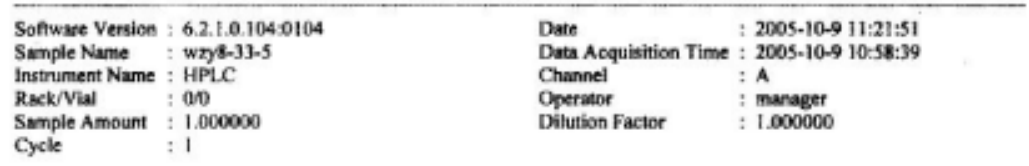

Result Pile : D:ULClwzy1NaP-CH(NH-Ph)-CHJ 051009-8-33-5.rst

Sequence File : C:PenExeiTcWSiVert.2. I Exampiesios 1009-8-33-5 seo

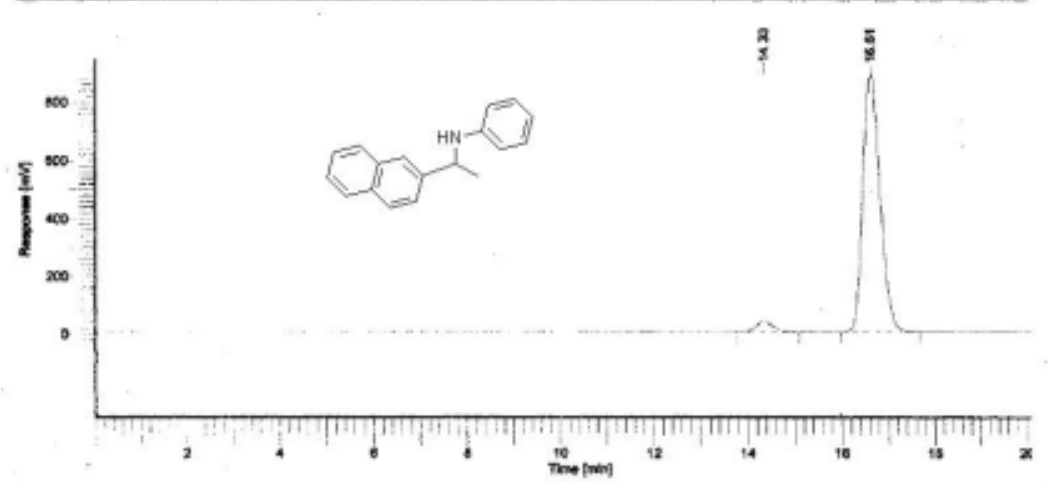

\section{DEFAULT REPORT}

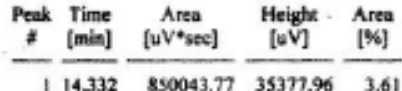

$$
\begin{aligned}
& \begin{array}{lllll}
14.332 & 850043.77 & 3597.96 & 3.61 \\
1 & 16.606 & 22590801.86 & 889345.58 & 96.39
\end{array} \\
& \text { 23560845.63 } 925223.54100 .00
\end{aligned}
$$

Missing Component Report

Component Expected Retention (Calibration File)

PE serise200 
The HPLC spectra of $\mathbf{6 h}$

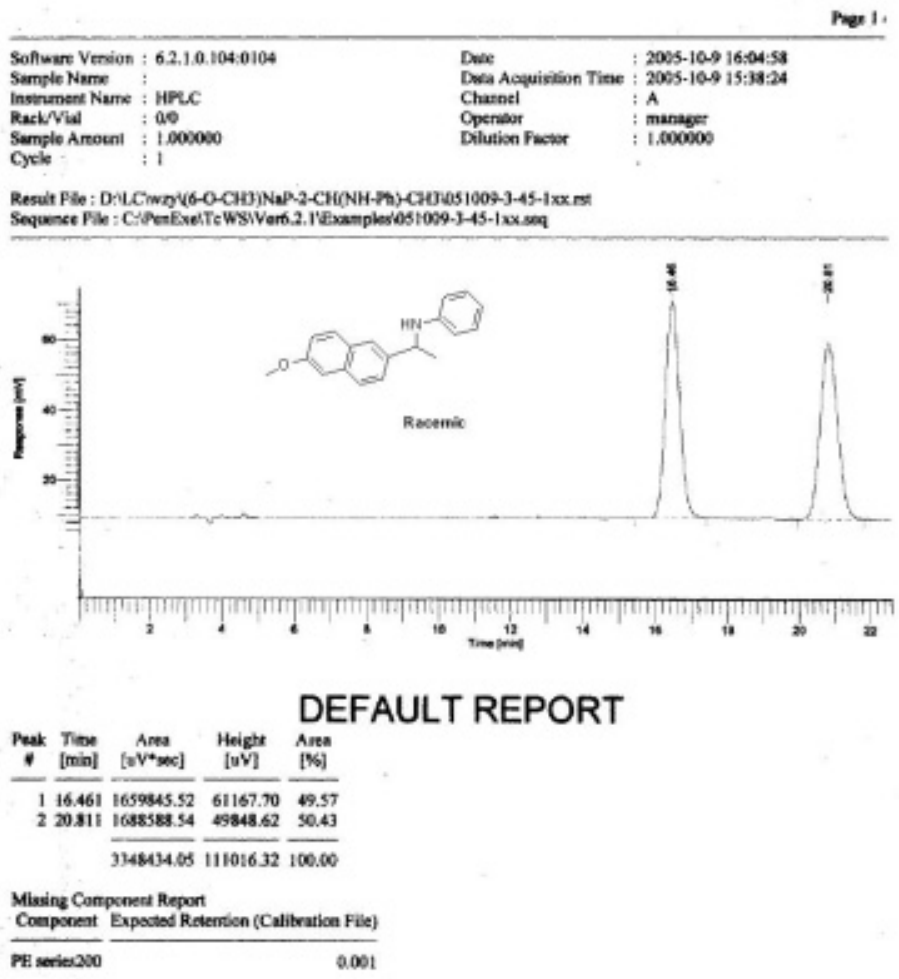

Page If

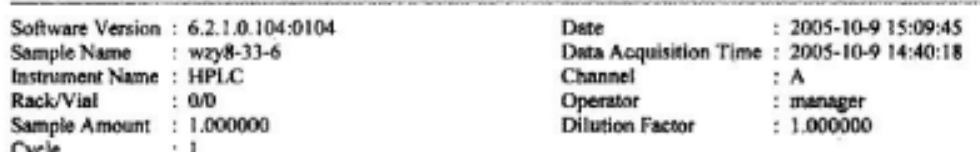

Sample Amount : 1.000000 Dilution Factor

Cyele : : 1

Result File : D L C Charg) (6-0-CH3) NaP-2-CH(NH-Ph)-CH31051009-8-33-6.rst

Seguence File : C:IPenExeiTcWSiVer6.2.1UExamaples $1051009-8-33-6.969$

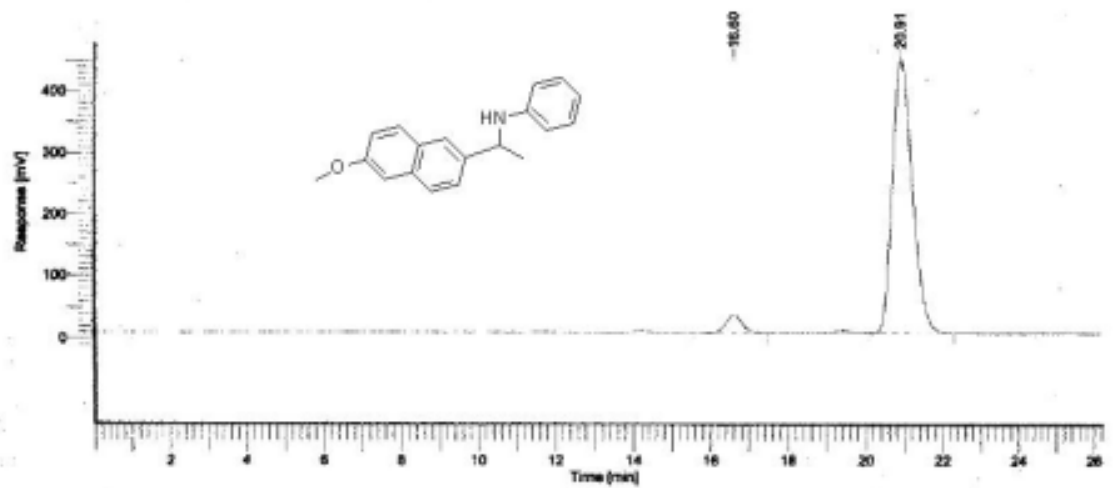

DEFAULT REPORT

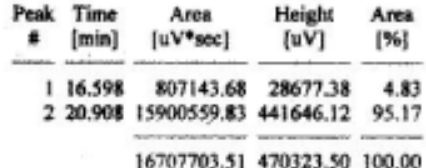

Missing Component Report

Component Expected Retention (Culibration File)

PE scries 200

0.001 
The HPLC spectra of $\mathbf{6 i}$

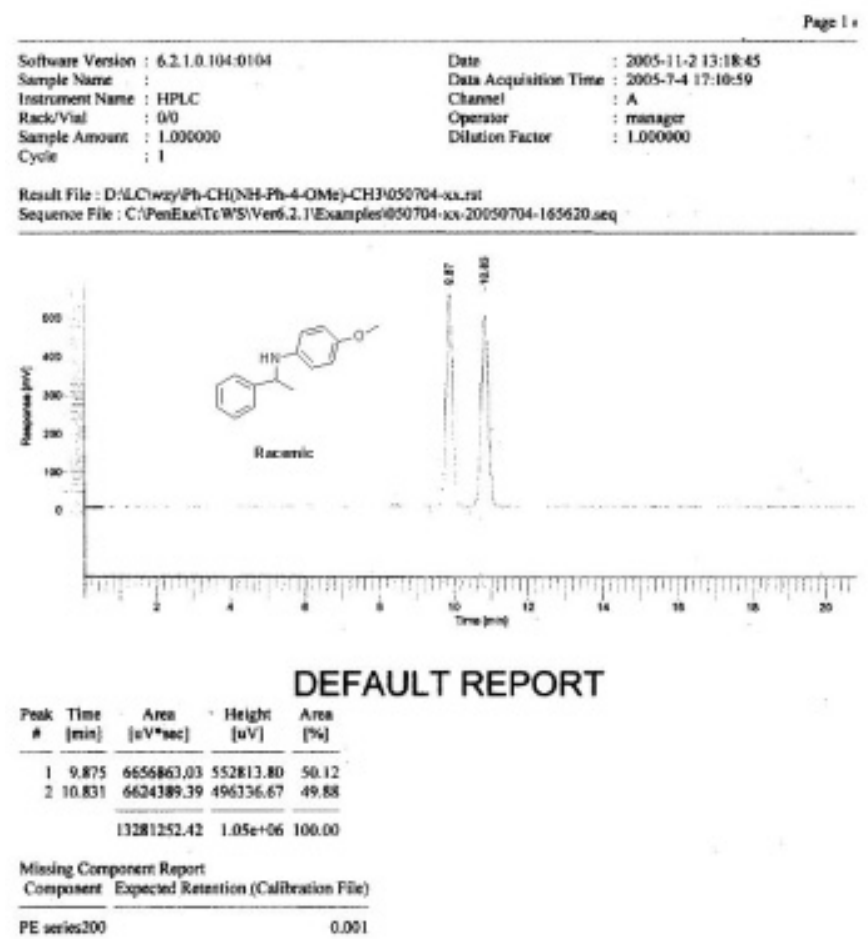

Pape Ic

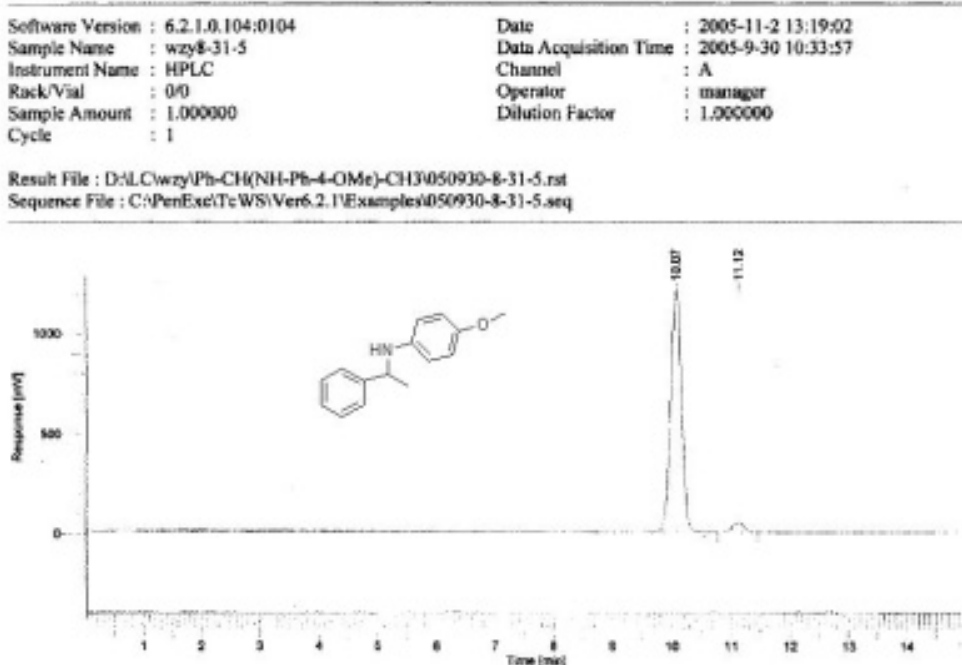

\section{DEFAULT REPORT}

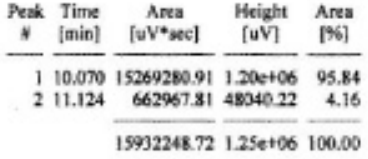

Missing Component Report

Component Expected Retentien (Calibration File)

$\mathrm{PE}$ series2000 
The HPLC spectra of $\mathbf{6 j}$

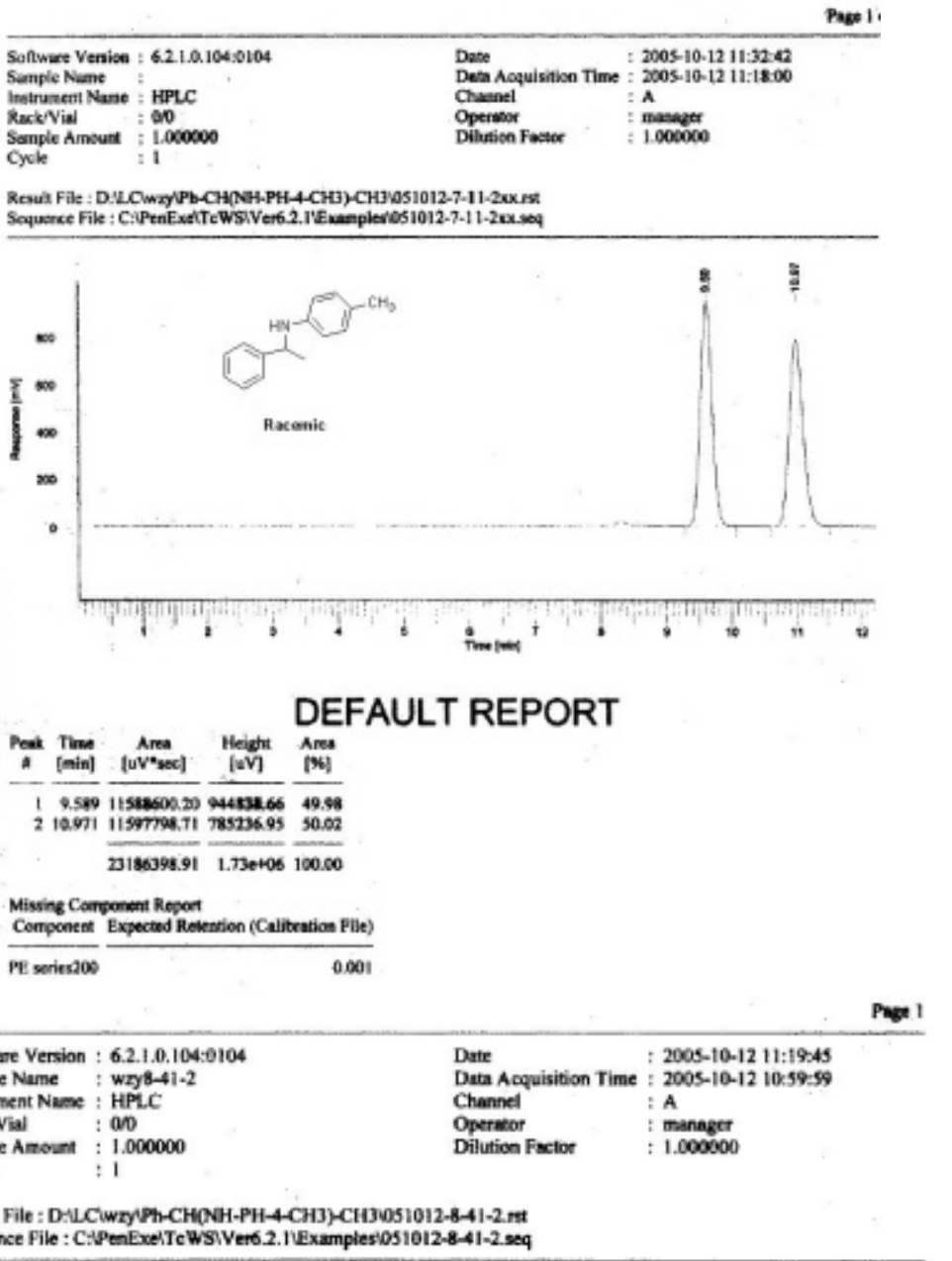

Sequence File : C:PenExelTcWsiVer6.2.1Examplesios1012-8-41-2.seq

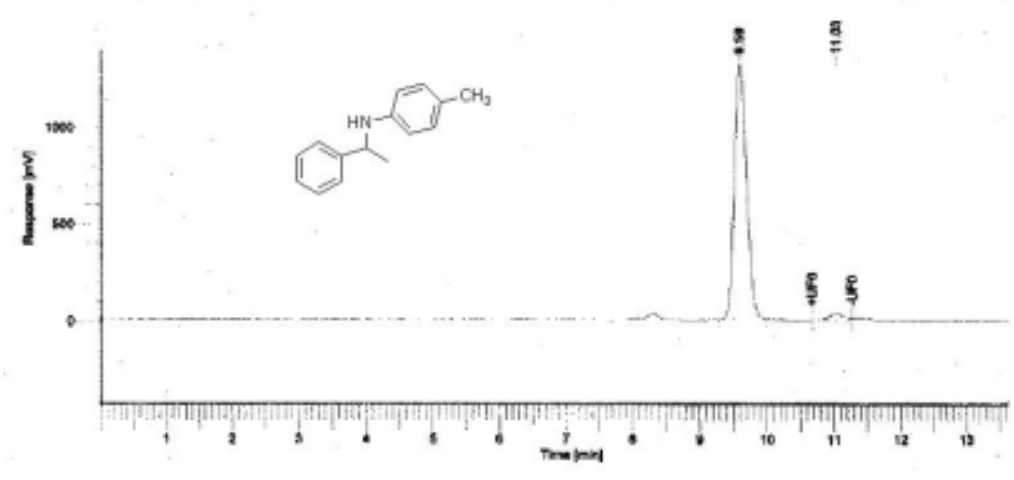

\section{DEFAULT REPORT}

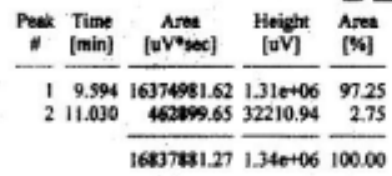

Missing Component Report

Compoment Expected Retention (Calioration File)

PE series200 
The HPLC spectra of $\mathbf{6 k}$

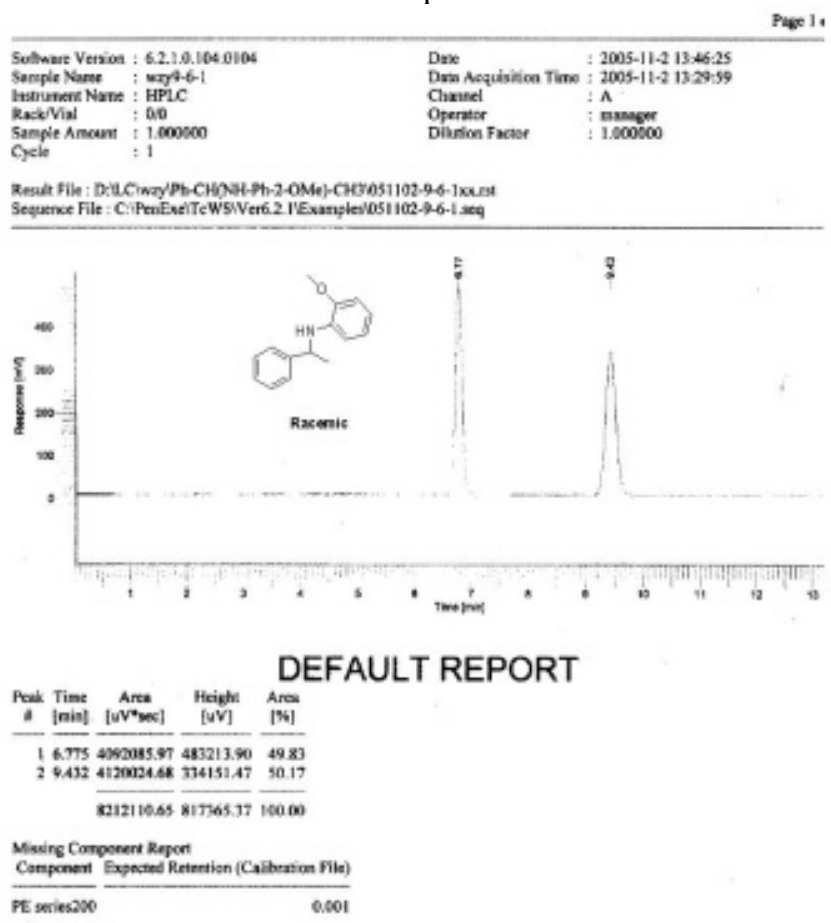

Page 16,

\begin{tabular}{|c|c|c|c|}
\hline Sottware Version & : $62.1 .0,104: 0104$ & Date & $: 2005-11-2$ 14;00:02 \\
\hline Sample Name & wrys-7.2 & Data Acquisition Time & $2005-11-2$ 13:47:16 \\
\hline Instrument Nave & : HPLC & Channel & $: A$ \\
\hline Rask/Vial & $: 60$ & Operator & : marager \\
\hline Sample Amouns: & $: 1000000$ & Dilution Faxtor & $=1.000000$ \\
\hline Cycio & $: 1$ & & \\
\hline
\end{tabular}

Cycis

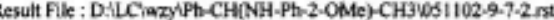
Sequence File : C.PenExeiTeWSiVer6.2.11Examplesi0s1 102-9.7-2. seq

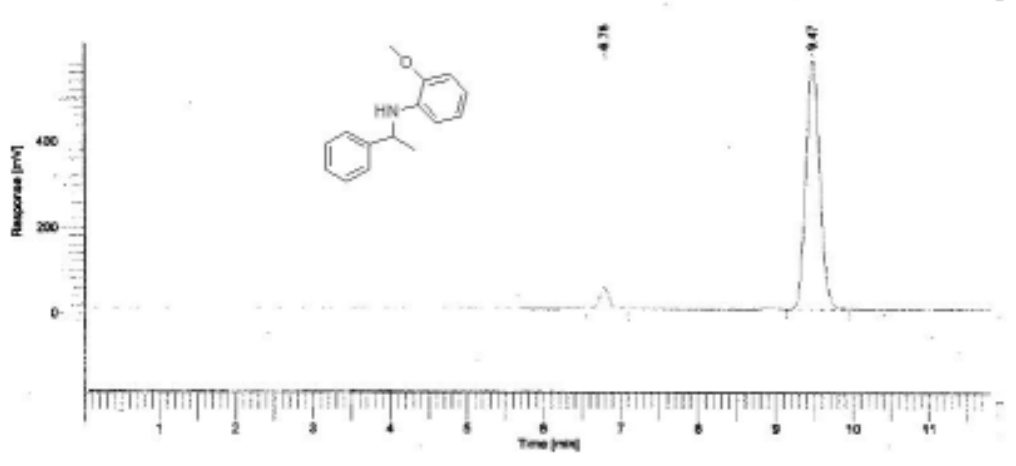

\section{DEFAULT REPORT}

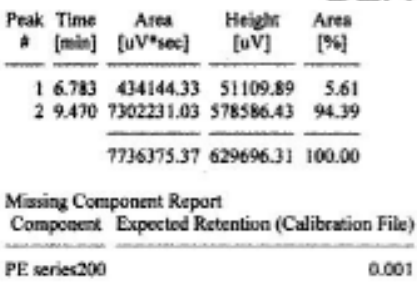


The HPLC spectra of $\mathbf{6 l}$

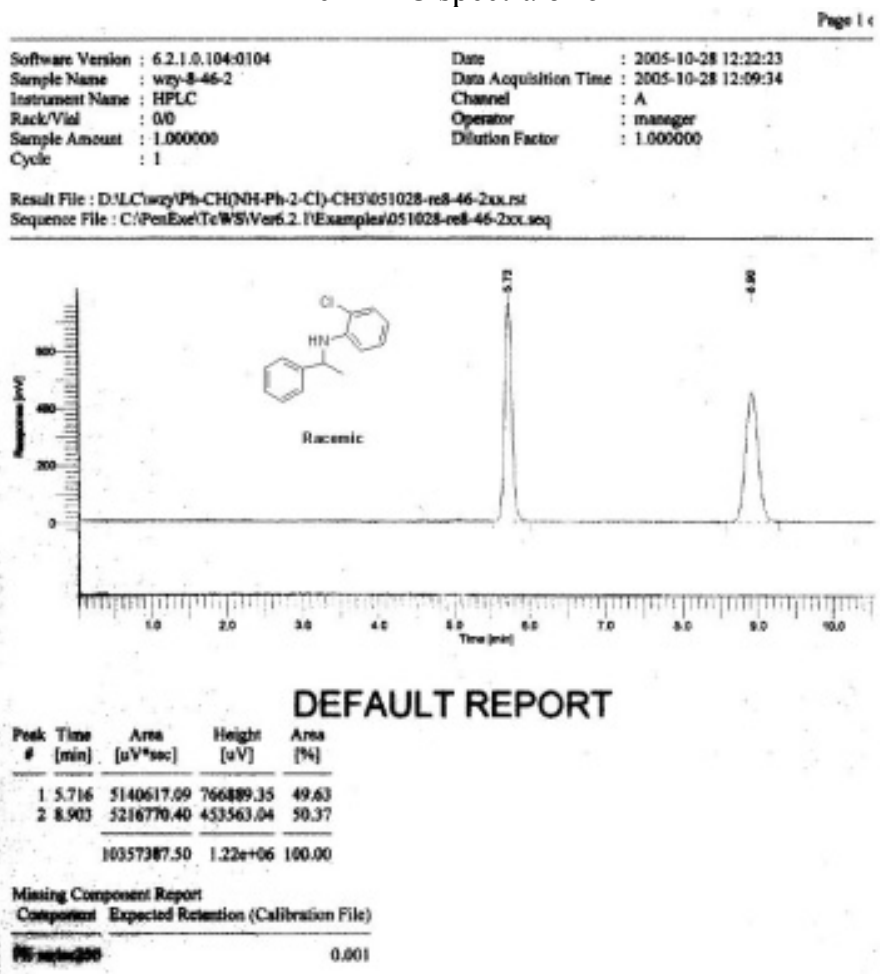

and

\begin{tabular}{|c|c|c|c|}
\hline $\begin{array}{l}\text { Soffware Vernion } \\
\text { Sample Nene } \\
\text { Instrument Nane } \\
\text { Rask/Vial } \\
\text { Sample Anoumt } \\
\text { Cycle }\end{array}$ & $\begin{array}{l}: 6.2 .1 .0 .104: 0104 \\
: \text { W.2y9-1.2 } \\
: \text { HPLC } \\
: 000 \\
: 1.000000 \\
: 1\end{array}$ & $\begin{array}{l}\text { Date } \\
\text { Deta Aoquisition Time } \\
\text { Chaneel } \\
\text { Operator } \\
\text { Dilution Factor }\end{array}$ & $\begin{array}{l}: 2005-10-2814: 21: 14 \\
: 2005 \cdot 10-28 \text { 12:23:59 } \\
: A \\
: \text { marager } \\
: 1.000000\end{array}$ \\
\hline
\end{tabular}

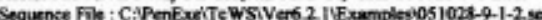

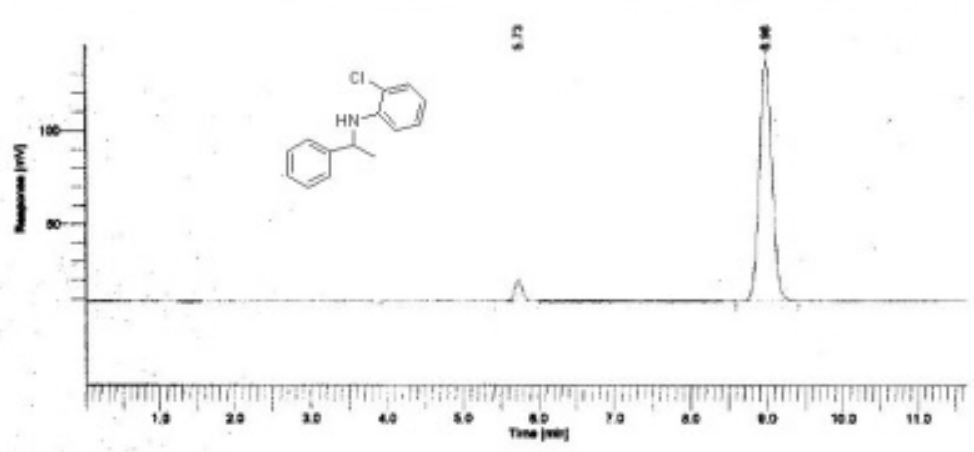

\section{DEFAULT REPORT}

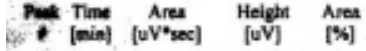

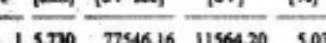

$$
\begin{aligned}
& \begin{array}{rrrr}
1.5 .790 & 77546.16 & 11564.20 & 5.03 \\
2.975 & 1463554.29 & 127582.76 & 94.97
\end{array} \\
& \overline{1541100.44} \overline{139146.96} \overline{100.00}
\end{aligned}
$$

Missing Compoeent Repert

Componeat Exported Retentios (Calibration File)

PE aries200 
The HPLC spectra of $\mathbf{6 m}$

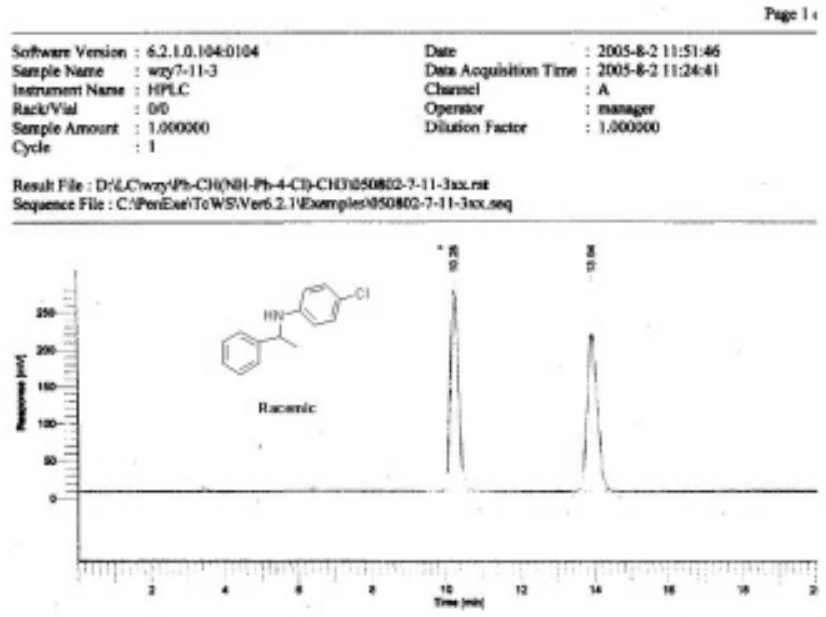

DEFAULT REPORT

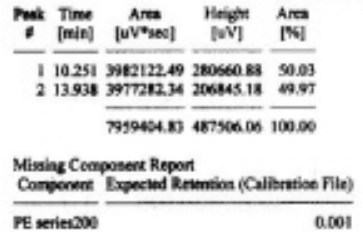

Page 14

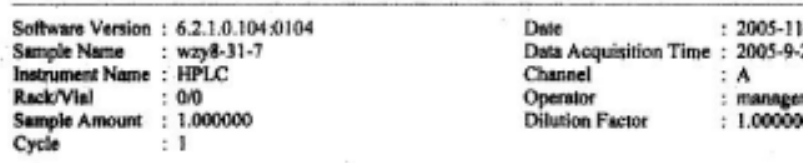

Result File : D:LC CwaryiPh-CH(NH-Ph-4-Cl)-CH3050929-reh-31-7.nt

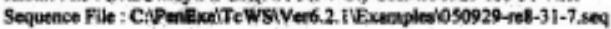

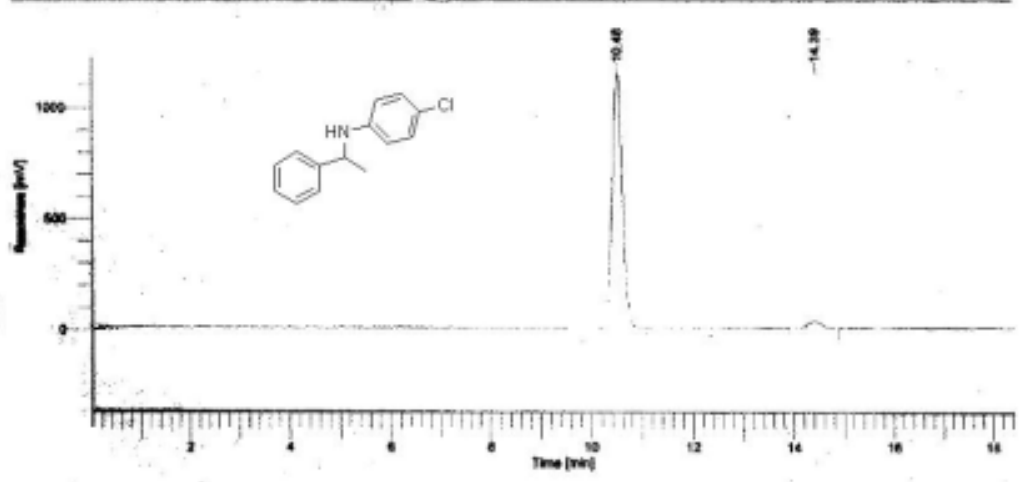

\section{DEFAULT REPORT}

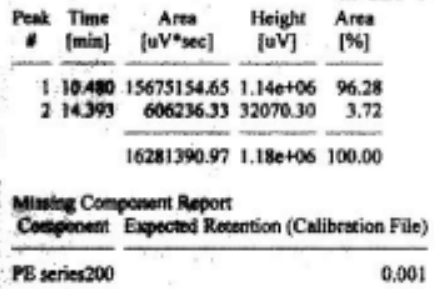


The HPLC spectra of $\mathbf{6 n}$

- Version : 6.2.1.0.104:0104

Name : wrys-40-1

ent Name : HPLC

ial $: 0.0$

Amount $: 1000000$ : 1
Date : 2005-10-12 12:14:45

Drats Acquisition Time : 2005-10-12 11:52:37

Channel : A

: manager

File : DNCCwryph-CH(NH-Ph-4-Br)-CH3 $051012-8-40-1 \times x-15$

oe File: C:PenExeiTeWSiVer6.2.11Exampiesi0s1012-8-40-1xx.seq

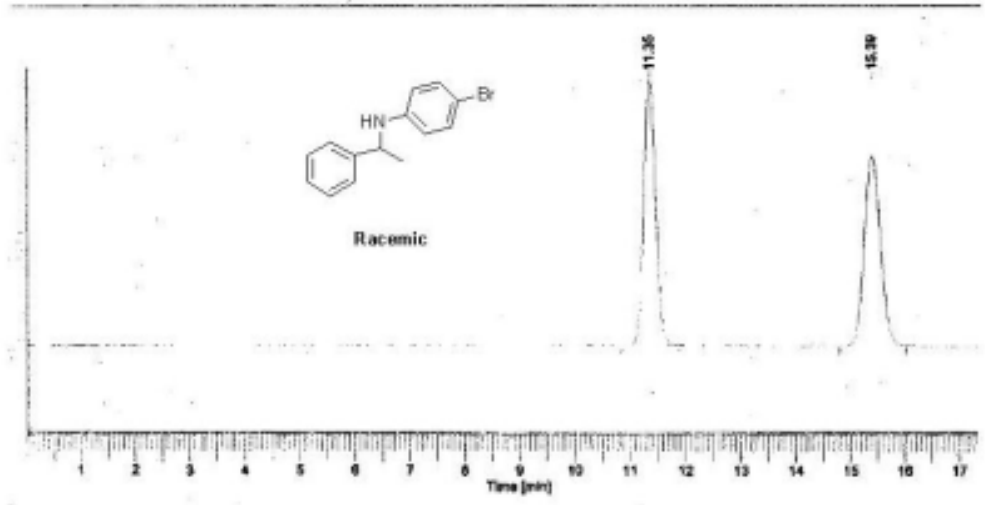

DEFAULT REPORT

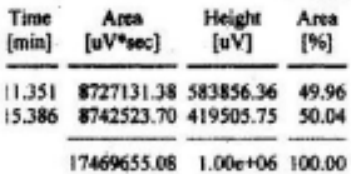

8 Component Report

oneat Expectod Retention (Calibration File)

iex200 0.001

\begin{tabular}{|c|c|c|c|}
\hline $\begin{array}{l}\text { Software Version } \\
\text { Sample Name } \\
\text { Instrument Narte } \\
\text { Rack/Vial } \\
\text { Sample Amoount } \\
\text { Cycle }\end{array}$ & $\begin{array}{l}\text { : } 2.1 .0 .104: 0104 \\
\text { wzyk-41-1 } \\
\text { HPLC } \\
090 \\
1.000000 \\
: 1\end{array}$ & $\begin{array}{l}\text { Date } \\
\text { Dana Acquisitioe Time } \\
\text { Channel } \\
\text { Operanor } \\
\text { Dilution Factor }\end{array}$ & $\begin{array}{l}: 2005-11-215: 33: 57 \\
: 2005-10-1212: 17: 24 \\
: \text { A } \\
: \text { mansor } \\
: 1.000000\end{array}$ \\
\hline
\end{tabular}

Cycle

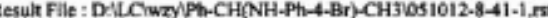

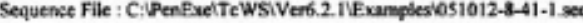

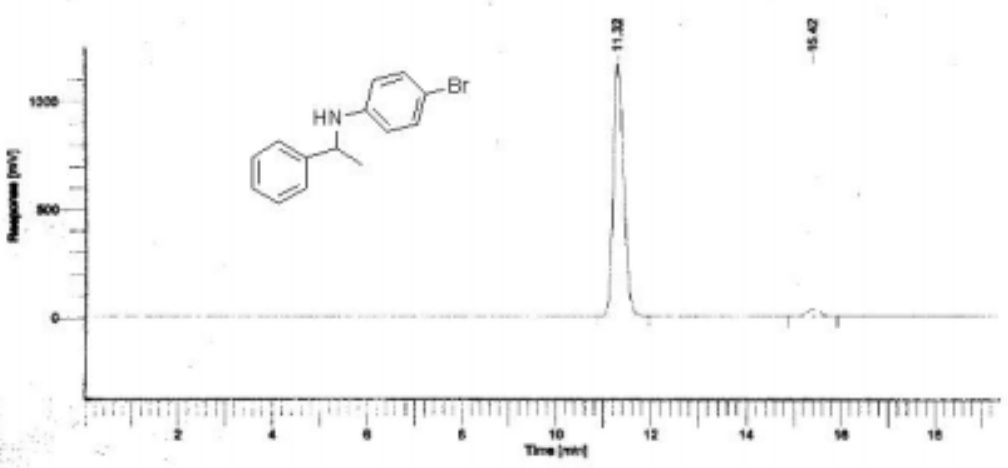

DEFAULT REPORT

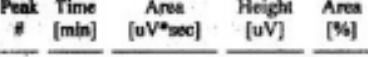

$$
\begin{aligned}
& \overline{18277944.55} \overline{1.20 e+06} \overline{100.00}
\end{aligned}
$$

Missing Component Report

Componeser Expected Retention (Calithration Flle)

PE series200

0.001 
The HPLC spectra of $\mathbf{6 0}$
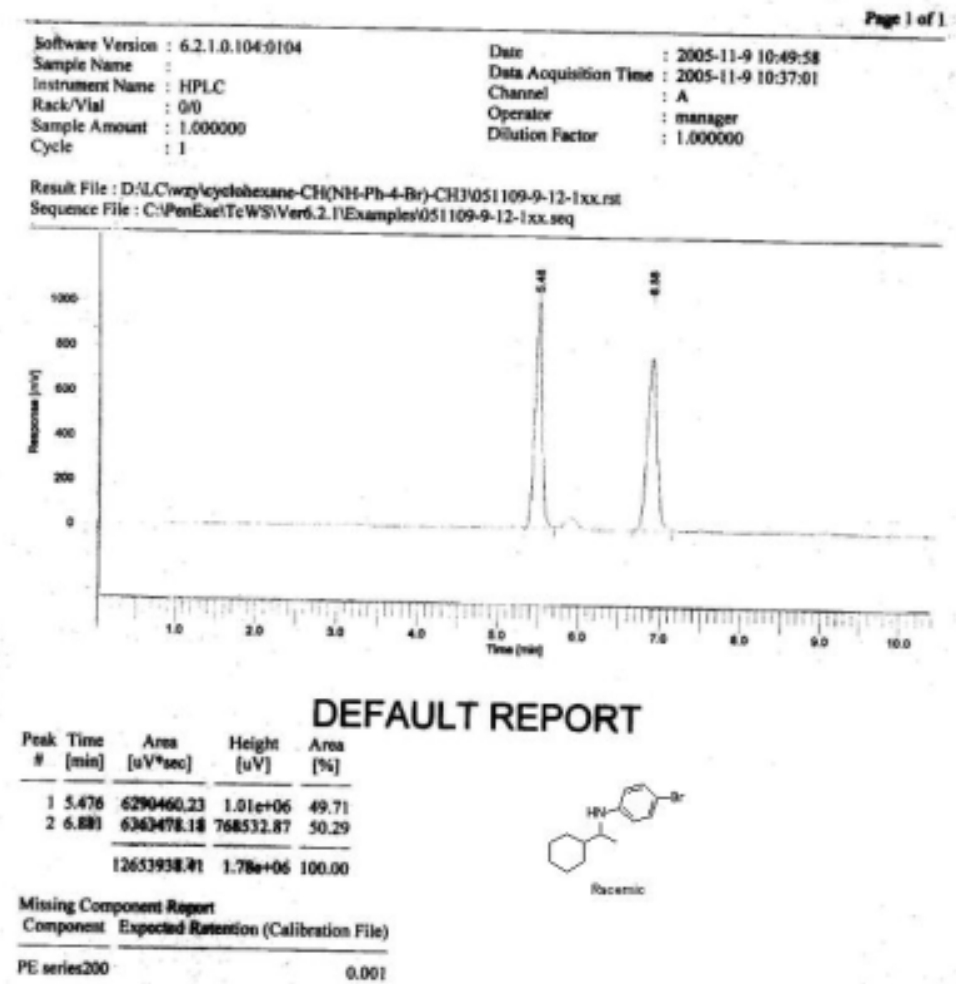

Software Version : 6.2.1.0.104:0104 Sample Name : wzy9-11-1

Instrument Name : HPLC

$\begin{array}{l:l}\text { RackVial } & : 0 / 0 \\ \text { Sample Amount } & 1.000000\end{array}$

Cycle

$: 1$

0.001

Result File : D:LClwzylcyclohexane-CH(NH-Ph-4-Br)-CH31051 109-9-11-1.rst

Sequence File : C:PenExelTcWSIVer6.2.IVxamplest051109-9-11-1.seq

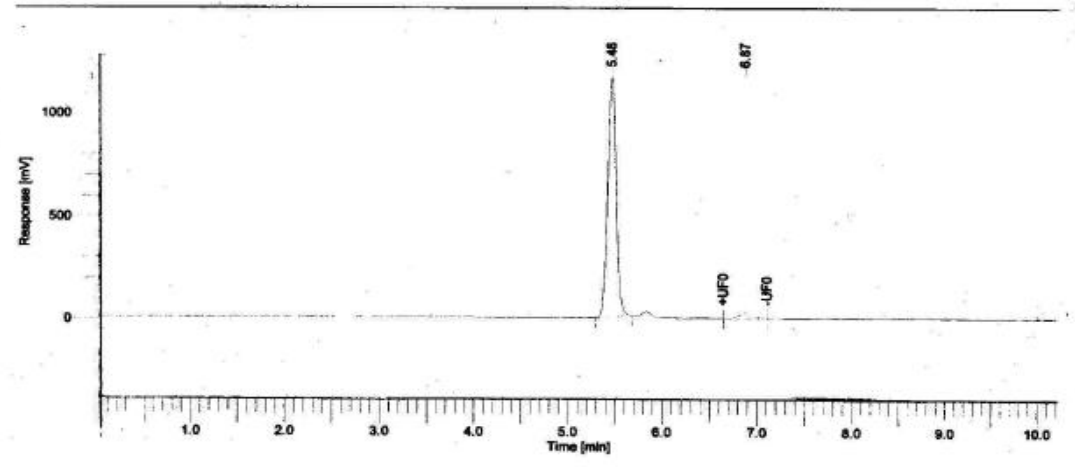

\section{DEFAULT REPORT}

Peak Time
\# [min]
$\left[\mathrm{uV}^{*} \mathrm{sec}\right]$ $\begin{gathered}\text { Height } \\ {[\mathrm{uV}]}\end{gathered} \quad \begin{gathered}\text { Area } \\ {[\%]}\end{gathered}$

$15.4597321936 .68 \frac{1.17 \mathrm{e}+06}{97.31}$

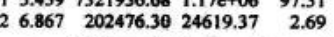

$\overline{7524412.98} \overline{1.196+06} \overline{100.00}$

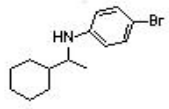

Missing Component Report

Component Expected Retention (Calibration File)

PE series200

0.001 
The HPLC spectra of $\mathbf{6 p}$

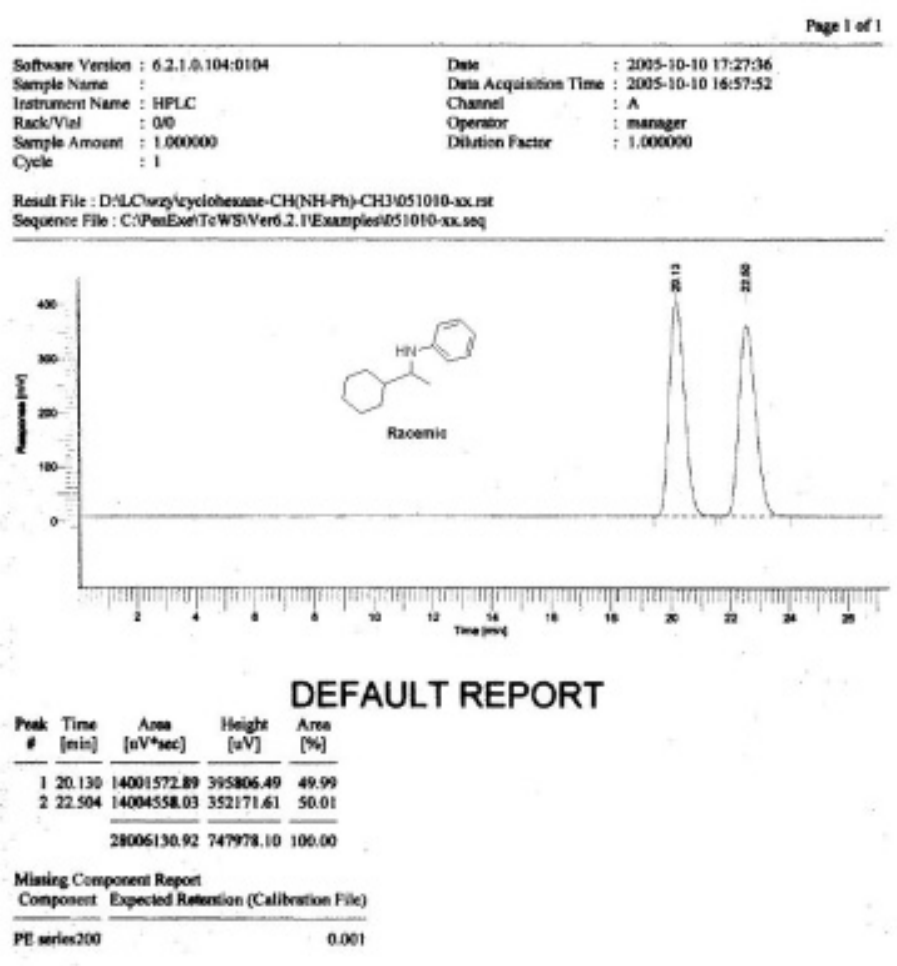

\begin{tabular}{|c|c|c|c|c|}
\hline $\begin{array}{l}\text { Sodtware Version } \\
\text { Sample Name } \\
\text { Instrument Name } \\
\text { Rack/Vlal } \\
\text { Sample Amount } \\
\text { Cycle }\end{array}$ & $\begin{array}{l}=6.2 .1 .0 .104: 0104 \\
\text { wagt-31-3 } \\
\text { HPLC } \\
000 \\
1.000000 \\
1\end{array}$ & 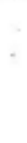 & $\begin{array}{l}\text { Date } \\
\text { Data Acquisition Time } \\
\text { Channel } \\
\text { Operator } \\
\text { Diletiou Factor }\end{array}$ & $\begin{array}{l}: 2005-9-3017: 02: 12 \\
\vdots 2005-9-3016: 32: 35 \\
\vdots \text { A } \\
\vdots \text { Inanager } \\
\vdots: 1.000000\end{array}$ \\
\hline
\end{tabular}

Result File : DiLCwayleyclotexane-CH(NH-Ph)-CH3050930-8-31-3.ns Sequence File : C.PenExeiTcWSIVero.2. ILexamples 1050930-8-31-3.seq

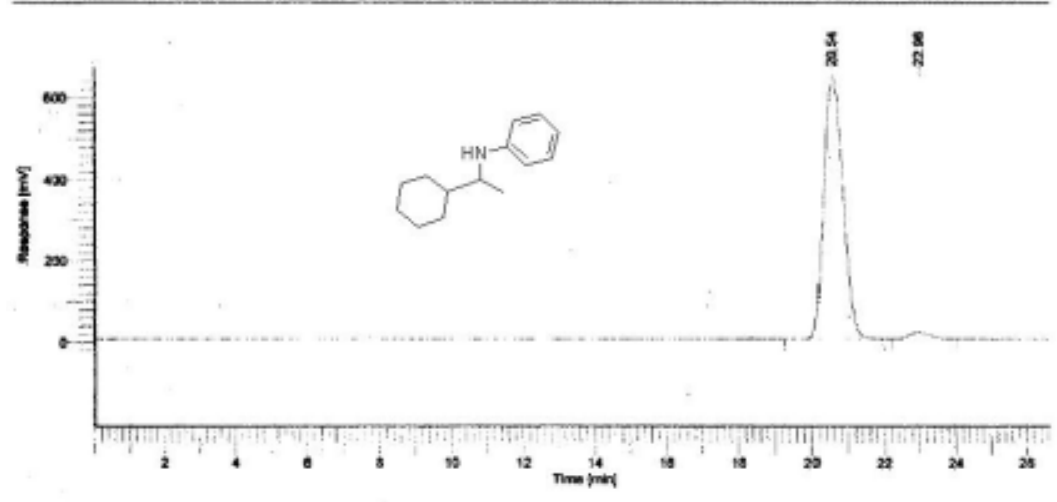

\section{DEFAULT REPORT}

\begin{tabular}{|c|c|c|c|c|}
\hline Peak & $\underset{\text { Time }}{\text { Tin] }}$ & 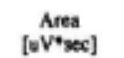 & $\begin{array}{l}\text { Heighin } \\
{[\mathrm{aV}]}\end{array}$ & $\begin{array}{l}\text { Ares } \\
{[\%]}\end{array}$ \\
\hline & $\begin{array}{l}20.543 \\
22.959\end{array}$ & $\begin{array}{r}23137989.07 \\
636412.24\end{array}$ & $\begin{array}{r}639204.51 \\
15824.46\end{array}$ & $\begin{array}{r}97.32 \\
2.68\end{array}$ \\
\hline
\end{tabular}

Missing Componest Report

Componens Expectod Retention (Calibration File)

PE serise 200

0.001 
The HPLC spectra of $\mathbf{6 q}$
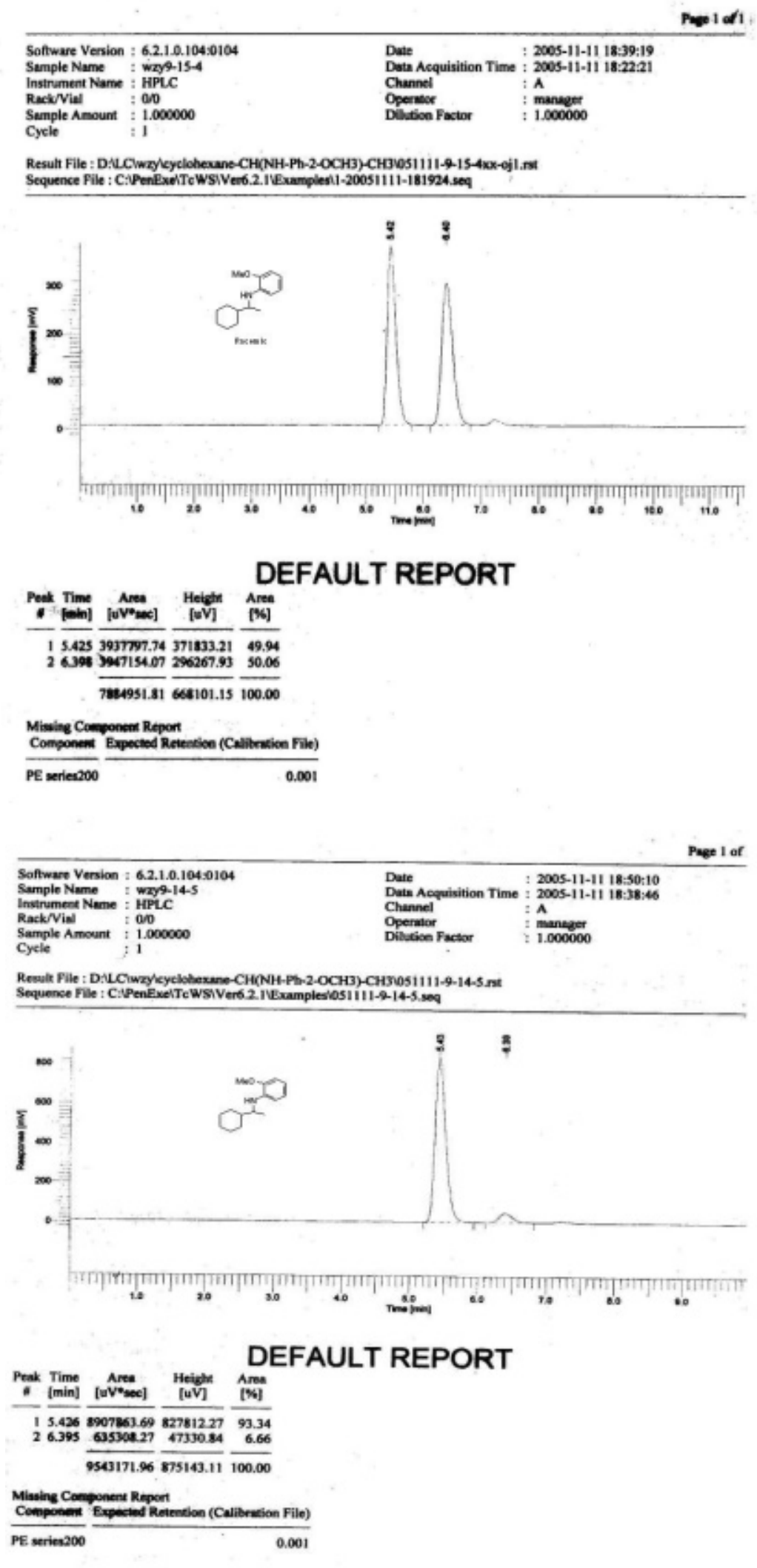
The HPLC spectra of $\mathbf{6 s}$

Page 1 of 1

Software Version : $6.21 .0 .104: 0104$

Sample Nane

Instrument Name : HPLC

Rack/Vial

: : 010

Cycle

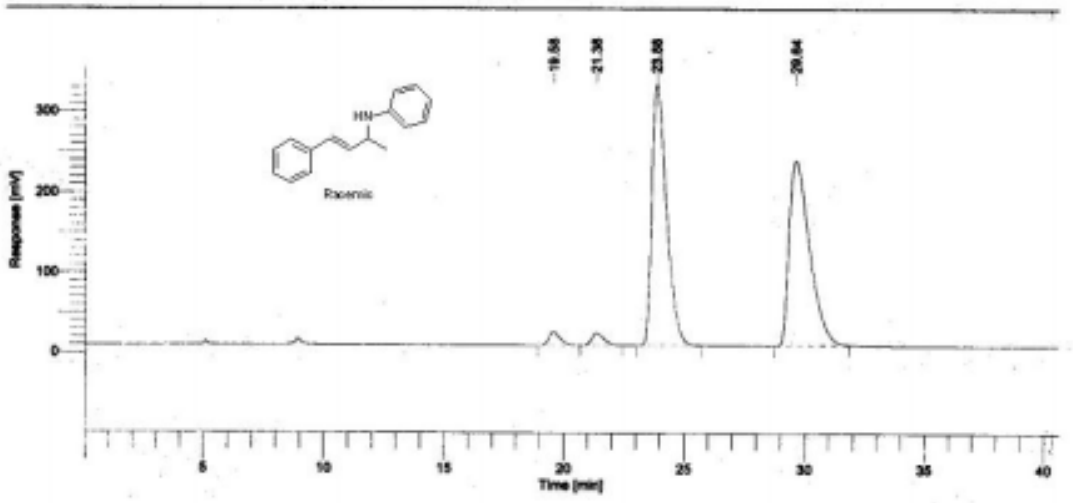

\section{DEFAULT REPORT}

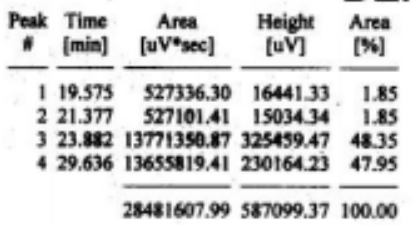

$\begin{array}{lll}\text { Date } & \text { 2005-12-12 16:40:32 } \\ \text { Duta Acquisition Time : } 2005-12-12 \text { 15:57:40 } & \text { : A }\end{array}$

Channel : A

$\begin{array}{ll}\text { Operator } & : \text { manteger } \\ \text { Dilution Factor } & : 1.000000\end{array}$

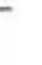

-

